\title{
Characterizing maser polarization: effects of saturation, anisotropic pumping, and hyperfine structure
}

\author{
Boy Lankhaar and Wouter Vlemmings
}

\begin{abstract}
Department of Space, Earth and Environment, Chalmers University of Technology, Onsala Space Observatory, 43992 Onsala, Sweden e-mail: lankhaar@chalmers.se
\end{abstract}

Received 15 January 2019 / Accepted 6 May 2019

\begin{abstract}
Context. The polarization of masers contains information on the magnetic field strength and direction of the regions they occur in. Many maser polarization observations have been performed over the last 30 years. However, versatile maser polarization models that can aide in the interpretation of these observations are not available.

Aims. We developed a program suite that can compute the polarization by a magnetic field of any non-paramagnetic maser species at arbitrarily high maser saturation. Furthermore, we investigated the polarization of masers by non-Zeeman polarizing effects. We present a general interpretive structure for maser polarization observations.

Methods. We expanded existing maser polarization theories of non-paramagnetic molecules and incorporated them in a numerical modeling program suite.

Results. We present a modeling program called CHAracterizes Maser Polarization (CHAMP) that can examine the polarization of masers of arbitrarily high maser saturation and high angular momentum. Hyperfine multiplicity of the maser-transition can also be incorporated. The user is able to investigate non-Zeeman polarizing mechanisms such as anisotropic pumping and polarized incident seed radiation. We present an analysis of the polarization of $v=1 \mathrm{SiO}$ masers and the $22 \mathrm{GHz}$ water maser. We comment on the underlying polarization mechanisms, and also investigate non-Zeeman effects.

Conclusions. We identify the regimes where different polarizing mechanisms will be dominant and present the polarization characteristics of the $\mathrm{SiO}$ and water masers. From the results of our calculations, we identify markers to recognize alternative polarization mechanisms. We show that comparing randomly generated linear versus circular polarization $\left(p_{\mathrm{L}}-p_{\mathrm{V}}\right)$ scatter-plots at fixed magnetic field strength to the observationally obtained $p_{\mathrm{L}}-p_{\mathrm{V}}$ scatter can be a promising method of ascertaining the average magnetic field strength of a large number of masers.
\end{abstract}

Key words. methods: numerical - masers - stars: magnetic field - polarization

\section{Introduction}

Observation of the polarized emission from masers is an established method of obtaining information on the magnetic field in the maser region. Linear polarization reveals the projected magnetic field direction, and circular polarization reveals information on the magnetic field strength. Maser polarization observations have been performed for OH (e.g., Baudry \& Diamond 1998; Fish \& Reid 2006), $\mathrm{H}_{2} \mathrm{O}$ (e.g., Vlemmings et al. 2006a), SiO (e.g., Kemball \& Diamond 1997; Kemball et al. 2009; Herpin et al. 2006), and methanol (e.g., Vlemmings 2008; Vlemmings et al. 2011a; Lankhaar et al. 2018). These observations have indicated, among other things, an ordered magnetic field around asymptotic giant branch (AGB) stars, such as TX Cam (Kemball \& Diamond 1997); a magnetically collimated jet from an evolved star (Vlemmings et al. 2006b); the first extragalactic Zeeman-effect detection in (ultra)luminous infrared galaxies (Robishaw et al. 2008); and the magnetically regulated infall of mass on a massive protostellar disk (Vlemmings et al. 2010).

The analysis of maser-polarization observations is often based on the theories of Goldreich et al. (1973; GKK73), which are derived analytically for masers under the limiting conditions of (i) strong saturation, where the rate of stimulated emission, $R$, is significantly higher than the isotropic decay rate, $\Gamma(R \gg \Gamma)$; (ii) very strong magnetic fields, where the magnetic precession rate $g \Omega$ is significantly higher than $R(g \Omega \gg R)$; and (iii) high thermal widths, where the thermal broadening, $\Delta \omega$ in frequency units, is significantly higher than $g \Omega(\Delta \omega \gg g \Omega)$. These requirements are seldom fulfilled and numerical approaches need to be invoked to ascertain the maser polarization characteristics at intermediary conditions. Well-known numerical approaches to characterizing maser-polarization have been presented by Deguchi \& Watson (1990; D\&W90) and Gray \& Field (1995; G\&F95). The latter models are aimed at the polarization of masers arising from paramagnetic molecules like $\mathrm{OH}$, but can be generalized to masers from a non-paramagnetic species (Gray 2012). Even though the G\&F95 and the D\&W90 models have been shown to be isomorphic (Gray 2003), they have made different assumptions in their formulation. For instance, the direct time-dependence of the population $\left(\rho_{a a}\right.$ and $\left.\rho_{b b}\right)$, coupling elements $\left(\rho_{a b}\right)$, and the electric field elements have been integrated out in the D\&W90 models. This was shown by Trung (2009) to have no impact on the simulation results. Instead, the G\&F95 models do not take into account the off-diagonal elements of the state-populations $\left(\rho_{a a^{\prime}}, a \neq a^{\prime}\right)$. Especially in the regions where magnetic field interactions become comparable to the rates of stimulated emission $(g \Omega \sim R$ ), or when accounting for non-Zeeman effects such as anisotropic pumping of the maser or partially polarized incident radiation, this approximation is not valid.

The D\&W90 models have been applied in a number of incarnations: 
(i) In Nedoluha \& Watson (1990; N\&W90), the maser polarization model of D\&W90 is applied for one frequency. Circular polarization cannot be computed. Linear polarization can be computed in the Stokes $U$ and $Q$ parameters. It is possible to introduce anisotropic pumping. Only one hyperfine subtransition can be accounted for. Nedoluha \& Watson (1990) report that simulations can be made at up to $J=3-2$ transitions. Convergence issues arise for higher angular momentum transitions.

(ii) In Nedoluha \& Watson (1992; N\&W92), the maser polarization model of D\&W90 is applied under the limiting condition of $g \Omega \gg R$. Therefore, the Stokes $U$ component of the radiation can be ignored, and only diagonal elements of the density-population matrices need to be regarded. It is in this respect that this variant of the D\&W90 models is similar to the G\&F95 models. Circular polarization and linear polarization can be computed, but the polarization angle can only be $\chi=0^{\circ}$ or $\chi=90^{\circ}$. Multiple hyperfine sub-transition can be included.

(iii) In Nedoluha \& Watson (1994; N\&W94), we find the most extensive variant of the D\&W90 models. The N\&W94 models account for off-diagonal elements in the populationdensities, the Stokes $U$ component of the radiation field, and multiple frequency bins along the maser-line. Anisotropic pumping can be introduced, but multiple hyperfine subtransitions cannot be included. Because of the computational costs of this approach, N\&W94 only give results for the $J=1-0$ transition.

Only the qualitative results of these approaches are available. In this paper, we present a program that we call $\mathrm{CHAMP}^{1}$ (CHAracterizing Maser Polarization) that simulates the propagation of maser radiation through a medium permeated by a magnetic field. The user is able to adopt the three approaches of N\&W90, N\&W92, and N\&W94. We reproduced these models, and made two significant improvements: the transition of arbitrary angular momentum can be simulated, and the N\&W94 formalism has been expanded to include multiple (and high$F$ ) hyperfine transitions. These improvements are vital when analyzing the polarization of high-frequency masers that have become more relevant in the era of ALMA and its full polarization capabilities (see, e.g., Pérez-Sánchez \& Vlemmings 2013).

As a way of outlining the capabilities of CHAMP, we perform a range of simulations of the non-paramagnetic maser species $\mathrm{SiO}$ and $\mathrm{H}_{2} \mathrm{O}$, and comment on their relation to simplified methods of analysis performed in the past. We focus on a range of $\mathrm{SiO} v=1, J-(J-1)$ maser transitions, and the $22 \mathrm{GHz}$ water maser transition. We present simulations of non-Zeeman polarizing effects, like anisotropy in the maser pumping and polarized seed radiation. We leave maser polarization simulations and analysis of methanol masers, including its complex hyperfine structure (Lankhaar et al. 2016), for a later publication. It is possible to investigate the polarization of any non-paramagnetic maser (e.g., formaldehyde) with CHAMP. The paramagnetic $\mathrm{OH}$ masers can also be investigated with these models, but this would be an unnecessary complication of the maser polarization theory, because simplifications from the complete spectral decoupling of the magnetic sub-transitions are not used.

This paper is organized as follows. In Sect. 2, we recall the theory of maser-radiation put forth by GKK73 and D\&W90, and expand their work by considering multiple hyperfine transitions

\footnotetext{
1 The source code of CHAMP and a number of standard input files are available on GitHub at https://github. com/blankhaar/CHAMP.
}

within a certain rotational maser line. In Sect. 3, we present the three numerical approaches based on N\&W90, N\&W92, and $\mathrm{N} \& \mathrm{~W} 94$, to solve the polarized maser-propagation simulations. We pay extra attention in this section to the improvements made that dealt with previous convergence issues. In Sect. 4 we apply our models to simulate the polarization of $\mathrm{SiO}$ and water masers. In Sect. 5 the results are evaluated by outlining distinguishable polarizing mechanisms, along with an evaluation of some of the existing maser polarization literature. We conclude with a summary of the results in Sect. 6.

\section{Theory}

In the following, we give a general derivation of the polarization of maser radiation through a magnetically aligned medium. First, we consider the effect of an anisotropic radiation field on a magnetically aligned maser medium and the subsequent radiative feedback through the processes of stimulated emission and absorption. Thereafter, we turn to a brief discussion of anisotropic pumping.

\subsection{Maser polarization by a magnetic field}

The theory presented here is based on GKK73, and the extension for numerical modeling (Western \& Watson 1984; Deguchi \& Watson 1990; Nedoluha \& Watson 1994). We extend these formalisms by considering multiple hyperfine transitions that lie close to each other in frequency. Often, it is a rotational transition that is masing, and we consider only the states relevant to this transition, namely the hyperfine manifold and magnetic substates. Interactions of these states with other molecular states (collisionally or radiatively) are absorbed into the phenomenological pumping and decay term. Because the maser-molecules are permeated by a magnetic field, the degeneracy of the magnetic substates is lifted. The consequential spectral decoupling of the photon-transitions with different helicity will cause a polarization of the radiation. This means that we have to consider all the magnetic substates of the maser-transitions, as well as all the modes of polarization in the radiation.

We have not yet mentioned the analytical maser polarization theory by Elitzur (see Elitzur 1991, 1993, 1995, 1998). In this elegant formalism, the no-divergence requirement of the electric component of the radiation field is shown to put constraints on the phases of the propagated electric field polarizations. These phase-relations yield the polarization solutions of GKK73; however, they are general to any degree of saturation. This result is very different from the other theories of maser polarization, including the one we present here. The idealized presuppositions of the Elitzur models, such as the equal populations of magnetic substates throughout propagation, are not reproduced by the D\&W90 and the CHAMP models, while the radiation field is always subject to the constraint of no-divergence. We work within the D\&W90 formalism because of mutual confirmation between G\&F95 and D\&W90 on multiple levels of analysis: the isomorphism of the theories of the D\&W90 and G\&F95 models (Gray 2003; Trung 2009), their reproduction of the earlier derived theories of GKK73, and their strong resemblance to the non-maser radiative transfer models of Degl'Innocenti \& Landolfi (2006).

Setting up the theory of maser radiation propagation can be divided into two parts. On the one hand, we present a model of the occupation of the molecular state-populations under the influence of a polarized radiation field, and on the other hand, 
we present a model of the propagation of the radiation field that is dependent on the state-populations.

\subsubsection{Evolution of the density operator}

Let us consider a maser transition between two (torsion)rotational states. The maser medium is permeated by a magnetic field and the two states are coupled by the radiation field. Before considering the interaction of the radiation field and the two (torsion-)rotation states, we pay special attention to hyperfine splitting of the line. When the molecules total nuclear spin, $I$, is nonzero, both (torsion-)rotational levels participating in the maser-transition are split up further by hyperfine interactions in an ensemble of $n_{F}=2 I+1$ hyperfine states,

$F_{1}^{(1)}, F_{2}^{(1)}, \cdots F_{n_{F}}^{(1)}, \quad F_{1}^{(2)}, F_{2}^{(2)}, \cdots F_{n_{F}}^{(2)}$,

for the upper and the lower level. As a consequence the single maser transition splits up in a manifold of hyperfine transitions, where any transition $F_{i}^{(1)} \rightarrow F_{j}^{(2)}$ is allowed as long as the selection rule $\Delta F=0, \pm 1$ is fulfilled. The hyperfine splitting thus results in a manifold of hyperfine states, where each upper state is radiatively coupled to multiple other lower states.

However, it turns out that each upper hyperfine level is radiatively coupled most strongly to only one lower hyperfine level, dominating other transitions by over an order of magnitude. By virtue of this, we can simplify our problem by decomposing the maser transition into their strongest transitions, $F_{i}^{(1)} \rightarrow F_{i}^{(2)}$, and neglect all other couplings. In this way we are left with $n_{F}$ systems, all independently interacting with the same radiation field.

The Hamiltonian of the $i^{\prime}$ th transition is

$\hat{H}_{i}=\left(\begin{array}{ll}\hat{H}_{i}^{(1)} & \hat{V}_{i}^{(12)} \\ \hat{V}_{i}^{(21)} & \hat{H}_{i}^{(2)}\end{array}\right)$,

where the elements of the diagonal matrix elements are defined in the frame where the magnetic field is along the $z$-axis, and are

$\left\langle F_{i}^{(1)} m_{F}\left|\hat{H}_{i}^{(1)}\right| F_{i}^{(1)} m_{F}\right\rangle=E_{i}^{(1)}+g \Omega_{i}^{(1)} m_{F}$,

$\left\langle F_{i}^{(2)} m_{F}\left|\hat{H}_{i}^{(2)}\right| F_{i}^{(2)} m_{F}\right\rangle=E_{i}^{(2)}+g \Omega_{i}^{(2)} m_{F}$,

where $E_{i}^{(1,2)}$ are the hyperfine energies of the upper and lower level, and $g \Omega_{i}^{(1,2)}$ their respective Zeeman splittings. The coupling elements are

$\hat{V}_{i}^{(12)}=-\hat{\boldsymbol{d}} \cdot \boldsymbol{E}$,

where $\hat{\boldsymbol{d}}$ is the dipole operator, and $\boldsymbol{E}$ is the electric field. With this decomposition, we can formulate the evolution equation for the states for the $n_{F}$ independent systems, following the Liouville-von Neumann equation,

$\dot{\hat{\rho}}_{i}=-\frac{i}{\hbar}\left[\hat{H}_{i}, \hat{\rho}_{i}\right]+\hat{\Lambda}_{i}-\hat{\Gamma}_{i} \hat{\rho}_{i}$,

where we take into account the excitation of both levels by including a phenomenological term for the pumping of the maser, $\hat{\Lambda}_{i}$, and the decay of the states by $\hat{\Gamma}_{i}$. Just as in the Hamiltonian in Eq. (1), we can express the density-operator in its parts,

$\hat{\rho}_{i}=\left(\begin{array}{cc}\hat{\rho}_{i}^{(1)} & \hat{\rho}_{i}^{(12)} \\ \hat{\rho}_{i}^{(21)} & \hat{\rho}_{i}^{(2)}\end{array}\right)$, so that the evolution of the decomposed density operators is

$$
\begin{aligned}
\dot{\hat{\rho}}_{i}^{(1)}= & -\frac{i}{\hbar}\left(\left[\hat{H}_{i}^{(1)}, \hat{\rho}_{i}^{(1)}\right]+\hat{V}_{i}^{(12)} \hat{\rho}_{i}^{(21)}-\hat{\rho}_{i}^{(12)} \hat{V}_{i}^{(21)}\right) \\
& -\hat{\Gamma}_{i}^{(1)} \hat{\rho}_{i}^{(1)}+\hat{\Lambda}_{i}^{(1)}, \\
\dot{\hat{\rho}}_{i}^{(2)}= & -\frac{i}{\hbar}\left(\left[\hat{H}_{i}^{(2)}, \hat{\rho}_{i}^{(2)}\right]+\hat{V}_{i}^{(21)} \hat{\rho}_{i}^{(12)}-\hat{\rho}_{i}^{(21)} \hat{V}_{i}^{(12)}\right) \\
& -\hat{\Gamma}_{i}^{(2)} \hat{\rho}_{i}^{(2)}+\hat{\Lambda}_{i}^{(2)}, \\
\dot{\hat{\rho}}_{i}^{(12)}= & -\frac{i}{\hbar}\left(\hat{H}_{i}^{(1)} \hat{\rho}_{i}^{(12)}-\hat{\rho}_{i}^{(12)} \hat{H}_{i}^{(2)}+\hat{V}_{i}^{(12)} \hat{\rho}_{i}^{(2)}-\hat{\rho}_{i}^{(1)} \hat{V}_{i}^{(12)}\right) \\
& -\hat{\Gamma}_{i}^{(1)} \hat{\rho}_{i}^{(12)} .
\end{aligned}
$$

In D\&W90 it is shown how to integrate out the timedependence of the off-diagonal elements of Eq. (6c). The solutions of these integrations, are subsequently inserted into the population-equations of Eqs. (6a) and (6b). We assume a steady state: $\dot{\hat{\rho}}_{i}^{(1)}=\dot{\hat{\rho}}_{i}^{(2)}=0$. After somewhat involved rearrangements that are analogous to those used in D\&W90, we find the expressions for the upper state-populations

$$
\begin{aligned}
& 0=-\left(\Gamma_{i}+i \omega_{a_{i} a_{i}^{\prime}}\right) \rho_{a_{i} a_{i}^{\prime}}(v)+\phi(v) \lambda_{a_{i} a_{i}^{\prime}}
\end{aligned}
$$

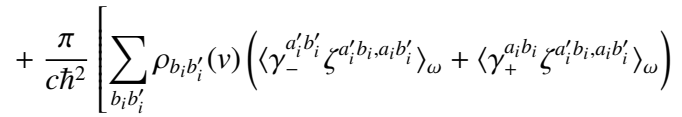

$$
\begin{aligned}
& \left.-\sum_{b_{i} a_{i}^{\prime \prime}} \rho_{a_{i}^{\prime \prime} a_{i}^{\prime}}(v)\left\langle\gamma_{-}^{a_{i}^{\prime} b_{i}}\left(\zeta^{a_{i} b_{i}, a_{i}^{\prime \prime} b_{i}}\right)^{*}\right\rangle_{\omega}-\sum_{b_{i} a_{i}^{\prime \prime}} \rho_{a_{i} a_{i}^{\prime \prime}}(v)\left\langle\gamma_{+}^{a_{i} b_{i}}\left(\zeta^{a_{i}^{\prime} b_{i}, a_{i}^{\prime \prime} b_{i}}\right)^{*}\right\rangle_{\omega}\right] \text {, }
\end{aligned}
$$

where $a_{i}$ and $b_{i}$ are the indices for the magnetic substates of the upper and lower levels, and the energy difference between magnetic substates are represented by $\hbar \omega_{a a^{\prime}}=E_{a}-E_{a^{\prime}}$. Elements of the pumping operator are represented as $\Lambda_{a a^{\prime}}=\phi(v) \lambda_{a a^{\prime}}$, where $\phi(v)$ stands for the Maxwell-Boltzmann distribution. Furthermore, we use the simplified notations

$\zeta^{i j, k l}=I(\omega) \delta_{I}^{i j, k l}-Q(\omega) \delta_{Q}^{i j, k l}-i U(\omega) \delta_{U}^{i j, k l}+V(\omega) \delta_{V}^{i j, k l}$,

and $(I, Q, U, V)$ are the Stokes parameters as defined in D\&W90. The delta-operators are related to the dipole elements by

$\delta_{I}^{i j, k l}=\left(d_{+}^{i j}\right)^{*} d_{+}^{k l}+\left(d_{-}^{i j}\right)^{*} d_{-}^{k l}$,

$\delta_{Q}^{i j, k l}=\left(d_{+}^{i j}\right)^{*} d_{-}^{k l}+\left(d_{-}^{i j}\right)^{*} d_{+}^{k l}$,

$\delta_{U}^{i j, k l}=\left(d_{+}^{i j}\right)^{*} d_{-}^{k l}-\left(d_{-}^{i j}\right)^{*} d_{+}^{k l}$,

$\delta_{V}^{i j, k l}=\left(d_{+}^{i j}\right)^{*} d_{+}^{k l}-\left(d_{-}^{i j}\right)^{*} d_{-}^{k l}$,

with explicit elements (D\&W90)

$d_{ \pm}^{a b}= \pm d_{M=1}^{a b} \frac{1 \pm \cos \theta}{2}+i d_{M=0}^{a b} \frac{\sin \theta}{\sqrt{2}} \mp d_{M=-1}^{a b} \frac{1 \mp \cos \theta}{2}$

where $\theta$ is the angle between the magnetic field and propagation directions. We also use a simplified notation for the integral

$\left\langle\gamma_{ \pm}^{i j} \zeta^{k l, m n}\right\rangle_{\omega}=\int \mathrm{d} \omega \gamma_{ \pm}^{i j}(\omega, v) \zeta^{k l, m n}(\omega)$

with

$\gamma_{ \pm}^{a_{i} b_{i}}=\frac{1}{\Gamma_{i} \pm i\left[\omega_{a_{i} b_{i}}-\omega\left(1-\frac{v}{c}\right)\right]}$

The lower state-populations follow from a similar derivation. The population equations for the upper- and lower-level of the 
hyperfine transition $F_{i}^{(1)} \rightarrow F_{i}^{(2)}$, are mutually dependent, but do not depend directly on other hyperfine transitions within our approximation, as was motivated at the beginning of this section. We thus have a set of $n_{F}$-independent population equations for the hyperfine substates of the rotational transition under investigation.

\subsubsection{Evolution of the radiation field}

The evolution of polarized radiation has been derived elsewhere (e.g., Goldreich et al. 1973; Deguchi \& Watson 1990; Degl'Innocenti \& Landolfi 2006). We re-iterate the expressions here, while also taking into account the feed of multiple closelying hyperfine transitions to the radiation field. By using the well-known relation between the propagation of the electric field and the polarization of the medium, it is possible to find the connection between the radiative propagation and the molecular states by expressing the medium polarization in terms of the expectation value of the molecular dipole moment of the ensemble. After expressing the polarization in terms of the molecular states, while maintaining attentive to polarization, we arrive at the propagation relation for the Stokes parameters (Goldreich et al. 1973; Nedoluha \& Watson 1992)

$$
\frac{d}{\mathrm{~d} s}\left(\begin{array}{c}
I(\omega) \\
Q(\omega) \\
U(\omega) \\
V(\omega)
\end{array}\right)=\left(\begin{array}{cccc}
A(\omega) & B(\omega) & F(\omega) & C(\omega) \\
B(\omega) & A(\omega) & E(\omega) & G(\omega) \\
F(\omega) & -E(\omega) & A(\omega) & D(\omega) \\
C(\omega) & -G(\omega) & -D(\omega) & A(\omega)
\end{array}\right)\left(\begin{array}{c}
I(\omega) \\
Q(\omega) \\
U(\omega) \\
V(\omega)
\end{array}\right)
$$

The expressions for the propagation coefficients are

$$
\begin{aligned}
& A(\omega)=\frac{-\pi \omega}{c \hbar} \sum_{i} \sum_{a_{i} b_{i}} \int \mathrm{d} v\left[\sum_{b_{i}^{\prime}}\left\langle\rho_{b_{i}^{\prime} b_{i}}\left(\gamma_{+}^{a_{i} b_{i}}+\gamma_{-}^{a_{i} b_{i}^{\prime}}\right)\right\rangle \delta_{I}^{a_{i} b_{i}, a_{i} b_{i}^{\prime}}\right. \\
& \left.-\sum_{a_{i}^{\prime}}\left\langle\rho_{a_{i} a_{i}^{\prime}}\left(\gamma_{+}^{a_{i} b_{i}}+\gamma_{-}^{a_{i} b_{i}^{\prime}}\right)\right\rangle \delta_{I}^{a_{i} b_{i}, a_{i}^{\prime} b_{i}}\right] \\
& B(\omega)=\frac{\pi \omega}{c \hbar} \sum_{i} \sum_{a_{i} b_{i}} \int \mathrm{d} v\left[\sum_{b_{i}^{\prime}}\left\langle\rho_{b_{i}^{\prime} b_{i}}\left(\gamma_{+}^{a_{i} b_{i}}+\gamma_{-}^{a_{i} b_{i}^{\prime}}\right)\right\rangle \delta_{Q}^{a_{i} b_{i}, a_{i} b_{i}^{\prime}}\right. \\
& \left.-\sum_{a_{i}^{\prime}}\left\langle\rho_{a_{i} a_{i}^{\prime}}\left(\gamma_{+}^{a_{i} b_{i}}+\gamma_{-}^{a_{i} b_{i}^{\prime}}\right)\right\rangle \delta_{Q}^{a_{i} b_{i}, a_{i}^{\prime} b_{i}}\right] \\
& C(\omega)=\frac{-\pi \omega}{c \hbar} \sum_{i} \sum_{a_{i} b_{i}} \int \mathrm{d} v\left[\sum_{b_{i}^{\prime}}\left\langle\rho_{b_{i}^{\prime} b_{i}}\left(\gamma_{+}^{a_{i} b_{i}}+\gamma_{-}^{a_{i} b_{i}^{\prime}}\right)\right\rangle \delta_{V}^{a_{i} b_{i}, a_{i} b_{i}^{\prime}}\right. \\
& \left.-\sum_{a_{i}^{\prime}}\left\langle\rho_{a_{i} a_{i}^{\prime}}\left(\gamma_{+}^{a_{i} b_{i}}+\gamma_{-}^{a_{i} b_{i}^{\prime}}\right)\right\rangle \delta_{V}^{a_{i} b_{i}, a_{i}^{\prime} b_{i}}\right] \\
& D(\omega)=\frac{-i \pi \omega}{c \hbar} \sum_{i} \sum_{a_{i} b_{i}} \int \mathrm{d} v\left[\sum_{b_{i}^{\prime}}\left\langle\rho_{b_{i}^{\prime} b_{i}}\left(\gamma_{+}^{a_{i} b_{i}}-\gamma_{-}^{a_{i} b_{i}^{\prime}}\right)\right\rangle \delta_{Q}^{a_{i} b_{i}, a_{i} b_{i}^{\prime}}\right. \\
& \left.-\sum_{a_{i}^{\prime}}\left\langle\rho_{a_{i} a_{i}^{\prime}}\left(\gamma_{+}^{a_{i} b_{i}}-\gamma_{-}^{a_{i} b_{i}^{\prime}}\right)\right\rangle \delta_{Q}^{a_{i} b_{i}, a_{i}^{\prime} b_{i}}\right] \\
& E(\omega)=\frac{i \pi \omega}{c \hbar} \sum_{i} \sum_{a_{i} b_{i}} \int \mathrm{d} v\left[\sum_{b_{i}^{\prime}}\left\langle\rho_{b_{i}^{\prime} b_{i}}\left(\gamma_{+}^{a_{i} b_{i}}-\gamma_{-}^{a_{i} b_{i}^{\prime}}\right)\right\rangle \delta_{V}^{a_{i} b_{i}, a_{i} b_{i}^{\prime}}\right. \\
& \left.-\sum_{a_{i}^{\prime}}\left\langle\rho_{a_{i} a_{i}^{\prime}}\left(\gamma_{+}^{a_{i} b_{i}}-\gamma_{-}^{a_{i} b_{i}^{\prime}}\right)\right\rangle \delta_{V}^{a_{i} b_{i}, a_{i}^{\prime} b_{i}}\right]
\end{aligned}
$$

$$
\begin{aligned}
F(\omega)= & \frac{i \pi \omega}{c \hbar} \sum_{i} \sum_{a_{i} b_{i}} \int \mathrm{d} v\left[\sum_{b_{i}^{\prime}}\left\langle\rho_{b_{i}^{\prime} b_{i}}\left(\gamma_{+}^{a_{i} b_{i}}+\gamma_{-}^{a_{i} b_{i}^{\prime}}\right)\right\rangle \delta_{U}^{a_{i} b_{i}, a_{i} b_{i}^{\prime}}\right. \\
& \left.-\sum_{a_{i}^{\prime}}\left\langle\rho_{a_{i} a_{i}^{\prime}}\left(\gamma_{+}^{a_{i} b_{i}}+\gamma_{-}^{a_{i} b_{i}^{\prime}}\right)\right\rangle \delta_{U}^{a_{i} b_{i}, a_{i}^{\prime} b_{i}}\right] \\
G(\omega)= & \frac{-\pi \omega}{c \hbar} \sum_{i} \sum_{a_{i} b_{i}} \int \mathrm{d} v\left[\sum_{b_{i}^{\prime}}\left\langle\rho_{b_{i}^{\prime} b_{i}}\left(\gamma_{+}^{a_{i} b_{i}}+\gamma_{-}^{a_{i} b_{i}^{\prime}}\right)\right\rangle \delta_{U}^{a_{i} b_{i}, a_{i} b_{i}^{\prime}}\right. \\
& \left.-\sum_{a_{i}^{\prime}}\left\langle\rho_{a_{i} a_{i}^{\prime}}\left(\gamma_{+}^{a_{i} b_{i}}-\gamma_{-}^{a_{i} b_{i}^{\prime}}\right)\right\rangle \delta_{U}^{a_{i} b_{i}, a_{i}^{\prime} b_{i}}\right]
\end{aligned}
$$

where the sum $i$ runs over all hyperfine transitions, and $a_{i}$ and $b_{i}$ are the magnetic sublevels of the upper and lower level, respectively, of the $i^{\prime}$ th hyperfine transition. The tight relation between the molecular states and the feed to the radiation field is also reflected in these equations as again, the radiative coupling between the two states is represented by the $\delta$-operators. In Sect. 3 we outline the three approaches to numerically solve Eqs. (7) and (14).

\subsection{Anisotropic pumping}

The pumping-operator is a phenomenological term that, together with the decay-operator, absorbs all the interactions with molecular states that are not participating in the maser-transition. The decay-operator is concerned with the decay of the maser-levels to other states. The pumping-operator encapsulates the collisional and radiative (de-)excitations that will eventually populate our two maser-levels.

Alignment will manifest itself in a molecular state when a (de-)excitation to it has a preferred direction. An example of a directional excitation would be the directional pumping radiation, like the radiation from central stellar object that leads to the $\mathrm{SiO}$ maser (Gray 2012). The introduced alignment in the directionally excited molecular state will be transferred (with some depolarization) from state to state in the cascade to our maser levels. The reflection of this anisotropy in the pumping operator was already formulated by Nedoluha \& Watson (1990) and Western \& Watson $(1983,1984)$, who defined the elements of a the anisotropic pumping operator as

$\Lambda_{m m^{\prime}}=\lambda\left(1+\epsilon\left[\frac{F^{2}+F-1+m^{2}}{(2 F-1)(2 F+3)}-1\right]\right) \delta_{m m^{\prime}}$,

where $\lambda$ is the overall pumping, $F$ is the total angular momentum of the associated state, $m$ is the magnetic quantum number, $\delta_{m m^{\prime}}$ is the Kronecker-delta, and $\epsilon$ is the degree of anisotropy in the pumping. In Eq. (15) we assume the direction of the anisotropic pumping to be along the magnetic field direction. If the pumping direction has a different orientation with respect to the magnetic field, the pumping matrix can be obtained by the simple rotation

$\boldsymbol{\Lambda}^{\prime}=\boldsymbol{D}^{\dagger}\left(\alpha^{\prime} \beta^{\prime} \gamma^{\prime}\right) \boldsymbol{\Lambda} \boldsymbol{D}\left(\alpha^{\prime} \beta^{\prime} \gamma^{\prime}\right)$

over the Euler-angles $\left(\alpha^{\prime} \beta^{\prime} \gamma^{\prime}\right)$ that describe the rotation from the pumping-direction to the magnetic field direction.

The partial alignment of the directionally pumped maser will result in the emittance of partially polarized radiation. The polarization will depend not only on the degree of anisotropy in the pumping, $\epsilon$, but will also be dependent on the pumping efficiency,

$\eta=\frac{\epsilon}{\delta}$ 
where we let $\eta$ be the anisotropy parameter, and

$\delta=2 \frac{\lambda_{\mathrm{u}}-\lambda_{1}}{\lambda_{\mathrm{u}}+\lambda_{1}}$

is the pumping-efficiency, with the overall-pumping of the upper and lower level given by $\lambda_{\mathrm{u}, 1}$. The pumping efficiency was investigated for water masers. Estimation of the mean population inversion $\Delta n$ from high-resolution observations of water masers around AGB stars revealed for most masers $\Delta n \lesssim 0.01$. The most luminous masers had higher degrees of population inversion, up to $\delta \sim 0.1$ (Richards et al. 2011). It is to be expected for the more saturated masers that the population inversion will decrease. Richards et al. (2011) estimated that most masers in the sample, though, were unsaturated. For unsaturated masers, their mean population inversion reflects the pumping efficiency $2 \Delta n \sim \delta$; thus, we estimate $\delta \sim 0.02$. The anisotropy degree $\epsilon$ of anisotropically pumped masers is estimated to be of the same order of magnitude (Nedoluha \& Watson 1990).

\section{Methods}

The maser polarization equations that we have derived in the theory section have to be solved numerically. In the following, we outline three numerical approaches that we adopt in CHAMP. We dedicate extra attention on the method we use to solve the density equations in Sect. 2.2. After an outline of the methods we use in CHAMP, we discuss the set-up of the experiments we perform to present the capabilities of CHAMP in Sect. 3.2.

\subsection{Three numerical approaches}

In this work, we reproduce and extend the numerical approximations reported in N\&W90, N\&W92, and N\&W94. The differences between the approaches can be traced back to different approximations to the integrals in Eqs. (7) and (14):

(i) In N\&W90, integrals are approximated to peak sharply around the maximum of $\gamma_{ \pm}^{i j}$,

$\int \mathrm{d} \omega \gamma_{ \pm}^{i j}(\omega, v) \zeta^{k l, m n}(\omega) \approx \pi \zeta^{k l, m n}\left(\omega_{i j}\right)$

where $\omega_{i j}$ is the transition frequency between levels $i$ and $j$. Similarly, the integration over density matrix elements is

$\int \mathrm{d} v \rho_{i j}(v) \gamma_{ \pm}^{k l}(\omega, v)=\frac{\pi c}{\omega_{0}} \rho_{i j}\left(v_{0}\right)$.

We only account for one frequency and velocity bin in the Stokes parameters and density matrix elements. A consequence of this approximation is that only one hyperfine transition can be included and that circular polarization is not computed. Within this approximation we are left with the following simplified density equations $\left(\rho_{i j}=\rho_{i j}\left(v_{0}\right)\right.$ and $\left.\zeta^{i j, k l}=\zeta^{i j, k l}\left(\omega_{0}\right)\right)$,

$$
\begin{aligned}
0= & -\left(\Gamma+i \omega_{a a^{\prime}}\right) \rho_{a a^{\prime}}+\lambda_{a a^{\prime}}+\frac{2 \pi^{2}}{c \hbar^{2}}\left[\sum_{b b^{\prime}} \rho_{b b^{\prime}} \zeta^{a^{\prime} b, a b^{\prime}}\right. \\
& \left.-\sum_{b a^{\prime \prime}} \rho_{a^{\prime \prime} a^{\prime}}\left(\zeta^{a b, a^{\prime \prime} b}\right)^{*}-\sum_{b a^{\prime \prime}} \rho_{a a^{\prime \prime}}\left(\zeta^{a^{\prime} b, a^{\prime \prime} b}\right)^{*}\right],
\end{aligned}
$$

where we dropped the $i$-indices because we cannot treat a hyperfine manifold in this method. Similarly, the propagation coefficients are

$$
A(\omega)=\frac{-2 \pi^{2}}{\hbar} \sum_{a b}\left[\sum_{b^{\prime}} \rho_{b^{\prime} b} \delta_{I}^{a b, a b^{\prime}}-\sum_{a^{\prime}} \rho_{a a^{\prime}} \delta_{I}^{a b, a^{\prime} b}\right],
$$

$$
\begin{aligned}
& B(\omega)=\frac{2 \pi^{2} \omega}{c} \sum_{a b}\left[\sum_{b^{\prime}} \rho_{b^{\prime} b} \delta_{Q}^{a b, a b^{\prime}}-\sum_{a^{\prime}} \rho_{a a^{\prime}} \delta_{Q}^{a b, a^{\prime} b}\right], \\
& C(\omega)=\frac{-2 \pi^{2} \omega}{c} \sum_{a b}\left[\sum_{b^{\prime}} \rho_{b^{\prime} b} \delta_{V}^{a b, a b^{\prime}}-\sum_{a^{\prime}} \rho_{a a^{\prime}} \delta_{V}^{a b, a^{\prime} b}\right], \\
& F(\omega)=\frac{-2 i \pi^{2}}{\hbar} \sum_{a b}\left[\sum_{b^{\prime}} \rho_{b^{\prime} b} \delta_{U}^{a b, a b^{\prime}}-\sum_{a^{\prime}} \rho_{a a^{\prime}} \delta_{U}^{a b, a^{\prime} b}\right],
\end{aligned}
$$

and $D(\omega)=E(\omega)=G(\omega)=0$.

(ii) N\&W92 assume a strong magnetic field. Thus, from Eq. (7), under the limiting condition $g \Omega \gg R$, it follows that diagonal elements dominate the density-populations and that we can neglect off-diagonal elements. Through this simplification we can assume the Stokes $U$ component of the radiation absent. Integrals are simplified in the following way:

$\int \mathrm{d} \omega \gamma_{ \pm}^{a b}(\omega, v) \zeta^{a^{\prime} b^{\prime}, a^{\prime \prime} b^{\prime \prime}}(\omega) \approx \pi \zeta^{a^{\prime} b^{\prime}, a^{\prime \prime} b^{\prime \prime}}\left(\omega_{a b} /(1-v / c)\right)$

$\int \mathrm{d} v \gamma_{ \pm}^{a b}(\omega, v) \rho_{k k}(v) \approx \frac{\pi c}{\omega_{0}} \rho_{k k}\left(c\left(\omega-\omega_{a b}\right) / \omega\right)$

The populations and $\zeta$ parameters are evaluated for $2 N+1$ channels

$\omega=\left\{\omega_{-N}, \omega_{-N+1}, \cdots, \omega_{0}, \cdots, \omega_{N}\right\}$,

where $\omega_{j}=\omega_{0}+j \Delta \omega$, and $\Delta \omega$ is the width of the frequency channel. The frequency channels are related to the velocity channels as $\boldsymbol{v}=\frac{c_{0}}{\omega_{0}} \boldsymbol{\omega}$, so that $v_{j}=j \Delta v=j \frac{c_{0}}{\omega_{0}} \Delta \omega$. For each channel, the population and $\zeta$ parameters are

$$
\begin{aligned}
& \rho_{k k}\left(c\left(\omega_{j}-\omega_{a b}\right) / \omega\right) \approx \rho_{k k}\left(v_{j}\right) \\
& -\left.\frac{c}{\omega_{0}} \frac{\partial \rho_{k k}}{\partial v}\right|_{v_{j}}\left(m_{a} g \Omega_{1} / 2-m_{b} g \Omega_{2} / 2\right) \\
& \zeta^{a^{\prime} b^{\prime}, a^{\prime \prime} b^{\prime \prime}}\left(\omega_{a b} /\left(1-v_{j} / c\right)\right) \approx \zeta^{a^{\prime} b^{\prime}, a^{\prime \prime} b^{\prime \prime}}\left(\omega_{j}\right) \\
& +\left.\frac{\partial \zeta^{a^{\prime} b^{\prime}, a^{\prime \prime} b^{\prime \prime}}}{\partial \omega}\right|_{\omega_{j}}\left(m_{a} g \Omega_{1} / 2-m_{b} g \Omega_{2} / 2\right),
\end{aligned}
$$

leading to simplified density equations and to simplified propagation coefficients, where $D(\omega)=E(\omega)=F(\omega)=G(\omega)=$ 0 . Thus, within this method, propagation of the Stokes $U$ part of the radiation does not occur and can be left out.

(iii) If we follow $\mathrm{N} \& \mathrm{~W} 94$, we do not make any of the approximations outlined above. Rather, we endeavor to solve the Eqs. (7) and (14) by making a numerical approximation to the integral

$$
\int \mathrm{d} \omega \gamma_{ \pm}^{a b}\left(\omega, v_{j}\right) \zeta^{a^{\prime} b^{\prime}, a^{\prime \prime} b^{\prime \prime}}(\omega)
$$

by dividing $\omega$ and $v$ in $2 N+1$-channels, as we do for the N\&W92 method (see above). The first approximation that we make neglects all contributions to the integral outside of the boundaries $\omega_{ \pm N} \pm \Delta \omega / 2$

$$
\begin{aligned}
\int \mathrm{d} \omega & \gamma_{ \pm}^{a b}\left(\omega, v_{j}\right) \zeta^{a^{\prime} b^{\prime}, a^{\prime \prime} b^{\prime \prime}}(\omega) \\
& \approx \int_{\omega_{-N}-\Delta \omega / 2}^{\omega_{N}+\Delta \omega / 2} \mathrm{~d} \omega \gamma_{ \pm}^{a b}\left(\omega, v_{j}\right) \zeta^{a^{\prime} b^{\prime}, a^{\prime \prime} b^{\prime \prime}}(\omega),
\end{aligned}
$$

which is a good approximation for $\omega_{N} \gg \omega_{\mathrm{D}}\left(\omega_{\mathrm{D}}\right.$ is the Doppler broadening). Then, we divide the integral in their respective channels 


$$
\begin{aligned}
& \int_{\omega_{-N}-\Delta \omega / 2}^{\omega_{N}+\Delta \omega / 2} \mathrm{~d} \omega \gamma_{ \pm}^{a b}\left(\omega, v_{j}\right) \zeta^{a^{\prime} b^{\prime}, a^{\prime \prime} b^{\prime \prime}}(\omega) \\
& \quad=\sum_{i=-N}^{N} \int_{-\Delta \omega / 2}^{\Delta \omega / 2} \mathrm{~d} \omega^{\prime} \gamma_{ \pm}^{a b}\left(\omega_{i}+\omega^{\prime}, v_{j}\right) \zeta^{a^{\prime} b^{\prime}, a^{\prime \prime} b^{\prime \prime}}\left(\omega_{i}+\omega^{\prime}\right)
\end{aligned}
$$

To solve the individual integrals we assume that the function $\zeta^{a^{\prime} b^{\prime}, a^{\prime \prime} b^{\prime \prime}}\left(\omega_{i}+\omega^{\prime}\right)$ can be approximated as a Taylor expansion around $\omega_{i}$, truncated at first-order:

$$
\begin{gathered}
\zeta^{a^{\prime} b^{\prime}, a^{\prime \prime} b^{\prime \prime}}\left(\omega_{i}+\omega^{\prime}\right)=\sum_{p=0}^{\infty} \frac{\omega^{\prime}}{p !}\left(\frac{\mathrm{d}^{p} \zeta^{a^{\prime} b^{\prime}, a^{\prime \prime} b^{\prime \prime}}}{\mathrm{d} \omega^{\prime} p}\right)_{\omega_{i}} \\
\approx \zeta^{a^{\prime} b^{\prime}, a^{\prime \prime} b^{\prime \prime}}\left(\omega_{i}\right)+\omega^{\prime}\left(\frac{\mathrm{d} \zeta^{a^{\prime} b^{\prime}, a^{\prime \prime} b^{\prime \prime}}}{\mathrm{d} \omega^{\prime}}\right)_{\omega_{i}} .
\end{gathered}
$$

This leads to the approximate expression of the integrals

$$
\begin{aligned}
& \sum_{i=-N}^{N} \int_{-\Delta \omega / 2}^{\Delta \omega / 2} \mathrm{~d} \omega^{\prime} \gamma_{ \pm}^{a b}\left(\omega_{i}+\omega^{\prime}, v_{j}\right) \zeta^{a^{\prime} b^{\prime}, a^{\prime \prime} b^{\prime \prime}}\left(\omega_{i}+\omega^{\prime}\right) \\
& \approx \sum_{i=-N}^{N} \zeta^{a^{\prime} b^{\prime}, a^{\prime \prime} b^{\prime \prime}}\left(\omega_{i}\right) \int_{-\Delta \omega / 2}^{\Delta \omega / 2} \mathrm{~d} \omega^{\prime} \gamma_{ \pm}^{a b} \\
& \quad+\sum_{i=-N}^{N}\left(\frac{\mathrm{d} \xi^{a^{\prime} b^{\prime}, a^{\prime \prime} b^{\prime \prime}}}{\mathrm{d} \omega^{\prime}}\right) \int_{\omega_{i}}^{\Delta \omega / 2} \mathrm{~d} \omega^{\prime} \omega^{\prime} \gamma_{ \pm}^{a b}
\end{aligned}
$$

The remaining integrals can be solved analytically. From the definition of the $\gamma_{ \pm}^{a b}(\omega, v)$ function of Eq. (12), we have the following analytical solutions

$$
\begin{aligned}
& \int_{-\Delta \omega / 2}^{\Delta \omega / 2} \mathrm{~d} \omega^{\prime} \gamma_{ \pm}^{a b}\left(\omega^{\prime}+\omega_{i}, v_{j}\right) \\
& \quad=-\left(\operatorname{atan} q_{\mathrm{up}}-\operatorname{atan} q_{\mathrm{down}}\right) \pm \frac{i}{2} \log \left(\frac{\Gamma^{2}+q_{\mathrm{up}}^{2}}{\Gamma^{2}+q_{\mathrm{down}}^{2}}\right) \\
& \quad=g_{\mathrm{r}}(i, j) \pm i g_{i}(i, j)=g^{ \pm}(i, j) \\
& \int_{-\Delta \omega / 2}^{\Delta \omega / 2} \mathrm{~d} \omega^{\prime} \omega^{\prime} \gamma_{ \pm}^{a b}\left(\omega^{\prime}+\omega_{i}, v_{j}\right) \\
& \quad=\frac{\Gamma g_{i}(i, j)}{1-j \Delta v}+\left(\frac{\omega_{a b}}{1-j \Delta v}-\left(\omega_{0}+i \Delta \omega\right)\right) g_{\mathrm{r}}(i, j) \\
& \quad \pm i\left(\left[\frac{\omega_{a b}}{1-j \Delta v}-\left(\omega_{0}+i \Delta \omega\right)\right] g_{i}(i, j)+\frac{\Gamma}{1-j \Delta v} g_{\mathrm{r}}(i, j)+\Delta \omega\right) \\
& =g_{\mathrm{r}, \omega}(i, j) \pm i g_{i, \omega}(i, j)=g_{\omega}^{ \pm}(i, j)
\end{aligned}
$$

where

$q_{\text {up }}=\omega_{a b}-\left[\frac{\Delta \omega}{2}+\omega_{0}+i \Delta \omega\right](1-j \Delta v)$,

$q_{\text {down }}=\omega_{a b}-\left[-\frac{\Delta \omega}{2}+\omega_{0}+i \Delta \omega\right](1-j \Delta v)$.

We should note that our approach differs slightly from that of N\&W94; we use the analytical solutions to the integrals of Eq. (26) instead of assuming a sharply peaked function. Let us now insert these simplified integrals into the final approximate equation for the integral

$$
\begin{aligned}
\int \mathrm{d} \omega \gamma_{ \pm}^{a b}\left(\omega, v_{j}\right) \zeta^{a^{\prime} b^{\prime}, a^{\prime \prime} b^{\prime \prime}}(\omega) \approx & \sum_{i=-N}^{N}\left(\zeta^{a^{\prime} b^{\prime}, a^{\prime \prime} b^{\prime \prime}}\left(\omega_{i}\right) g^{ \pm}(i, j)\right. \\
& \left.+\left(\frac{\mathrm{d} \zeta^{\prime} b^{\prime}, a^{\prime \prime} b^{\prime \prime}}{\mathrm{d} \omega^{\prime}}\right)_{\omega_{i}} g_{\omega}^{ \pm}(i, j)\right)
\end{aligned}
$$

where the derivatives $\left(\frac{\mathrm{d} \zeta^{\prime} b^{\prime}, a^{\prime \prime} b^{\prime \prime}}{\mathrm{d} \omega^{\prime}}\right)_{\omega_{i}}$ can be evaluated via the finite-difference method. The numerical approximation for the integral over $v$ at a particular channel frequency $\omega_{i}$

$$
\left\langle\gamma_{ \pm}^{a b}\left(\omega_{i}\right) \rho_{k k^{\prime}}\right\rangle_{v}=\int \mathrm{d} v \gamma_{ \pm}^{a b}\left(\omega_{i}, v\right) \rho_{k k^{\prime}}(v)
$$

is obtained in a similar way, and yields

$$
\begin{aligned}
\int \mathrm{d} v \gamma_{ \pm}^{a b}\left(\omega_{i}, v\right) \rho_{k k^{\prime}}(v) \approx & \sum_{j=-N}^{N}\left(\frac{c_{0}}{\omega_{0}} \rho_{k k^{\prime}}\left(v_{j}\right) g^{ \pm}(i, j)\right. \\
& \left.-\left(\frac{c_{0}}{\omega_{0}}\right)^{2}\left(\frac{d \rho_{k k^{\prime}}}{d v^{\prime}}\right)_{v_{j}} g_{\omega}^{ \pm}(i, j)\right)
\end{aligned}
$$

where the derivatives $\left(\frac{\mathrm{d} \rho_{k k^{\prime}}}{\mathrm{d} v^{\prime}}\right)_{v_{j}}$, again, can be evaluated via the finite-difference method. We evaluated the accuracy of the truncated Taylor expansion, and found that adding higherorder terms had minimal effect. Using the numerical expressions of Eqs. (27) and (28) for the integrals, the density equations and propagation matrix can be set up. The latter will contain all seven propagation coefficients. Solving the density equations is the subject of the next subsection.

It should be noted that above we assumed that different frequency components of the radiation field are uncorrelated, which is a standard assumption in maser theory (Gray 2012). The same is true for the different velocity components of the molecular states. Numerical simulation of maser polarization propagation can be made using these formalisms by computing the state populations for a given radiation field (see next paragraph) with the use of Eq. (7) and by computing the propagation coefficients using Eq. (14) and the newly found state populations. Subsequently, the radiation field is propagated using Eq. (13), where for a small enough $\Delta s$ the propagated vector of Stokes parameters can be approximated by $\boldsymbol{I}(s+\Delta s, \omega)=e^{\Delta s \boldsymbol{K}(s, \omega)} \boldsymbol{I}(s, \omega)$, where $\boldsymbol{K}(s, \omega)$ stands for the matrix of propagation coefficients (see Eq. (13)). The initial radiation field may be blackbody radiation, and the initial guess for the state-populations $\sim \Lambda / \Gamma$. In the following paragraph, we put extra emphasis on the computation of the state populations.

\subsection{Solving the density equations}

Because convergence issues have been known to arise for the density equations of N\&W90 and N\&W94 at high maser saturation, we explicitly comment on the method we used to solve the density equations. In the following, we consider the density equations for $\mathrm{N} \& \mathrm{~W} 90$, but a similar methodology was used for the other approaches. From Eq. (21), we have $n_{F_{1}}^{2}+n_{F_{2}}^{2}$ coupled equations for the density matrix (for N\&W92, the dimensionality is reduced to $n_{F_{1}}+n_{F_{2}}$ ). To ensure the hermicity of the solutions, it is convenient to separate the density matrix elements into their real and imaginary parts,

$\rho_{a a^{\prime}}=\operatorname{Re}\left(\rho_{a a^{\prime}}\right)+i \operatorname{Im}\left(\rho_{a a^{\prime}}\right)$

and we require $\operatorname{Re}\left(\rho_{a a^{\prime}}\right)=\operatorname{Re}\left(\rho_{a^{\prime} a}\right)$ as well as $\operatorname{Im}\left(\rho_{a a^{\prime}}\right)=$ $-\operatorname{Im}\left(\rho_{a^{\prime} a}\right)$. We bundle the unique elements in the vector $\boldsymbol{\rho}=\left[\boldsymbol{\rho}_{a}, \boldsymbol{\rho}_{b}\right]^{T}$, where

$\boldsymbol{\rho}_{a}=\left[\rho_{11}^{(a)}, \rho_{22}^{(a)}, \cdots \rho_{n_{F_{1}} n_{F_{1}}}^{(a)}, \operatorname{Re}\left(\rho_{12}^{(a)}\right), \operatorname{Im}\left(\rho_{12}^{(a)}\right), \cdots, \operatorname{Im}\left(\rho_{n_{F_{1}}-1, n_{F_{1}}}\right)\right]$ 
and $\boldsymbol{\rho}_{b}$ is the analogous population vector for the lower state. We take the real and imaginary parts from Eq. (21) and find

$$
\begin{aligned}
\operatorname{Re}\left(\lambda_{a a^{\prime}}\right)= & -\Gamma \operatorname{Re}\left(\rho_{a a^{\prime}}\right)+\operatorname{Im}\left(\rho_{a a^{\prime}}\right) \omega_{a a^{\prime}}+\frac{2 \pi^{2}}{c \hbar^{2}} \\
& \times\left[\sum_{b b^{\prime}}\left(\operatorname{Re}\left(\rho_{b b^{\prime}}\right) \operatorname{Re}\left(\zeta^{a^{\prime} b, a b^{\prime}}\right)-\operatorname{Im}\left(\rho_{b b^{\prime}}\right) \operatorname{Im}\left(\zeta^{a^{\prime} b, a b^{\prime}}\right)\right)\right. \\
& -\sum_{b a^{\prime \prime}}\left(\operatorname{Re}\left(\rho_{a^{\prime \prime} a^{\prime}}\right) \operatorname{Re}\left(\zeta^{a b, a^{\prime \prime} b}\right)^{*}-\operatorname{Im}\left(\rho_{a^{\prime \prime} a^{\prime}}\right) \operatorname{Im}\left(\zeta^{a b, a^{\prime \prime} b}\right)^{*}\right) \\
& \left.-\sum_{b a^{\prime \prime}}\left(\operatorname{Re}\left(\rho_{a a^{\prime \prime}}\right) \operatorname{Re}\left(\left(\zeta^{a^{\prime} b, a^{\prime \prime} b}\right)^{*}\right)-\operatorname{Im}\left(\rho_{a a^{\prime \prime}}\right) \operatorname{Im}\left(\left(\zeta^{a^{\prime} b, a^{\prime \prime} b}\right)^{*}\right)\right)\right], \\
= & \boldsymbol{a}^{a a^{\prime}} \rho, \\
\operatorname{Im}\left(\lambda_{a a^{\prime}}\right)= & -\Gamma \operatorname{Im}\left(\rho_{a a^{\prime}}\right)-\operatorname{Re}\left(\rho_{a a^{\prime}}\right) \omega_{a a^{\prime}}+\frac{2 \pi^{2}}{c \hbar^{2}} \\
& \times\left[\sum_{b b^{\prime}}\left(\operatorname{Re}\left(\rho_{b b^{\prime}}\right) \operatorname{Im}\left(\zeta^{a^{\prime} b, a b^{\prime}}\right)+\operatorname{Im}\left(\rho_{b b^{\prime}}\right) \operatorname{Re}\left(\zeta^{a^{\prime} b, a b^{\prime}}\right)\right)\right. \\
& -\sum_{b a^{\prime \prime}}\left(\operatorname{Re}\left(\rho_{a^{\prime \prime} a^{\prime}}\right) \operatorname{Im}\left(\zeta^{a b, a^{\prime \prime} b}\right)^{*}+\operatorname{Im}\left(\rho_{a^{\prime \prime} a^{\prime}}\right) \operatorname{Re}\left(\zeta^{a b, a^{\prime \prime} b}\right)^{*}\right) \\
& \left.-\sum_{b a^{\prime \prime}}\left(\operatorname{Re}\left(\rho_{a a^{\prime \prime}}\right) \operatorname{Im}\left(\left(\zeta^{a^{\prime} b, a^{\prime \prime} b}\right)^{*}\right)+\operatorname{Im}\left(\rho_{a a^{\prime \prime}}\right) \operatorname{Re}\left(\left(\zeta^{a^{\prime} b, a^{\prime \prime} b}\right)^{*}\right)\right)\right], \\
= & \boldsymbol{b}^{a a^{\prime}} \boldsymbol{\rho} .
\end{aligned}
$$

The collection of density equations can thus be formulated in the following matrix equation

$$
\lambda=\boldsymbol{M} \rho \text {, }
$$

where all matrices and vectors are real, and we have the elements of the matrix

$\boldsymbol{M}=\left[\boldsymbol{a}_{11}, \boldsymbol{a}_{22}, \cdots \boldsymbol{a}_{n_{F_{1}} n_{F_{1}}}, \boldsymbol{a}_{12}, \boldsymbol{b}_{12}, \cdots, \boldsymbol{b}_{n_{F_{1}}-1, n_{F_{1}}}, \cdots\right]^{T}$,

where the last part is omitted, but is made up of the analogous density equations of the lower level. We can solve for all densities by

$\rho=\operatorname{inv}(\boldsymbol{M}) \lambda$.

The matrix inversion is performed using an LQ decomposition, taken from the standard LAPACK libraries (Anderson et al. 1999). This method is very robust, as exemplified by the fact that these density equations are solvable for arbitrary angular momentum transitions (matrix dimensionality) and maser saturation. This is in contrast to N\&W90 and N\&W94, where convergence problems were reported for transitions of $J>3$ (Nedoluha \& Watson 1990).

\subsection{Experiments}

We present the developed methods by using them to analyze masers with a non-paramagnetic Zeeman effect that have shown polarization in their emission. In the following, we only present results from the most rigorous N\&W94 method. We consider all Stokes parameters, high rates of stimulated emission, and nonZeeman polarizing mechanisms.

We report our calculations mainly through contour maps of the linear polarization degree $p_{\mathrm{L}}=\sqrt{Q_{0}^{2}+U_{0}^{2}} / I_{0}$, polarization angle $p_{a}=\operatorname{atan}\left(U_{0} / Q_{0}\right) / 2$, and circular polarization degree $p_{\mathrm{V}}=\left(V_{\max }-V_{\min }\right) / I_{0}$. The circular polarization degree is taken to be negative if $V_{\max }$ occurs at a frequency $\omega<\omega_{0}$. The Stokes parameters $I_{0}, Q_{0}$, and $U_{0}$ are taken at the peak of $I(\omega)$. The polarization angle is relative to the rejection of the magnetic field direction from the propagation direction, i.e., the magnetic field direction projected onto the plane of the sky.

\subsection{1. $\mathrm{SiO}$ masers}

We analyzed the polarization of $\mathrm{SiO}$ masers by a magnetic field. We ran simulations at various magnetic field strengths, angular momentum transitions, and propagation angles $\theta$. The molecular parameters that are used in the simulation are given in Table 1. We performed calculations for the $\mathrm{SiO}$ masers in the vibrational state $v=1$. SiO masers also occur in higher vibrational states. The results we present can be taken as similar to higher vibrational states. Only the different isotropic decay rates, which scale roughly as $\Gamma \approx 5 v \mathrm{~s}^{-1}$ (Elitzur 1992), lead to a different ratio $g \Omega / \Gamma$ which will have little impact on the presented results.

Maser polarization properties converge for $\omega_{D} \gg g \Omega$. To ensure $\omega_{D} \gg g \Omega$, we use a thermal maser width of $\Delta \omega_{\text {th }}=$ $1000 \times g \Omega \times J$, where $J$ is the angular momentum of the upper level. This thermal maser width corresponds to $v_{\text {th }} \approx$ $\frac{0.83 \times J^{2}}{B(\mathrm{G})} \mathrm{km} \mathrm{s}^{-1}$. We performed studies with the following:

- isotropic pumping, where the pumping matrix is $\boldsymbol{\Lambda}=\lambda \mathbf{1}$;

- polarized incident seed radiation, with isotropic pumping, but with seed radiation of $U / I=0.1$ and $U / I=0.5$;

- anisotropic pumping, where the pumping matrix characterized by Eq. (16). We ran simulations with moderate $\eta=0.1$ and high $\eta=0.5$ degrees of anisotropy. We ran simulations for three anisotropy directions: (i) parallel to the magnetic field, (ii) perpendicular to the magnetic field and propagation direction, and (iii) at $45^{\circ}$ from the magnetic field in the plane perpendicular to the propagation direction.

\subsubsection{Water masers}

We present the polarization of water masers in the parameter space relevant to observations. The strongest water masers do not exceed $T_{b} \Delta \Omega=10^{13} \mathrm{Ksr}$ (Garay et al. 1989; Sobolev et al. 2018) and magnetic field estimates range from $B=1 \mathrm{mG}-1 \mathrm{G}$. The thermal width of the maser molecules affects the maser polarization (N\&W92), so we analyzed the water masers excited at different temperatures. Preferred hyperfine pumping is a possibility for this maser species, so we analyzed a range of relevant cases. We also explored the effect of alternative polarization mechanisms on the polarization of water masers.

\section{Results}

We report here the results of representative numerical simulations of several $v=1 \mathrm{SiO}$ masers and the $22 \mathrm{GHz}$ water maser. Results are only reported for the most rigorous N\&W94 approach. We divide up this section into experiments on $\mathrm{SiO}$ and water masers, and further compartmentalize experiments of isotropically pumped masers, masers with polarized seed radiation, and anisotropically pumped masers. The results are graphically summarized as polarization landscapes, dependent on maser luminosity and propagation magnetic field angle. A large part of the results can be found in the Appendix. In this section we lay out observable patterns in the reported polarization landscapes; in the following section, we discuss the physical processes that give rise to these patterns.

\subsection{SiO masers}

\subsubsection{Isotropic pumping}

Simulations of a $J=1-0 \mathrm{SiO}$ maser in a $1 \mathrm{G}$ magnetic field with varying luminosity and magnetic field angle are given in Fig. 1. Simulations of higher angular momentum and at different 
Table 1. Molecular parameters for $v=1 \mathrm{SiO}$ masers.

\begin{tabular}{cccccc}
\hline \hline & $v_{0}(\mathrm{GHz})$ & $g \Omega_{\mathrm{up}} / B\left(\mathrm{~s}^{-1} \mathrm{mG}^{-1}\right)$ & $g \Omega_{\mathrm{down}} / B\left(\mathrm{~s}^{-1} \mathrm{mG}^{-1}\right)$ & $A_{i j}\left(\mathrm{~s}^{-1}\right)$ & $\Gamma\left(\mathrm{s}^{-1}\right)$ \\
\hline$J=1-0$ & 43.122 & 0.75 & 0.75 & $3.024 \times 10^{-6}$ & 5 \\
$J=2-1$ & 86.243 & 0.75 & 0.75 & $2.903 \times 10^{-5}$ & 5 \\
$J=3-2$ & 129.363 & 0.75 & 0.75 & $1.050 \times 10^{-4}$ & 5 \\
$J=4-3$ & 172.481 & 0.75 & 0.75 & $2.580 \times 10^{-4}$ & 5 \\
$J=5-4$ & 215.595 & 0.75 & 0.75 & $5.134 \times 10^{-4}$ & 5 \\
\hline
\end{tabular}

Table 2. Molecular parameters for the $22.235 \mathrm{GHz}$ water maser.

\begin{tabular}{llllll}
\hline & $\Delta \nu_{\text {hyp }}(\mathrm{kHz})$ & $g \Omega_{\mathrm{up}} / B\left(\mathrm{~s}^{-1} \mathrm{mG}^{-1}\right)$ & $g \Omega_{\mathrm{down}} / B\left(\mathrm{~s}^{-1} \mathrm{mG}^{-1}\right)$ & $A_{i j}\left(\mathrm{~s}^{-1}\right)$ & $\Gamma\left(\mathrm{s}^{-1}\right)$ \\
\hline$F=5-4$ & -33.38 & -0.79 & -1.34 & $1.789 \times 10^{-9}$ & 1 \\
$F=6-5$ & 0 & 3.71 & 4.12 & $1.806 \times 10^{-9}$ & 1 \\
$F=7-6$ & 43.018 & 6.51 & 7.24 & $1.860 \times 10^{-9}$ & 1 \\
\hline
\end{tabular}

magnetic fields are given in Figs. A.1-A.3. The only polarizing entity in these simulations is the magnetic field and its interaction with the directional maser radiation. We observe, regardless of the magnetic field strength or angular momentum of the transition, a peak in the linear polarization fraction around $\log (R / g \Omega)=0$, which is in the region where the rate of magnetic precession $(g \Omega)$ and stimulated emission rate $(R)$ become comparable in size. The peak of the linear polarization fraction is on the order of the GKK73 estimate of linear polarization fraction, but can exceed it by $10 \%$. This excess of polarization is associated with significant polarization in the Stokes $U$ spectrum, and is most pronounced for strong magnetic fields and around $\theta=20^{\circ}$. The linear polarization fraction increases with the magnetic field strength, and decreases with the angular momentum $J$, of the transition. A large region, around $\log (R / g \Omega)=0-0.5$, $0-1.5$, and $0-2.5$, for $B=100 \mathrm{mG}, 1 \mathrm{G}$, and $10 \mathrm{G}$ has a stable polarization fraction of about $p_{\mathrm{L}}=1 / 3$ (for the $J=1-0$ transition) for a wide range of angles. The stability of the polarization fraction over $R / g \Omega$ correlates with the propagation angle and magnetic field strength. For $\theta$ close to $90^{\circ}$, and strong magnetic fields, the polarization fraction is stable for a wide range of $R / g \Omega$. Significant polarization occurs for a much greater region of $R$ and $\theta$ when the magnetic field strength is increased. We note that the polarization fraction function fulfills the symmetry relation: $p_{\mathrm{L}}(\theta)=p_{\mathrm{L}}\left(180^{\circ}-\theta\right)$. The polarization angle and circular polarization flip according to $p_{\mathrm{a}}(\theta)=-p_{\mathrm{a}}\left(180^{\circ}-\theta\right)$ and $p_{\mathrm{V}}(\theta)=-p_{\mathrm{V}}\left(180^{\circ}-\theta\right)$. An interesting feature is found near the magic angle, where for $\log (R / g \Omega) \lesssim 0$ a sharp drop in the polarization fraction is observed that becomes more pronounced with decreasing $\log (R / g \Omega)$. Polarization around the magic angle for $\log (R / g \Omega) \lesssim-2$ is mostly absent.

Directing our attention to the polarization angles, we observe that the $90^{\circ}$ flip of the polarization angle can be produced by crossing the magic angle $\theta_{\mathrm{m}}$, and by the transition from $\log (R / g \Omega) \ll 0$ to $\log (R / g \Omega) \gg 0$. The $\theta_{\mathrm{m}}$ crossing polarization angle flip becomes sharper with $B$, and manifests itself only for $\log (R / g \Omega)<-1$. For higher $\log (R / g \Omega)$ the flip will get less sharp. These features are particularly clear in Fig. 2 . In the intermediate region around $\log (R / g \Omega)$, the region of highest linear polarization, arbitrary polarization angles can be produced. Overall, apart from the sharper $90^{\circ}$ flip at $\theta_{\mathrm{m}}$, the polarization angle as a function of $\log (R / g \Omega)$ and $\theta$ is very consistent for the different magnetic field strengths and different transitions. At $\log (R / g \Omega) \gg 1$, the polarization vectors will be aligned $(\theta=0)$ with the (projected) magnetic field direction at any propagation angle $\theta$.

We continue by analyzing the landscape of circular polarization. We observe that the highest circular polarization fractions occur around $\theta=20^{\circ}$, and is associated with the region of maximum linear polarization fractions. However, for circular polarization maximum polarization occurs at slightly higher $R$. Circular polarization is most significant between $\log (R / g \Omega)>-1$ and $\log (R / g \Omega)<2.5$, and quickly drops to zero for $\theta \rightarrow 90^{\circ}$. Circular polarization contours for other magnetic field strengths (see Appendix A) show similar circular polarization landscapes. The maximum circular polarization fraction does not change much for stronger magnetic field strengths, although the region of significant polarization becomes larger. We saw an analogous effect for the linear polarization. Instead, lower magnetic field strength decreases the maximum circular polarization fraction, and also decreases the region of significant circular polarization. For these simulations, we chose a thermal width $\Delta \omega$, such that $\Delta \omega=1000 \mathrm{~g} \Omega\left(v_{\mathrm{th}}^{\mathrm{SiO} J=1-0}=0.033 \frac{B}{\mathrm{mG}} \mathrm{km} \mathrm{s}^{-1}\right)$, and found that variations in the thermal width did not yield significantly different circular polarization values as long as the requirement $\Delta \omega \gg g \Omega$ was fulfilled ${ }^{2}$.

Simulations of the $J=2-1 \mathrm{SiO}$ maser-transition reveal a sharp drop in both linear and circular polarization fractions with respect to the $J=1-0$ transitions. The maxima of the polarization fractions are $p_{Q_{\max }}=0.20$ and $p_{\mathrm{V}_{\max }}=0.10$ for $B=1 \mathrm{G}$, constituting a $60 \%$ loss in polarization with respect to the $J=1-0$ transition. The general shapes of the contour maps are retained. The $90^{\circ}$ flip, caused by an increase in $R$, characteristic of the $\theta<\theta_{\mathrm{m}}$ masers, is observed to be less sharp, and occurs at higher $\log (R / g \Omega)$. Going to higher angular momentum transitions, the changes become less pronounced with respect to the $J=2-1$ transition, although we do observe a minor but steady loss in polarizing strength of the maser with increasing $J$.

We also investigate the spectral properties of the $\mathrm{SiO}$ maser polarization. In Fig. 3, we report three spectra of $J=1-0, B=$ $100 \mathrm{mG}$, isotropically pumped $\mathrm{SiO}$ masers at $\log (R / g \Omega)=-1,0$, and 1. In the figure, all the Stokes parameters are plotted, as are the polarization angles across the spectrum. We note that the

\footnotetext{
2 These remarks are concerned with the polarizing mechanism around $-2<\log (R / g \Omega)<2$. As is discussed later, circular polarization can be introduced via pure spectral decoupling of the $\Delta m= \pm 1$ transitions. Circular polarization via such a mechanism is dependent on the line width and thus maser thermal width.
} 


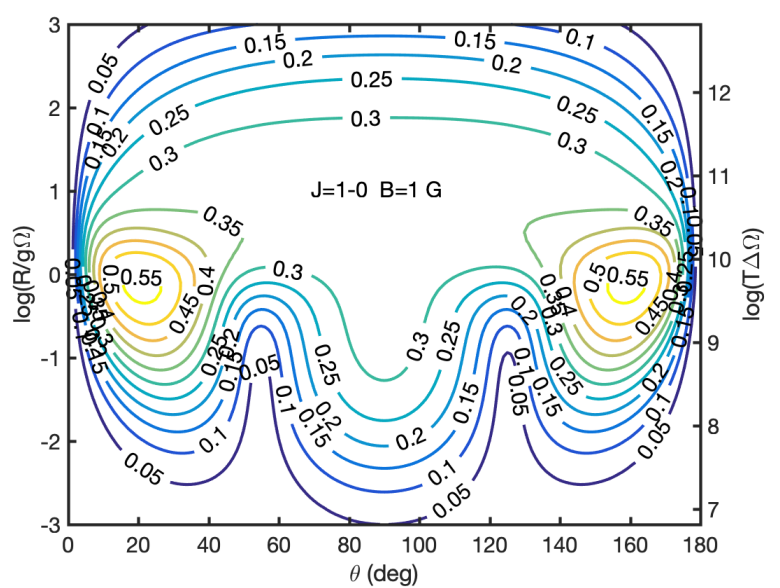

(a)

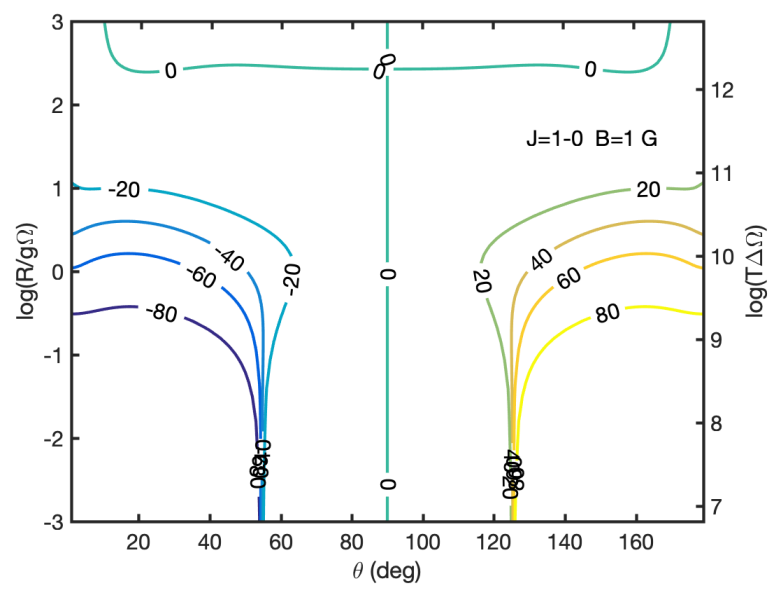

(b)

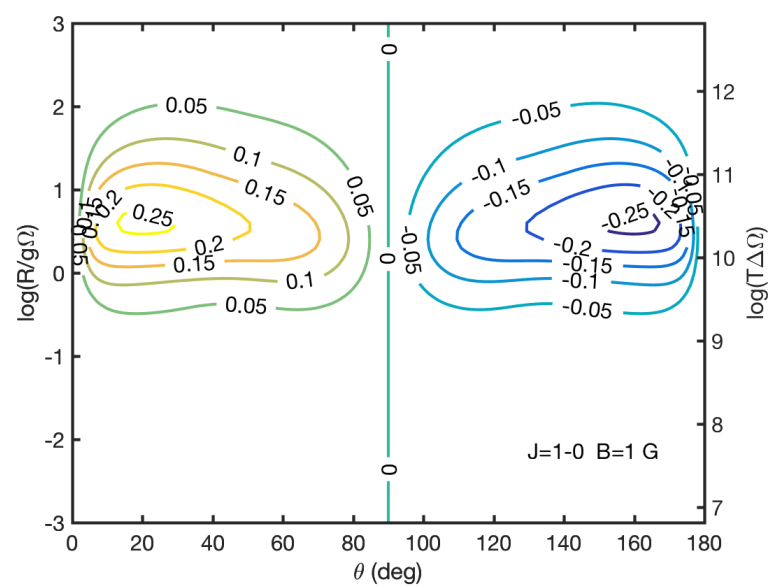

(c)

Fig. 1. Contour polarization plots of $v=1 \mathrm{SiO}$ masers. The linear polarization fraction (a), angle (b), and circular polarization fraction (c) are plotted as a function of the propagation angle $\theta$ and the rate of stimulated emission. Magnetic field strength and transition angular momentum are indicated. For simulations with $J_{\text {up }}>1$ and other magnetic field strengths, see Figs. A.1-A.3

spectrum broadens with $R$ because we already passed the saturation level at $\log (R / g \Omega)=-1$. With the broadening, though, the Stokes $V$ fraction does not decrease as would be expected from a local thermodynamic equilibrium (LTE) analysis. The linear polarization roughly follows the same spectral form as

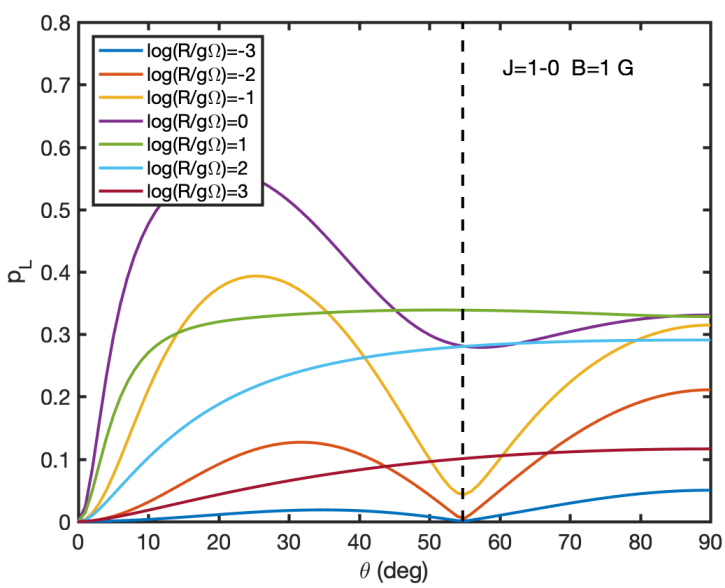

(a)

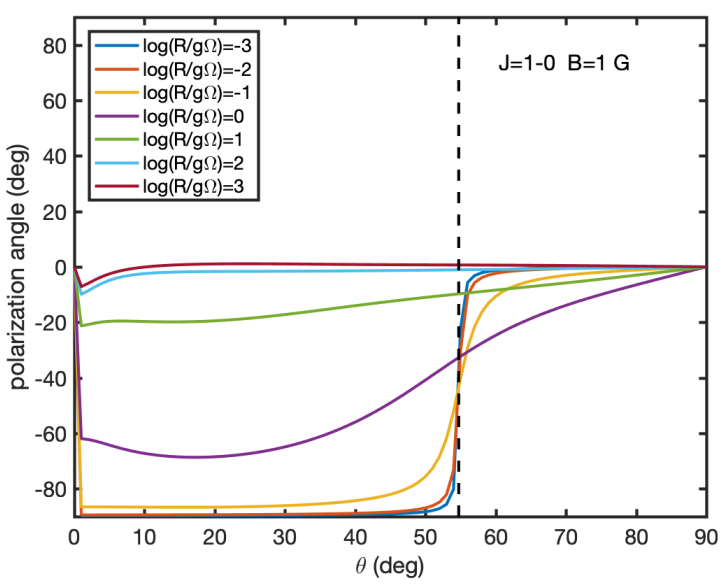

(b)

Fig. 2. Linear polarization fraction (a) and angle (b) of isotropically pumped $v=1 \mathrm{SiO}$ masers as a function of the propagation angle $\theta$ for different saturation rates. The sharp $90^{\circ}$ flip of the polarization at the magic angle (black dotted line) becomes less sharp and disappears with increasing levels of saturation. Magnetic field strength and transition angular momentum are indicated.

the Stokes $I$ spectrum, and the polarization angle can change by up to $\sim 30^{\circ}$ across the spectrum. We also note the perfect anti-symmetrical nature of the Stokes $V$ spectrum, as is expected from a LTE analysis, which is retained for all $R$.

\subsubsection{Polarized incident radiation}

Simulations of the polarization of a $J=1-0 \mathrm{SiO}$ maser at $B=1 \mathrm{G}$ with partially polarized seed radiation are shown in Figs. 4 and 5. Simulations of higher angular momentum and at different magnetic fields are given in Figs. A.4-A.9. When analyzing these types of masers, a distinction should be made between the regime of weak maser emission, where the rate of stimulated emission is significantly weaker than the magnetic field $(\log (R / g \Omega)<-2)$, and the regime of strong maser emission, where the two quantities are comparable in size. In the weak maser regime, the incident polarized radiation is simply amplified and the fractional polarization from the incident radiation is retained, along with the polarization angle of the incident radiation. In the strong maser regime, we found distinct differences in the polarization landscapes between the strongly 


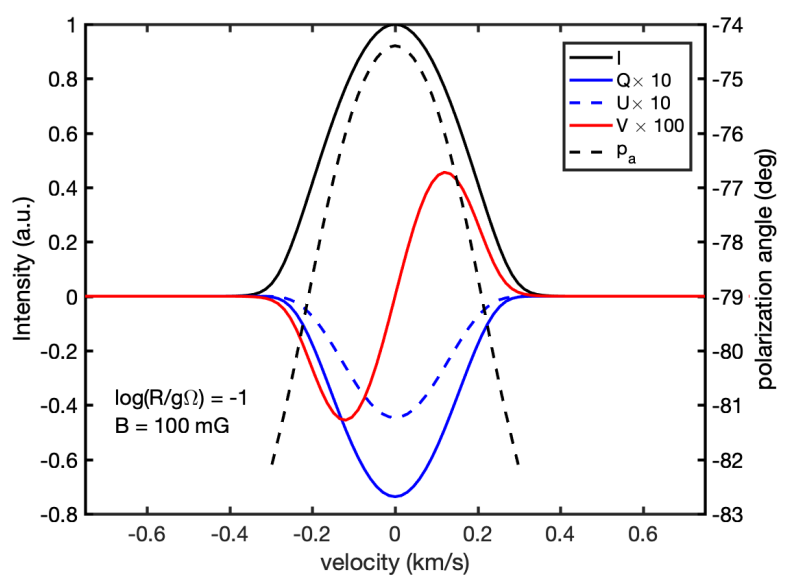

(a)

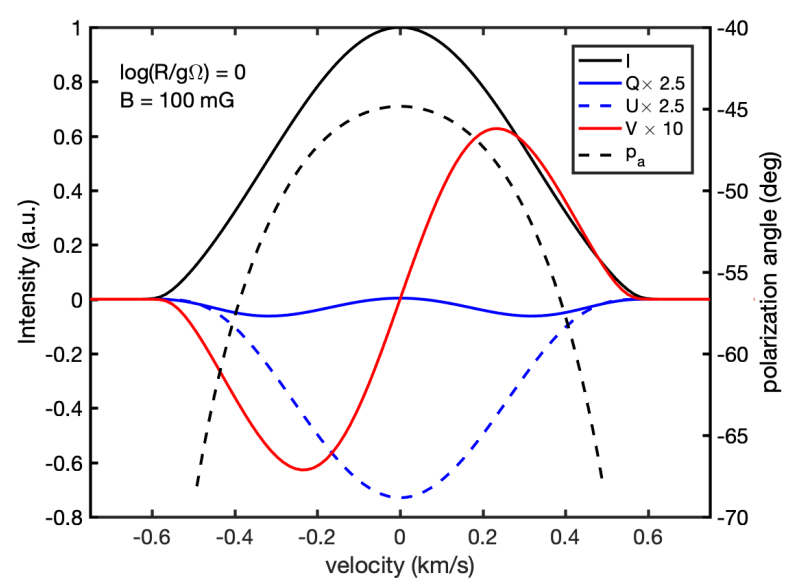

(b)

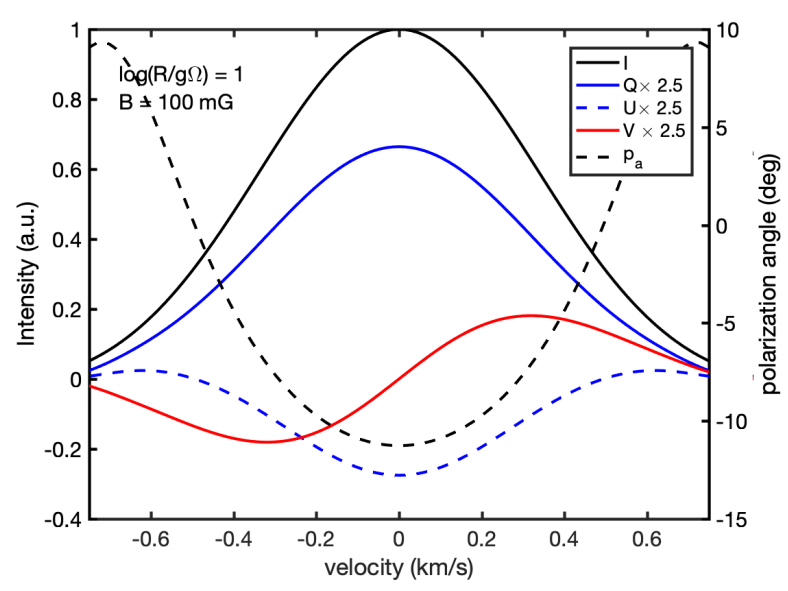

(c)

Fig. 3. $\mathrm{SiO} J=1-0$ maser spectra for different levels of saturation. All Stokes parameters (left $y$-axis) and the polarization angles (right $y$-axis) are plotted. The polarization angle is defined with respect to the magnetic field direction projected on the plane of the sky. Simulations were carried out at $B=100 \mathrm{mG}$, with a magnetic field propagation angle of $\theta=45^{\circ}$.

polarized $(U / I=0.5)$ and the weakly polarized $(U / I=0.1)$ incident radiation. The linear and circular polarization landscapes of the weakly polarized incident radiation above $\log (R / g \Omega)>0$ look very similar to the landscapes generated from isotropic seed radiation. In contrast, the linear polarization landscape of the strongly polarized incident seed radiation looks completely different, and only converges to the landscape of isotropic seed radiation for $\log (R / g \Omega)>2$. Interestingly, the effects on the circular polarization landscapes are rather small, even for the strongly polarized incident seed radiation. Although the effects are small, we observe an increase in circular polarization fraction with the polarized incident seed radiation.

Around the magic angle $\theta_{\mathrm{m}}$, the incident polarization fraction is retained for the highest $R$. The strongest linear polarization fraction is found around $\theta=20^{\circ}$ and where $R \sim g \Omega$, just as we have seen for isotropic seed radiation. Although we should note that the maximum linear polarization fraction occurs for somewhat lower $R$, which is an effect that is most pronounced at the strongly polarized seed radiation. We should also note that the symmetry around $\theta=90^{\circ}$ that characterizes the simulations with isotropic seed radiation is not retained by these simulations. The preferred direction of the incident radiation breaks the symmetry. This is perhaps most strongly reflected in the polarization angle maps. Here a feature is seen in the maps for both strongly and weakly polarized incident radiation, at the magic angle $\theta=\theta_{\mathrm{m}}$, and around $g \Omega \sim R$, where a range of different angles come together. Additionally, for $\theta<\theta_{\mathrm{m}}$, a large and sharp polarization angle change is seen around $\log (R / g \Omega) \sim-1$. Further inspection of these fluctuations in the polarization angle reveal that in this region, the initially positive Stokes $U$ element of the radiation drops and changes sign. For $\theta<\theta_{\mathrm{m}}$, the Stokes $Q$ coefficient initially builds up as negative, but turns positive after $\log (R / g \Omega) \sim 0$. For $\theta>\theta_{\mathrm{m}}$, the Stokes $Q$ coefficient does not become negative. For angles $\theta>90^{\circ}$, the Stokes $U$ element of the radiation retains its positive sign throughout the propagation.

At different magnetic field strengths, similar general features are observed that were also pointed out in the isotropic seed-radiation simulations. For instance, we observed that the magnetic field strength is correlated to the area $(\theta$ vs. $R)$ of significant polarization. An interesting feature is that the lower magnetic field strength simulations seem to be more affected by the incoming radiation than the stronger magnetic field strength simulations, which retain more of the general structure also observed for the isotropic seed radiation. Just as for the isotropic seed-radiation masers, the higher angular momentum transitions are significantly less polarized. However, for the higher angular momentum contours, the general structure of polarization contours is strongly influenced by the incoming polarized radiation. The simulations with strongly polarized incoming radiation, have nearly no general dependence on $\theta$, as the incoming (linear) polarization fraction smoothly deteriorates from $\log (R / g \Omega)>0$, to nullify around $\log (R / g \Omega) \sim 3$. These effects are also reflected in the landscape of circular polarization, which is affected by the highly polarized incoming radiation. Although the effects are not as pronounced as in the linear polarization contours, and do not cause high fractions of circular polarization.

\subsubsection{Anisotropic pumping}

When anisotropic pumping is in play, the distinction should be made between strong masers, where the radiation significantly influences the direction of the molecule $(R \gtrsim g \Omega)$, and weak masers, where this is not the case. Weak masers propagating through an anisotropically pumped medium, will accrue polarization monotonically. The polarization will rise until the point where the radiative interaction becomes stronger than the degree of anisotropic pumping. After this point, the polarization degree will drop, and the standard magnetic field polarization mechanism will take over as the main source of polarization. 


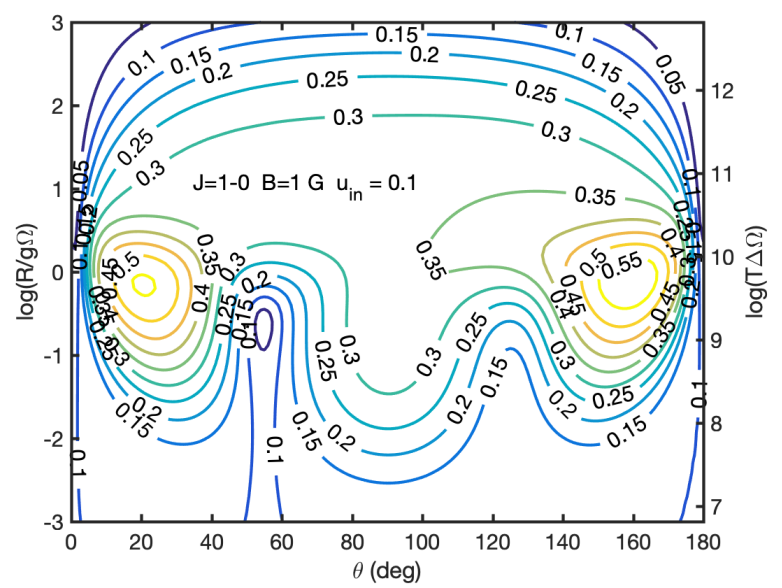

(a)

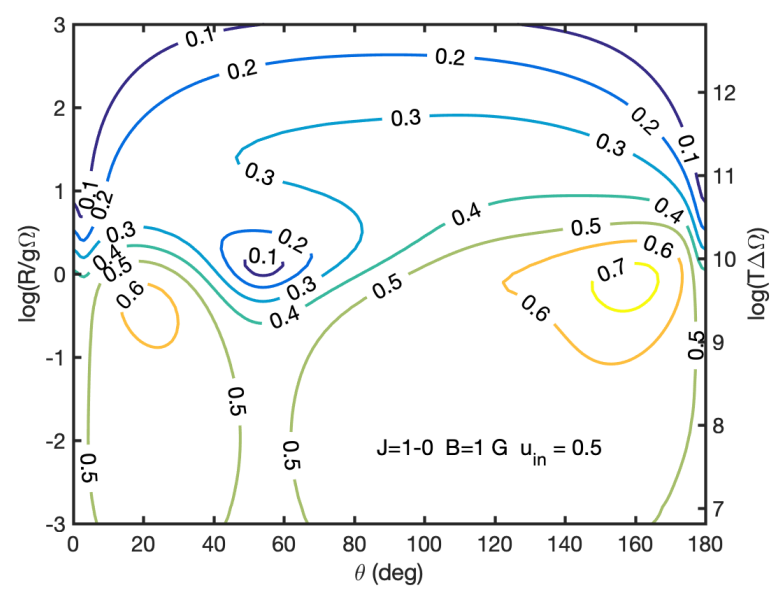

(c)

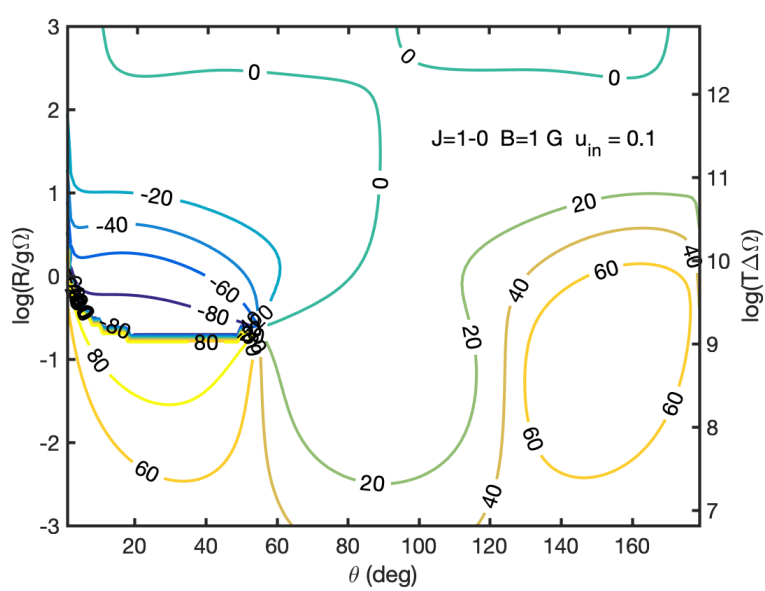

(b)

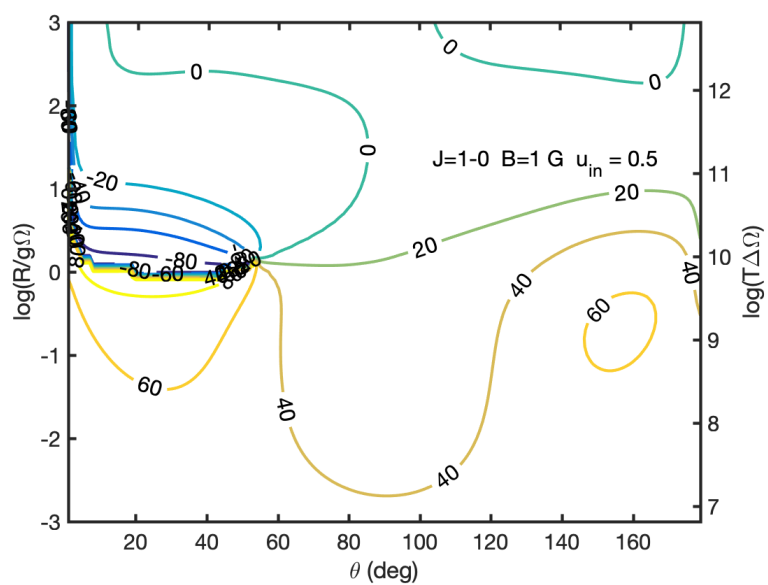

(d)

Fig. 4. Contour plots of the linear polarization fraction and angle of an $\mathrm{SiO}$ maser as a function of the propagation angle $\theta$ and the rate of stimulated emission. Maser simulations performed with incident polarized radiation of (a,b) $U / I=0.1$ and (c,d) $U / I=0.5$. Magnetic field strength and transition angular momentum are indicated. For simulations with $J_{\text {up }}>1$ and other magnetic field strengths, see Figs. A.4-A.9 in the Appendix.

Figure 6 shows the polarization of anisotropically pumped $\mathrm{SiO}$ masers with varying intensity of seed radiation as a function of the rate of stimulated emission.

The polarization of weak masers is independent of the magnetic field strength, but will be highly dependent on the intensity of the seed radiation, and on the anisotropy of the pumping, $\eta$. Strong masers have magnetic field interaction as their main polarization mechanism, but they are still influenced by anisotropic pumping, especially in the transitory period between the weak and strong maser. The polarization of the strongest masers is independent of the intensity of the incoming radiation.

The polarization landscape of an anisotropically pumped $\mathrm{SiO}$ maser at $B=1 \mathrm{G}$ is plotted in Fig. 7. Simulations of higher angular momentum and at different magnetic fields are given in Figs. A.10-A.18. If we examine the weak maser region, we see directly a strong decline in polarization for $\theta \rightarrow 0$. For higher rates of stimulated emission, at $\log (R / g \Omega)>1$, we see that the polarization is similar to the polarization generated by an isotropically pumped maser (Fig. 1), although we observe additional polarization in the regions around $\theta=90^{\circ}$ and $R \sim g \Omega$. Also, we actually observe a decrease in polarization in the region around $\theta=20^{\circ}$ and $R \sim g \Omega$ with respect to the isotropically pumped maser. However, if the anisotropy param- eter is increased the resemblance to the isotropically pumped maser vanishes rapidly, and arbitrarily high polarization can be achieved.

We observe that for increasing angular momentum of the transition, the same anisotropy parameter $\eta$ yields a weaker polarization buildup in the weak maser regime. Nevertheless, a high fractional linear polarization can be achieved for the higher angular momentum transitions as a result of the anisotropic pumping. A sufficiently large anisotropy parameter can yield polarizations as high as $100 \%$.

The orientation of the anisotropy in Fig. 7 is perpendicular to both the magnetic field direction $\boldsymbol{b}$ and the propagation direction $\boldsymbol{s}$. In this orientation, the polarization maps are symmetric, such that $p_{\mathrm{L}}(\theta)=p_{\mathrm{L}}\left(180^{\circ}-\theta\right), p_{\mathrm{a}}(\theta)=-p_{\mathrm{a}}\left(180^{\circ}-\theta\right)$, and $p_{\mathrm{V}}(\theta)=$ $-p_{\mathrm{V}}\left(180^{\circ}-\theta\right)$. This symmetry will be broken, however, when the direction of the anisotropy orients itself in the plane such that $\boldsymbol{b}$ spans with $\boldsymbol{s}$ (see Appendix A).

We now consider the circular polarization of the anisotropically pumped $\mathrm{SiO}$ maser. We found there was some influence of the anisotropic pumping on the circular polarization, but the structure is mostly similar to that obtained from isotropic pumping, and the enhancement of polarization is not as strong as it was for the linear polarization analogues. Comparing two 


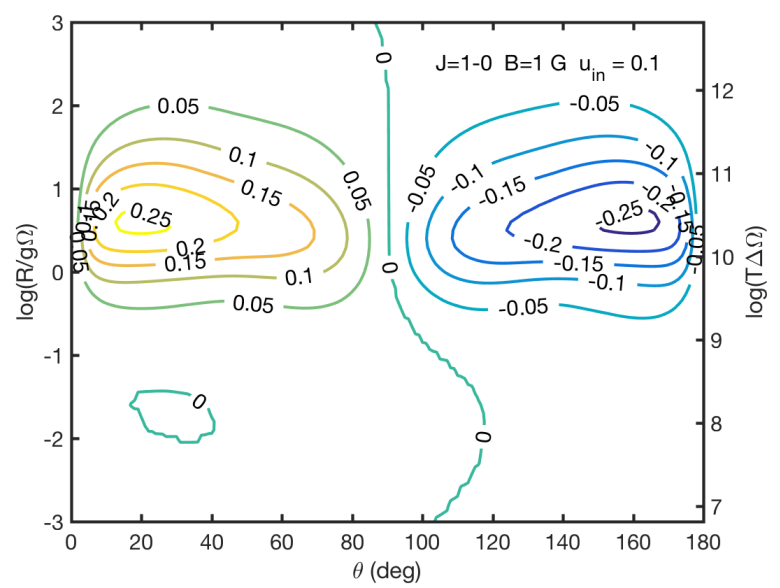

(a)

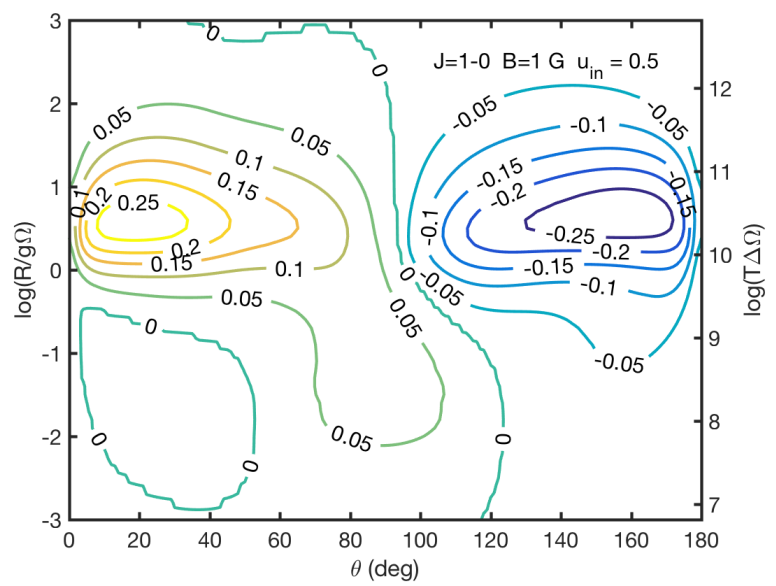

(b)

Fig. 5. Contour plots of the circular polarization fraction of the $\mathrm{SiO}$ maser as a function of the propagation angle $\theta$ and the rate of stimulated emission. Maser simulations performed with incident polarized radiation of (a) $U / I=0.1$ and (b) $U / I=0.5$. Magnetic field strength and transition angular momentum are indicated.

orientations of the anisotropy directions, $\boldsymbol{a}_{1} \perp \boldsymbol{b}, \boldsymbol{s}$ and $\boldsymbol{a}_{2} \| \boldsymbol{b}$, we find that the anisotropic pumping in the $\boldsymbol{a}_{1}$ direction actually lowers the circular polarization, while pumping in the $\boldsymbol{a}_{2}$ direction enhances it. In the weak maser regime there is no large circular polarization fraction, nor does the fraction depend on the brightness of the seed radiation.

\section{2. $\mathrm{H}_{2} \mathrm{O}$ masers}

\subsubsection{Isotropic pumping}

We examined the regime of magnetic fields from $B=20 \mathrm{mG}$ to $B=100 \mathrm{mG}$, at $v_{\text {th }}=0.6 \mathrm{~km} \mathrm{~s}^{-1}(T=260 \mathrm{~K})$ to $v_{\text {th }}=3.0 \mathrm{~km} \mathrm{~s}^{-1}$ $(T=6500 \mathrm{~K})$. We summarize the results of these simulations in Fig. 8, and further results can be found in Figs. A.19 and A.20. The linear polarization fraction for these water masers is only appreciable from about $T_{b} \Delta \Omega=10^{10} \mathrm{Ksr}$, or $\log (R / g \Omega)>$ -1.5 , where the strongest masers display the strongest polarization. The magnetic field interaction term is not strong enough to facilitate the large overshoot in polarization around $\theta=20^{\circ}$ that we saw earlier. Rather, the maximum linear polarization is found around $\theta \rightarrow 90^{\circ}$. In the range from $B=20 \mathrm{mG}$ to $B=100 \mathrm{mG}$, the linear polarization of the water masers does not

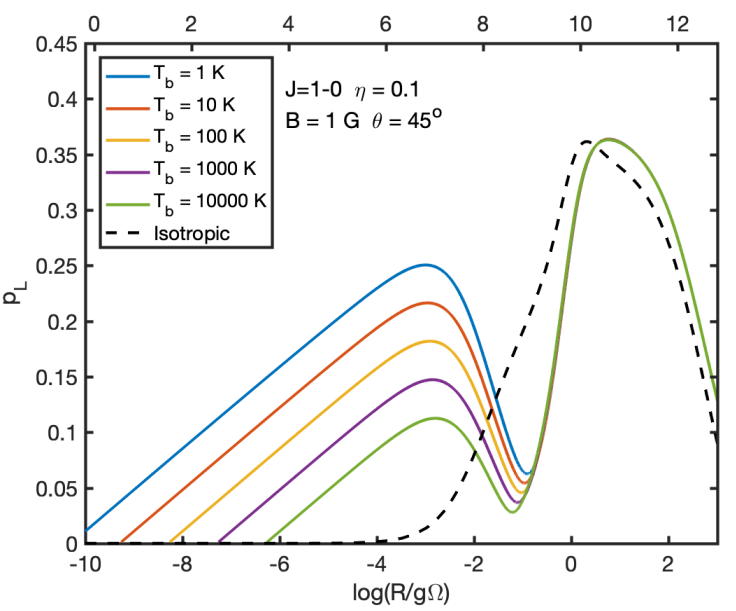

(a)

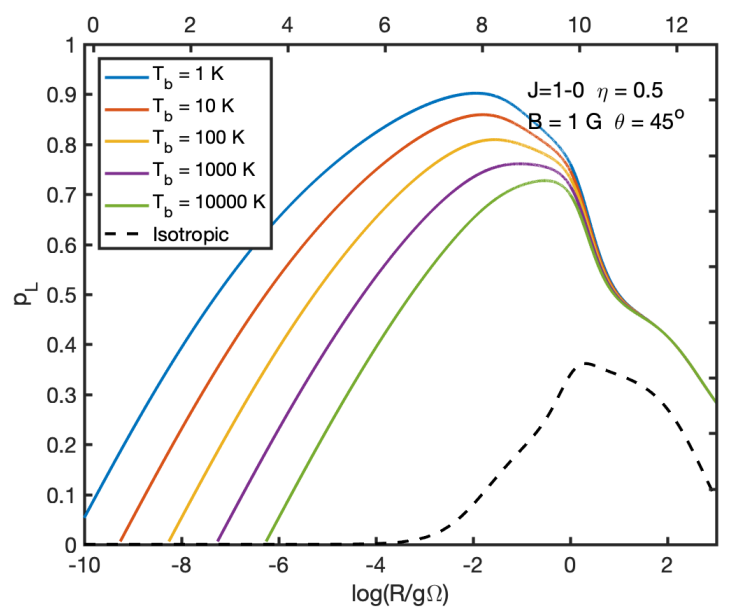

(b)

Fig. 6. Plots of the linear polarization fraction of anisotropically pumped $\mathrm{SiO}$ masers as a function of the rate of stimulated emission. Maser simulations performed with anisotropy parameters of (a) $\eta=0.1$ and (b) $\eta=0.5$. Simulations are performed for a range of incident radiation strengths, as indicated in the figure. Magnetic field strength, propagation angle, and transition angular momentum are indicated.

change significantly, although there is a slight general increase in linear polarization fraction. For simulations at higher thermal widths, $v_{\text {th }}>1 \mathrm{~km} \mathrm{~s}^{-1}$, there is no significant effect on the linear polarization fraction. For $v_{\text {th }}<1 \mathrm{~km} / \mathrm{s}$ we observe minor effects as the lines are no longer completely blended. For these simulations, polarization will start at higher maser intensity, but will soon converge to the landscape of the other $v_{\text {th }}$ solutions, as broadening of the maser blends the individual lines. Analysis of the polarization angle maps reveal no significant difference between different magnetic field strengths or between different thermal widths. The most striking feature of the polarization angle maps are the sharp $90^{\circ}$ flips associated with crossing the magic angle that are general for any $T_{b} \Delta \Omega$. We observe another sharp angle flip, around $\log (R / g \Omega) \sim 0.75$ for $\theta<\theta_{\mathrm{m}}$, but this is a $180^{\circ}$ flip.

The circular polarization maps present a rather complicated landscape of circular polarization, never quite reaching high degrees of circular polarization. Weaker masers with $T_{\mathrm{b}} \Delta \Omega \ll 10^{11} \mathrm{Ksr}$, follow roughly the LTE estimate of the circular polarization $p_{\mathrm{V}} \propto 2 A_{F F^{\prime}} B_{\text {Gauss }} \cos \theta / \Delta v_{F}(\mathrm{~km} / \mathrm{s}$ ) (Fiebig \& Güsten 1989). For these masers we observe the strongest circular 


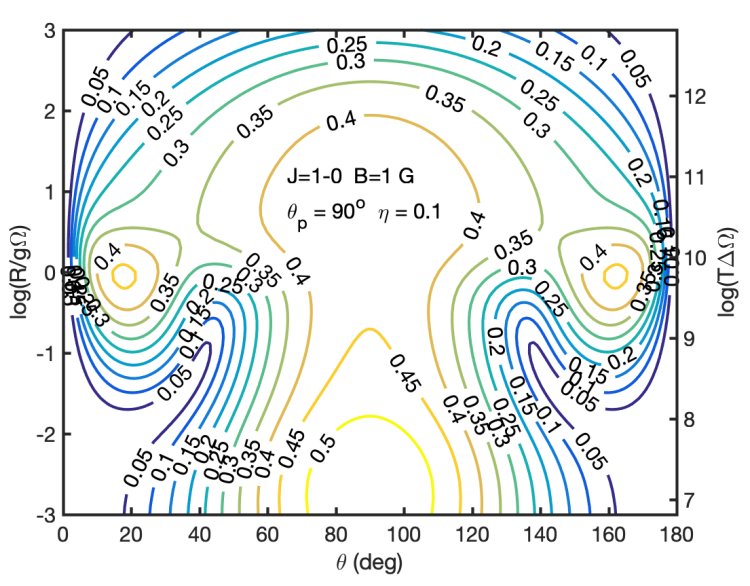

(a)

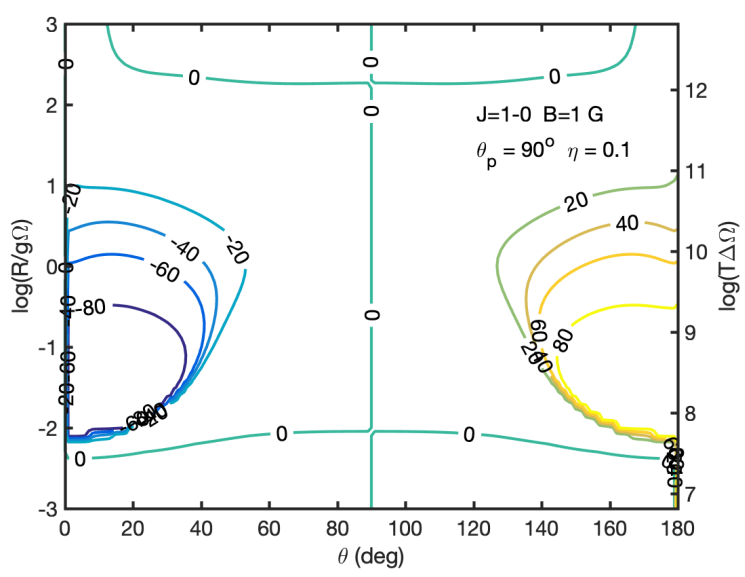

(b)

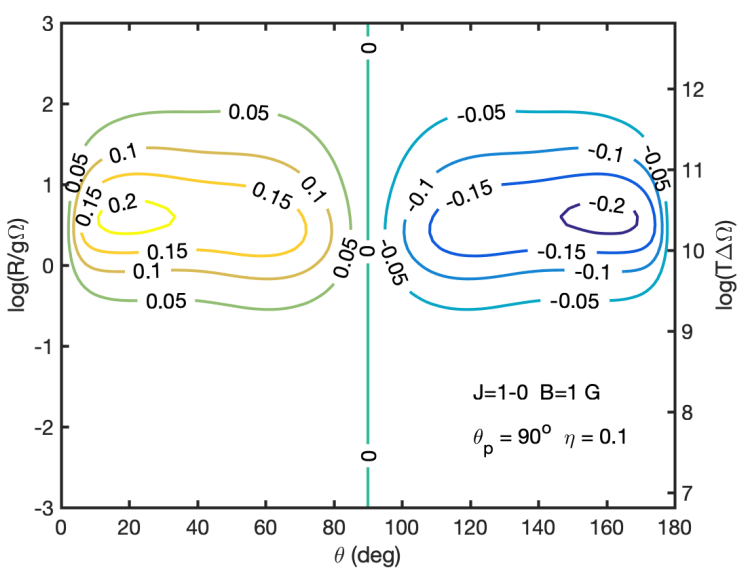

(c)

Fig. 7. Contour plot of the (a) linear polarization fraction, (b) polarization angle, and (c) circular polarization fraction of anisotropically pumped $\mathrm{SiO}$ masers as a function of the rate of stimulated emission and the angle $\theta$. Maser simulations were performed with anisotropy parameters of $\eta=0.1$ and at an anisotropy angle perpendicular to the magnetic field and propagation direction. Simulations were performed for an incident radiation-strength of $T_{b}=0.1 \mathrm{~K}$, but here only a small part of the region sensitive to this parameter is shown. Magnetic field strength and transition angular momentum are indicated. For simulations with $J_{\text {up }}>1$ and other magnetic field strengths, see Figs. A.10-A.18 in the Appendix.

polarization for $\theta \rightarrow 0^{\circ}$ and low $v_{\text {th }}$, which gradually diminishes for higher $v_{\text {th }}$ and angles $\theta \rightarrow 90^{\circ}$. When $T_{b} \Delta \Omega>10^{9} \mathrm{Ksr}$, the

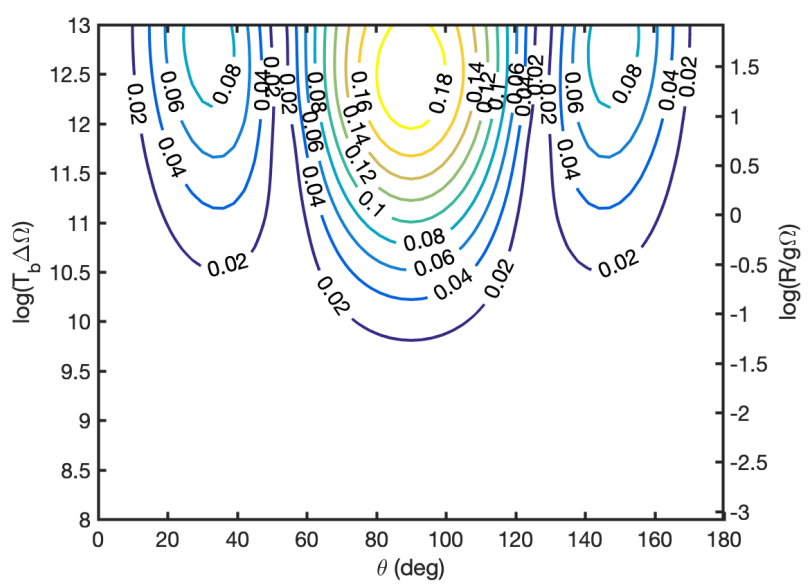

(a)

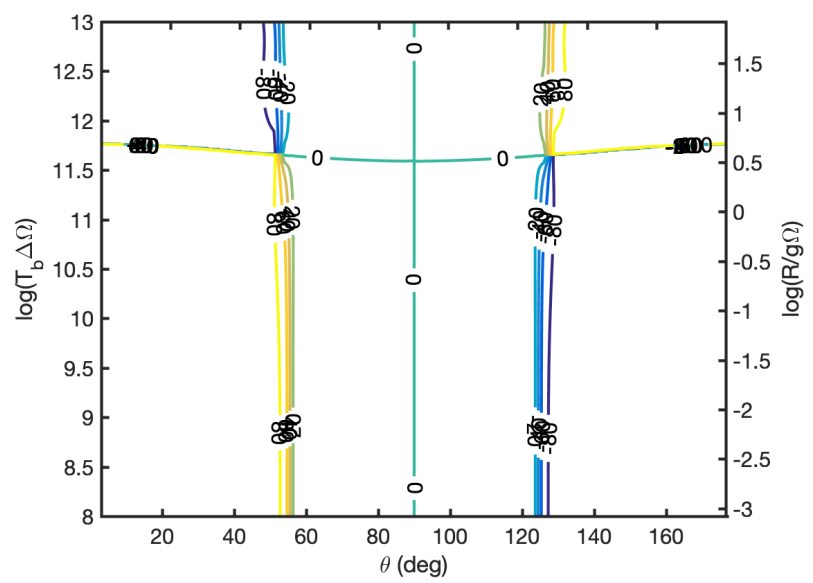

(b)

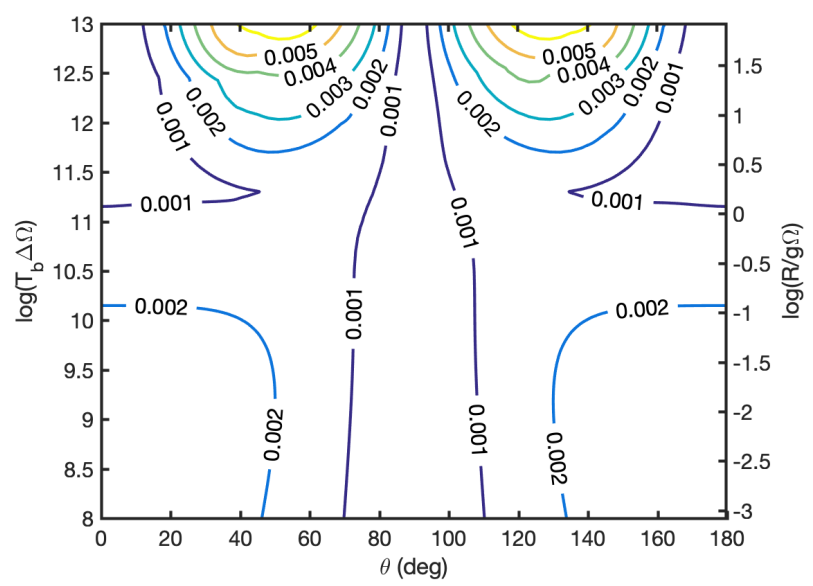

(c)

Fig. 8. Contour plots of the linear polarization fraction (a), angle (b) and circular polarization fraction (c) of water masers as a function of the propagation angle $\theta$ and the rate of stimulated emission. Magnetic field strength and thermal width denoted inside the figure. For simulations of other magnetic field strengths and thermal widths, see Figs. A.19 and A.20 in the Appendix.

simulation results for circular polarization depart from the LTE estimates. For the strongest masers, around $T_{b} \Delta \Omega \sim 10^{13} \mathrm{Ksr}$, we find (for $B=20 \mathrm{mG}$ ) the highest circular polarization, which can reach $0.55 \%$ around $\theta \sim 60^{\circ}$. Circular polarization in this region has only a minor dependence on the magnetic field strength and maser thermal width. 
We have already touched upon the complicating multitransitional nature of the water maser. It is very likely that asymmetries occur in the pumping of the different hyperfine transitions (see Walker 1984; Lankhaar et al. 2018). To further investigate this, we plot for a number of preferred hyperfine pumping ratios $\lambda=\lambda_{F=7-6} / \lambda_{\text {other }}$ the fractional circular and linear polarizations of a water maser at $\theta=45^{\circ}$ as a function of the maser luminosity. The $F=7-6$ transition is the strongest hyperfine transition and, incidentally, also the transition with the highest Zeeman coefficient. Figure 10, quite surprisingly, shows a negative correlation between the generated linear polarization and the favoring of the $F=7-6$ transition. However, the circular polarization increases as a result of the preferred pumping of the $F=7-6$ transition. Another interesting feature not apparent from the contour maps are the discontinuities in the linear and the circular polarization fractions. Discontinuities in these functions arise because of the complex nature of the multitransitional lines, and indeed do not occur for the most preferably pumped masers.

In Fig. 9, we present the $22 \mathrm{GHz}$ water maser spectra for different levels of saturation. It is immediately obvious that for all levels of saturation, the Stokes $I$ spectra are slightly asymmetric because of the multiple hyperfine components of this maser. This asymmetry is also seen in the linear polarization, which roughly follows the total intensity spectrum. We should note that circular polarization profiles are not the anti-symmetric S-shaped signals we observed for the single-transition $\mathrm{SiO}$ masers. Through the contributions from multiple hyperfine components an asymmetric circular polarization spectrum arises (Nedoluha \& Watson 1992; Vlemmings et al. 2001). A preferably pumped water maser, however, will show the characteristic S-shaped circular polarization signal.

\subsubsection{Polarized incident radiation}

We already observed in the $\mathrm{SiO}$ masers that for the higher angular momentum contours, the general structure of polarization contours is strongly influenced by the incoming polarized radiation. This is thus also the case for water masers, which generally also show weaker magnetic field interactions. The simulations with strongly polarized incoming radiation have nearly no general dependence on $\theta$ as the incoming linear polarization fraction smoothly deteriorates from $T_{b} \Delta \Omega>10^{12} \mathrm{Ksr}$. The weakly polarized incident radiation has a less pronounced effect on the polarization landscape, although it strongly dominates the landscape for $T_{b} \Delta \Omega<10^{10} \mathrm{Ksr}$.

These effects are also reflected in the landscape of circular polarization, which is strongly affected for the highly polarized incoming radiation, in contrast to the weak effects incident polarized seed radiation had on the $\mathrm{SiO}$ maser. Incident polarized seed radiation can cause relatively high fractions of circular polarization, especially in the region around $\theta=90^{\circ}$ (the region where isotropic incoming radiation leads to no circular polarization), where for the highly polarized incoming radiation the circular polarization can reach $5 \%$ (1\% for weakly polarized incoming radiation).

\subsubsection{Anisotropic pumping}

As a consequence of the shocked material that water masers occur in, photons that are associated with the radiative relaxation from the collisionally excited water molecules, may have a preferred escape direction. This can lead to a small anisotropy in the maser pumping. An analysis of our simulations of the anisotrop-

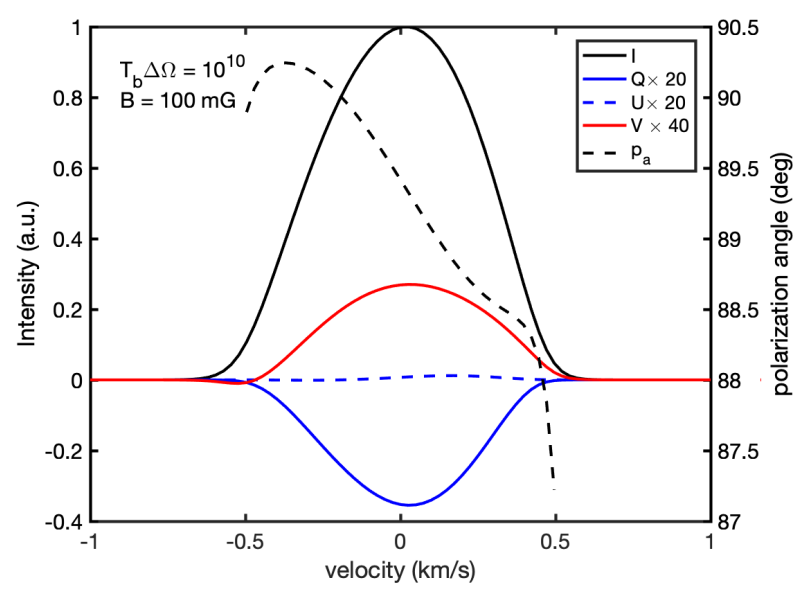

(a)

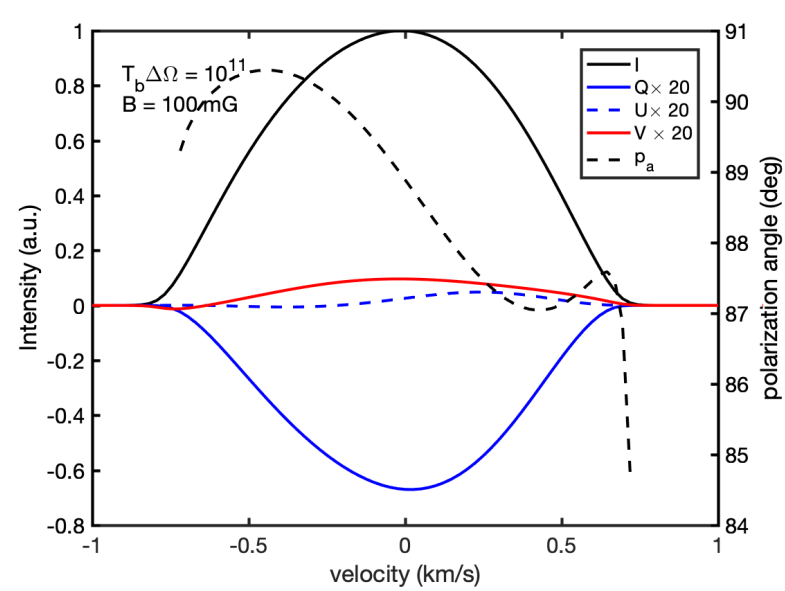

(b)

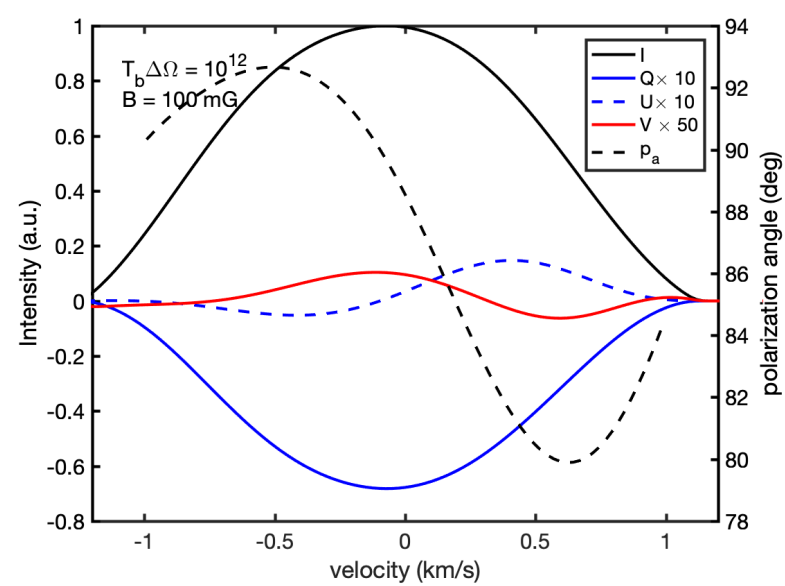

(c)

Fig. 9. Water $22 \mathrm{GHz}$ maser spectra for different levels of saturation. Plotted are all Stokes parameters (left y-axis) and polarization angles (right $y$-axis). The polarization angle is defined with respect to the magnetic field direction projected on the plane of the sky. Simulations were carried out at $B=100 \mathrm{mG}, v_{\text {th }}=1 \mathrm{~km} \mathrm{~s}^{-1}$, and with a magnetic field propagation angle of $\theta=45^{\circ}$.

ically pumped water maser showed that the linear accrual of polarization with the maser brightness is also characteristic of these masers. We note for the perpendicularly pumped water masers from Fig. 12 that masers of $\theta \rightarrow 90^{\circ}$ gather the most 


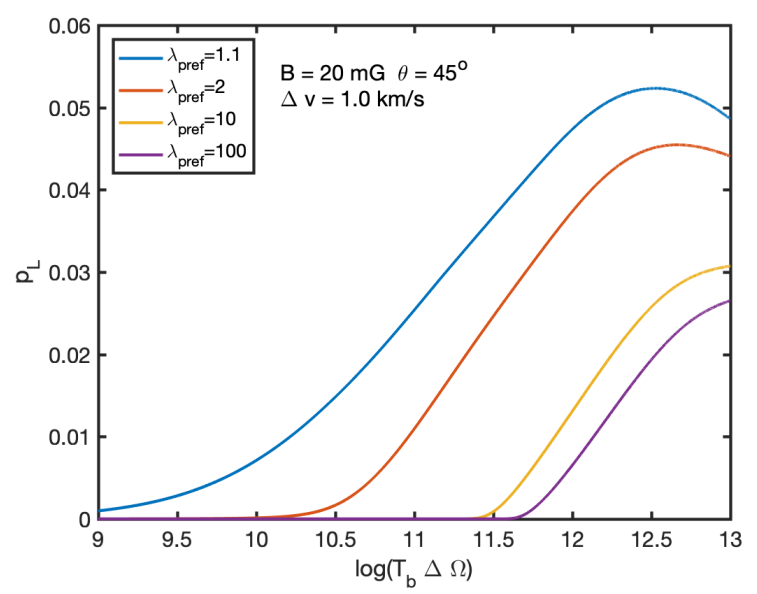

(a)

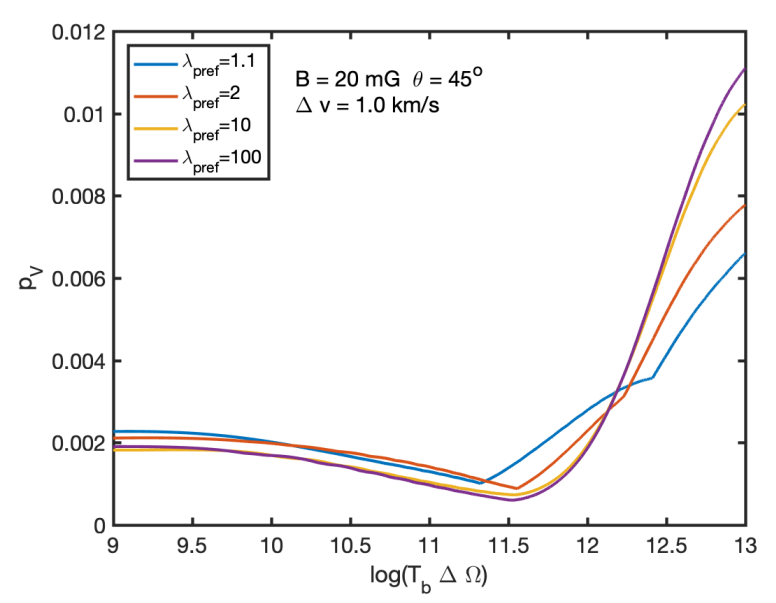

(b)

Fig. 10. Linear $(a)$ and circular polarization $(b)$ fraction of water masers as a function of the maser luminosity. Different degrees of preferred pumping are plotted. Magnetic field strength, angle $\theta$, and thermal width are indicated.

linear polarization from the propagation. For the water masers it seems that the standard magnetic field polarization mechanism has barely any effect on the polarization maps of both weak and strong anisotropy, as shown by the symmetry of the linear polarization landscapes. The polarization of these masers are almost independent of the magnetic field strength, but will be highly dependent on the intensity of the seed radiation, and on the anisotropy of the pumping $\eta$. Anisotropic pumping can generate arbitrary linear polarization fractions for the water masers.

High circular polarization fractions are only weakly associated with the drastically higher linear polarization from anisotropic pumping. Only the brightest of the strongly anisotropically pumped masers show significantly higher circular polarization, but not exceeding $5 \%$.

\section{Discussion}

We divide the discussion into two parts. First, we discuss the results we have presented in the previous section, and lay out the physical mechanisms behind the phenomena we observed from the simulations. In the second part of the discussion, we discuss these results in the context of previous $\mathrm{SiO}$ and water maser polarization observations.

\subsection{SiO masers}

\subsubsection{Simulations}

$90^{\circ}$ flip of the polarization angle. We observed two processes that can give rise to a $90^{\circ}$ flip in the polarization angle: an increase in rate of stimulated emission over two orders of magnitude, or the crossing of the magic angle $\theta_{\mathrm{m}}$. When $g \Omega \gtrsim 100 R$, the magnetic field determines the symmetry axis of the molecule. When this condition is fulfilled, and for the propagation radiation at an angle with the magnetic field smaller than $\theta_{\mathrm{m}}$, the polarization is oriented perpendicular to the magnetic field. For angles greater than $\theta_{\mathrm{m}}$, polarization is oriented parallel to the magnetic field. Thus, when we cross the magic angle and the condition $g \Omega \gtrsim 100 R$ is fulfilled, we see a sharp $90^{\circ}$ flip in the polarization angle across $\theta_{\mathrm{m}}$. For stronger masers, where $100 \gtrsim g \Omega / R \gtrsim 0$, we also observed a flip in the polarization angle, but this flip is gradual (over $\sim 10^{\circ}$ ), and does not predict zero polarization at the magic angle. The $90^{\circ}$ flip feature of $J=1-0 \mathrm{SiO}$ masers has recently be investigated by Tobin et al. (2019). Tobin et al. (2019) analyze the changing polarization fraction and angle of $\mathrm{SiO}$ maser spots across a clump. They assume a gradually changing propagation angle with the projected angular distance. From an analysis based on GKK73, they fit the observed polarization fraction and angle. A gradual $90^{\circ}$ flip is observed around the magic angle. According to their analysis, this is due to the free $K$ parameter that arises in the GKK73 models. Usually this parameter is assumed to be zero on the grounds of symmetry. According to our analysis, we do not need to invoke such a free parameter because, as we have seen in our simulations (Fig. 2), a blunt $90^{\circ}$ flip around the magic angle is characteristic of masers where the rate of stimulated emission is on the same order as the magnetic precession rate. Tobin et al. (2019) estimate $\log (R / g \Omega) \sim-1$, and our simulations of a magic angle flip at these conditions (Fig. $2, \log (R / g \Omega)=-1$ ) show a similar blunted magic angle flip in the polarization angle. We should note that our analysis underestimates the polarization fraction with respect to the observations, and non-Zeeman polarizing mechanisms need to be invoked to reach the observed polarization fractions.

Sometimes it is stated in the literature that in the limit $R \gg g \Omega$, maser polarization are randomly oriented (Plambeck et al. 2003). This is not the case. Even though the radiation field determines the alignment of the molecules, its interaction with the magnetic field through the maser medium is still the polarizing mechanism. It is therefore that the magnetic field determines the polarization direction. A $90^{\circ}$ flip across $\theta_{\mathrm{m}}$, however, will not occur in the case of $R \gg g \Omega$ as the orientation of the polarization is invariably parallel to the magnetic field. This is also associated with the alternative mechanism that leads to a $90^{\circ}$ polarization angle flip. When $R \ll g \Omega$ and the propagation angle is smaller than $\theta_{\mathrm{m}}$, the maser polarization will be oriented perpendicular to the magnetic field direction. However, if the rate of stimulated emission were to increase, or the magnetic field strength to decrease, and the condition $g \Omega \gg R$ were no longer fulfilled, the polarization would gradually align itself parallel to the magnetic field. A change of two orders of magnitude in $R$ or $g \Omega$ can cause a $90^{\circ}$ flip in the polarization angle.

A peak in polarization at $g \Omega \sim R$. Invariably, the highest linear and circular polarization fractions are observed for the case that the magnetic field strength is of the same order of magnitude as the rate of stimulated emission. This effect seems to be most pronounced for angles smaller than the magic angle, specifically around the propagation angle $\theta=20^{\circ}$. The extra polarization comes from a strongly enhanced Stokes $U$ component in 


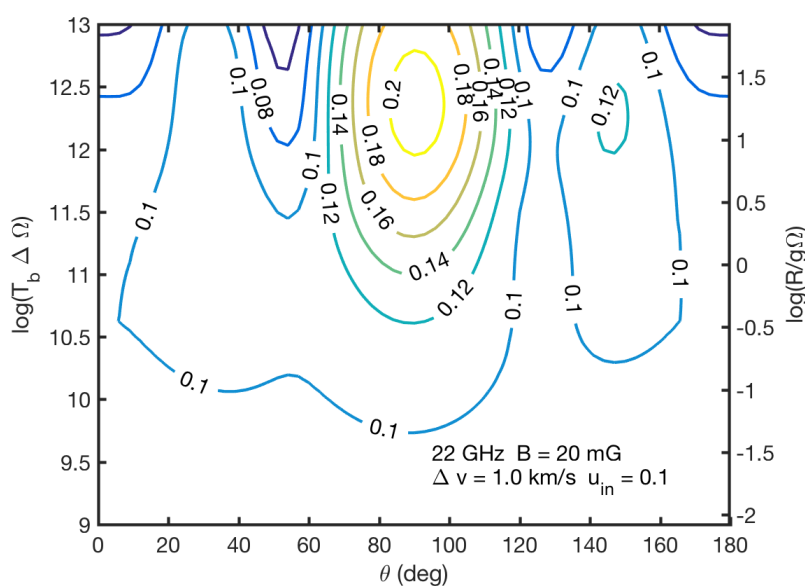

(a)

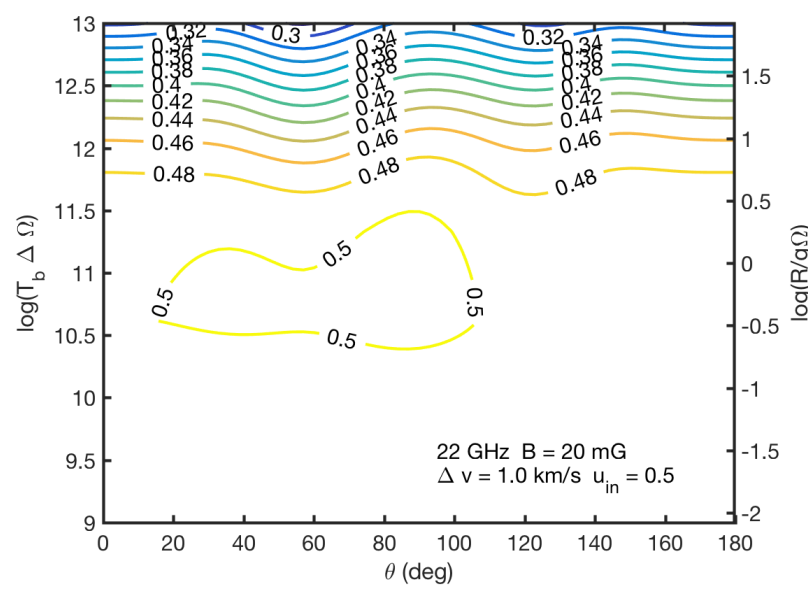

(c)

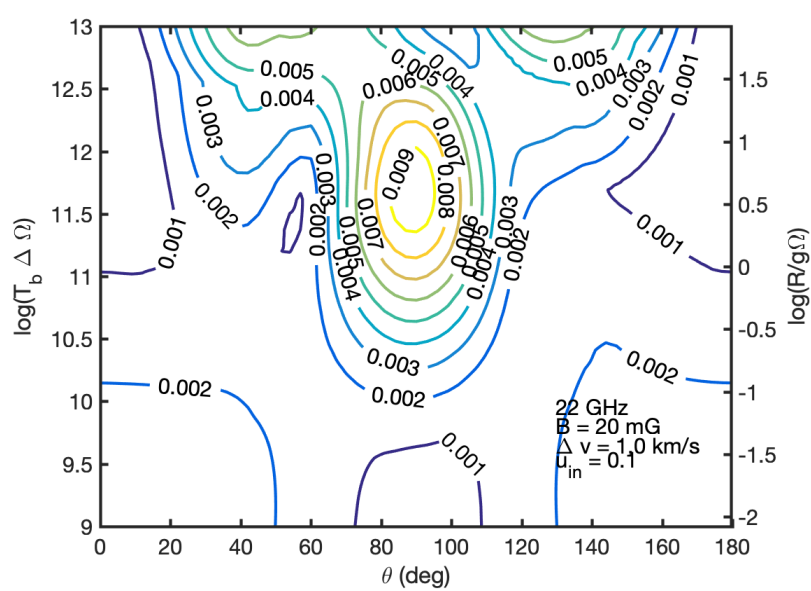

(b)

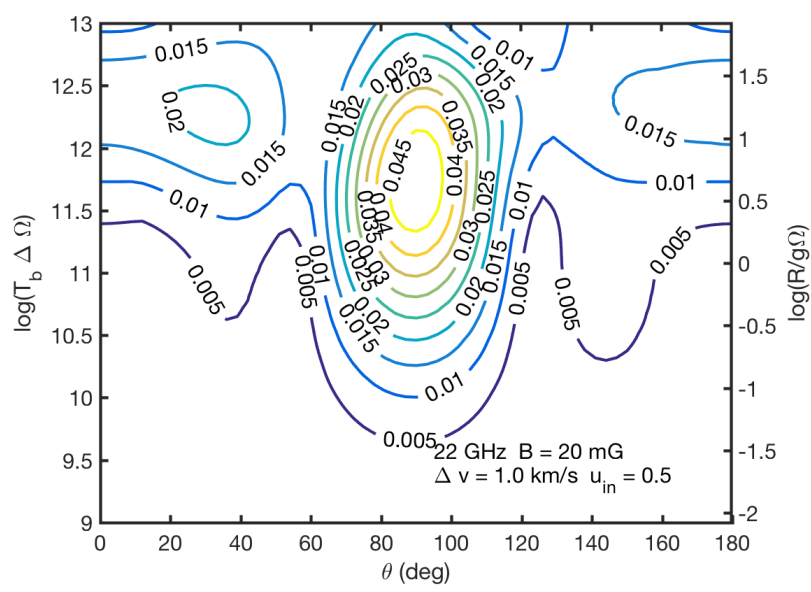

(d)

Fig. 11. Contour plots of the $(\mathrm{a}, \mathrm{c})$ linear and $(\mathrm{b}, \mathrm{d})$ circular polarization fraction of a water maser as a function of the propagation angle $\theta$ and the rate of maser luminosity. Maser simulations performed with incident polarized radiation of $(\mathrm{a}, \mathrm{b}) U / I=0.1$ and $(\mathrm{c}, \mathrm{d}) U / I=0.5$. Magnetic field strength and thermal width are indicated. For simulations with other $v_{\text {th }}$ and other magnetic field strengths, see Figs. A.21 and A.22 in the Appendix.

the radiation, and significant off-diagonal state density elements. The effect is absent for $90^{\circ}$ propagation because off-diagonal elements need not be invoked in these masers.

Absence of polarization below $R=1 \mathrm{~s}^{-1}\left(T_{b} \Delta \Omega=\right.$ $\left.9 \times 10^{6} \mathrm{Ksr}\right)$. Considering an isotropically pumped maser, and when $R$ is so small that $R \ll g \Omega$, we recognize from Eq. (7) that the radiation field has only a small influence on the populations of the magnetic substates of $\mathrm{SiO}$, and will be minimally polarized because of this. Also, because the (isotropic) decay of the states, described by the term $\Gamma$, is larger than $R$, the polarization of the states will be drastically lowered through the depolarizing decay.

The circular polarization of SiO masers. Just as for linear polarization, the highest circular polarization fraction was found in the region $R \sim g \Omega$. The polarization fraction in this region is not dependent on the maser thermal width. The high degree of circular polarization found here is due to an effect that was earlier described as "intensity-dependent circular polarization" (Nedoluha \& Watson 1994). Circular polarization is associated with the changing of the molecular symmetry axis that in the transition from $R<g \Omega$ to $R>g \Omega$ changes from parallel to the magnetic field, to parallel to the propagation direction.
A version of the above-described effect is also responsible for the circular polarization that will be generated by a randomly oriented magnetic field that is strong enough to align the molecule. Wiebe \& Watson (1998) investigated the propagation of polarized radiation through a medium with a randomly oriented magnetic field along $(128 \times 128)$ maser propagation paths. Along the path, linear polarization builds up. However, this linear polarization would not be aligned with the orientation of the molecules along the changing magnetic field. Locally, the linearly polarized radiation is rotated towards the local molecular alignment axis, with the associated production of circular polarization. In this way, relatively high degrees $(<3 \%)$ of circular polarization could be generated already from magnetic fields of $\sim 30 \mathrm{mG}$ (Wiebe \& Watson 1998). Because circular polarization is generated from the linear polarization, the circular polarization should not exceed a certain linear polarization-dependent limit. Through analyzing this relation, Cotton et al. (2011) found that the polarizing effects described by Wiebe \& Watson (1998) could not explain the high degrees of circular polarization found in their observations of $\mathrm{SiO} J=1-0$ masers. The circular polarization effects we have included in our models alone can also not fully explain the observations of Cotton et al. (2011) (see also our discussion of the maser line profiles below). 


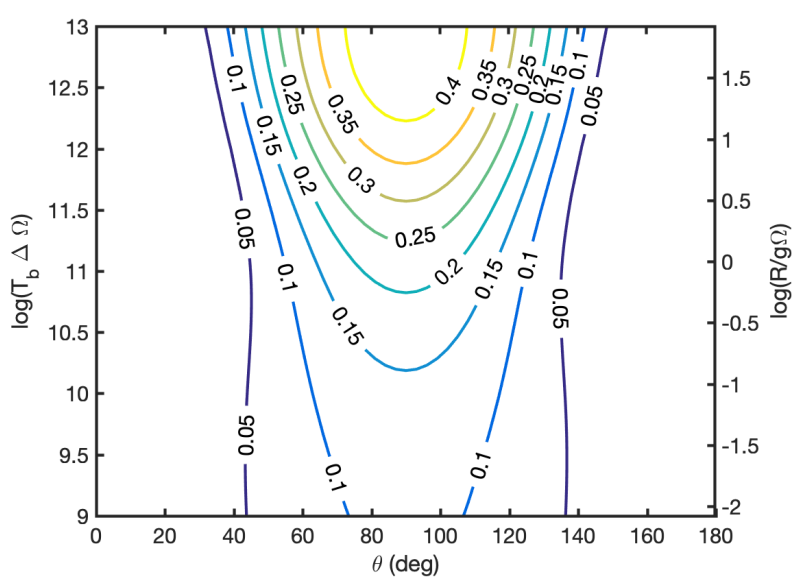

(a)

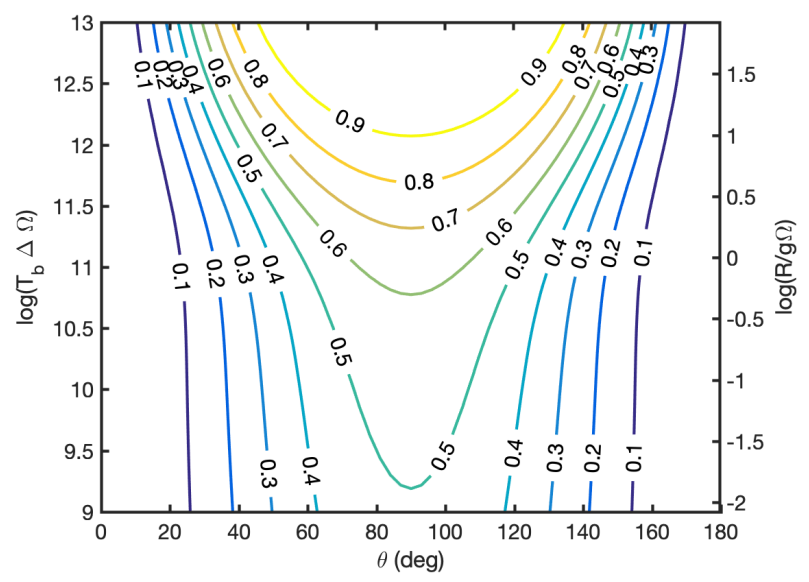

(c)

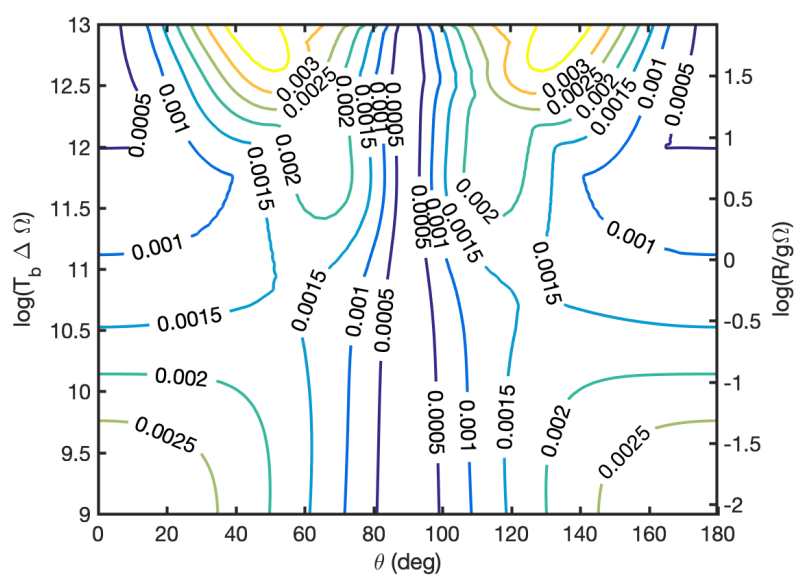

(b)

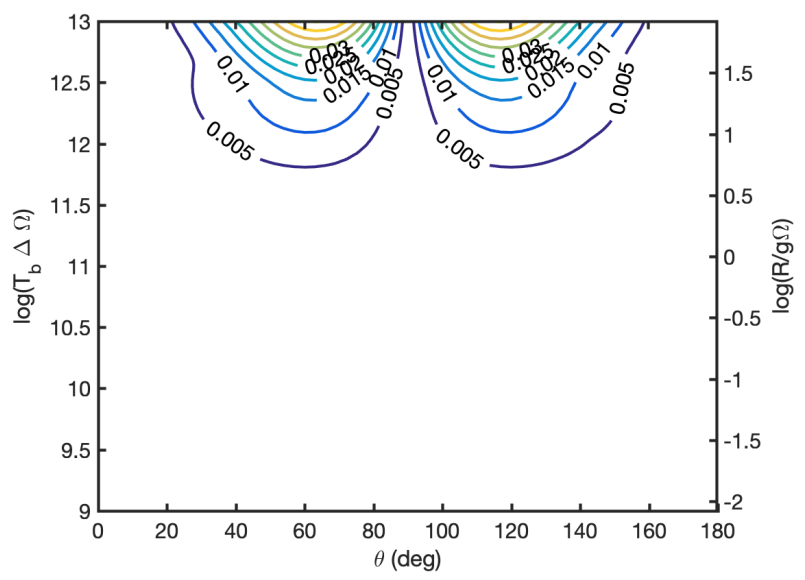

(d)

Fig. 12. Contour plots of the $(\mathrm{a}, \mathrm{c})$ linear and $(\mathrm{b}, \mathrm{d})$ circular polarization fraction of a water maser as a function of the propagation angle $\theta$ and the rate of maser luminosity. Maser simulations performed with anisotropic pumping perpendicular to both propagation and magnetic field direction, with anisotropy parameters of $(\mathrm{a}, \mathrm{b}) \eta=0.1$ and $(\mathrm{c}, \mathrm{d}) \eta=0.5$, and seed radiation of $T_{b}=0.1 \mathrm{~K}$. We used a magnetic field strength for these simulations of $B=20 \mathrm{mG}$ and thermal width $v_{\mathrm{th}}=1 \mathrm{~km} \mathrm{~s}^{-1}$.

Slow convergence to the GKK73 solutions. With a magnetic precession rate of $g \Omega=1500 \times B(\mathrm{G}) \mathrm{s}^{-1}$ and an isotropic decay rate of $\Gamma=5 \mathrm{~s}^{-1}$, the $\mathrm{SiO}$ maser generally fulfills the condition $g \Omega \gg \Gamma$. For the GKK73 solutions to maser linear polarization to apply, we furthermore have a constraint on the rate of stimulated emission such that $g \Omega \gg R \gg \Gamma$. For a value of $R$ in the range from $\Gamma$ to $g \Omega$, this requirement cannot be fulfilled for the magnetic field strengths expected around $\mathrm{SiO}$ masers. This is confirmed by our calculations, where we do not find the GKK73 solutions in the relevant parameter space. Convergence to the GKK73 solutions only occurs for unphysically strong magnetic fields and unphysically luminous masers.

Dependence of polarization on the angular momentum $J$ of the transition. The difference in polarization fraction between the $J=1-0$ and $J=2-1$ transitions is very large. For higher $J$ transitions, the polarization decrease with $J$ is less drastic. This phenomenon has already been observed by D\&W90 and N\&W90, and can be explained by the inability for the $J=0$ state to become polarized. The radiation field couples directly (in irreducible tensor terms) to the rank- 0,1 , and 2 elements. Coupling to higher rank elements is mediated by higher order effects, and is therefore orders of magnitude weaker. The maximum rank of the elements of a certain state is $2 J+1$. Therefore, all the polarization modes of the radiation field can couple directly to states of $J \geq 1$. Direct coupling of the polarization thus exists for all transitions but $J=1-0$, leading to this transition being highly polarized. The further consistent polarization decrease with $J$ can be explained by the introduction of higher rank irreducible population terms, whereto some of the polarization leaks away, but which do not couple directly to the radiation field.

Incident polarized seed radiation as a polarization mechanism. One principal result of the simulations with polarized seed radiation was contained in the distinction between a weak maser regime and a strong maser regime. We observed that the in the weak maser regime, the incident polarization was retained, and in the strong maser regime the polarization would converge to the polarization obtained with isotropic seed radiation. In the weak maser regime, the magnetic field defines the symmetry axis. Because the radiation field is so weak, it has no appreciable influence on the molecular states, and we can consider the states to be unpolarized. That means that amplification is characterized by a dominant $A_{\omega}$-term (see Eq. (14)). Thus, radiation is amplified and not altered in terms of polarization until it becomes a significant entity that can align and polarize the molecular states. After the weak maser regime, at about $\log (R / g \Omega)=-1$, a transition regime can be recognized where the initial polarization and the overall radiation have an appreciable influence on the 
molecular states. The feedback of the polarized molecular states in the propagation of the polarized radiation causes the radiation to converge to a polarization that is general for the system (in terms of $R, g \Omega$, and $\theta$ ), invariable of its initial conditions, which is what we call the strong maser regime. Convergence is attained later for strongly polarized seed radiation, and lower magnetic fields. High degrees of polarization can be obtained in the transition regime. Later in this discussion, we comment on the effect these high degrees of linear polarization have on the circular polarization.

Anisotropic pumping as a polarization mechanism. For the anisotropically pumped maser we have a weak maser regime and a strong maser regime as well. We should note, however, that these regimes carry a different meaning with respect to the regimes of the masers with polarized seed radiation of the same name. The weak maser regime of the anisotropically pumped maser is characterized by a linear growth of the polarization with maser luminosity. This growth can continue to arbitrary degrees of linear polarization until the radiation becomes strong enough to align the molecular states. In the weak maser regime, because the pumping is anisotropic (where the anisotropic part can be represented by a second-rank irreducible tensor), polarization is pumped into the molecular states causing a feed to the radiation field via the propagation coefficients $B_{\omega}$ and $F_{\omega}$ (see Eq. (14)). The buildup of polarization is thus dependent on the relative anisotropy in the pumping $\epsilon$, but also on the relative size of $A_{\omega}$, given by $\delta$ (Eq. (18)), leading to the anisotropy parameter $\eta=\epsilon / \delta$. The buildup of polarization in the weak maser regime is independent of the magnetic field and is not associated with circular polarization, but it is dependent on the brightness of the seed radiation.

When radiative interactions become strong enough to influence the alignment of the molecule, a transition regime begins and, generally, the strongly polarized radiation begins to lose most of its polarization. The alignment of the molecular states counters the large overshoot in polarization left from the weak maser regime, and converges in the strong maser regime to a polarization that is a function of the anisotropy of the pumping (including direction), $R$ and $\theta$, which is independent of the incoming radiation.

Maser line profiles. Maser line profiles are often much narrower than their LTE counterparts because of the stimulated emission mechanism. This is most obvious when the rate of stimulated emission is near the isotropic decay rate $R \sim \Gamma$. After that point, broadening of the line starts and increases with $R$. From analyzing the polarized spectra we observe that linear polarization spectra roughly follow the Stokes $I$ spectrum, which is expected because the molecular states become polarized by the directional intensity field. The difference in polarizing intensity also leads to a variable polarization angle across the spectrum. This is particularly present for rates of stimulated emission $R \sim g \Omega$. The degree of change of the polarization angle across the maser line can therefore be taken as a proxy for the saturation level.

We observe that the polarizing mechanism under investigation in our simulations produce perfect anti-symmetrical S-shaped spectra for the Stokes $V$ component of the radiation field. Such anti-symmetric spectra are often seen in astrophysical maser spectra (Amiri et al. 2012). However, asymmetric Stokes $V$ spectra are observed regularly as well. Cotton et al. (2011) report the observation of many strongly asymmetrically circularly polarized $\mathrm{SiO}$ masers. Our models do not produce such asymmetrical spectra in the absence of hyperfine multiplicity, but would need to include alternative effects. A velocity gradient across the maser column or the presence of strong anisotropic resonant scattering in either a foreground cloud or as a part of the maser action itself are known to be able to produce asymmetric Stokes $V$ spectra (Houde 2014). Kinematic effects coming from other polarized background maser sources could also explain the asymmetric signals.

Interesting evidence for kinematic effects can be found by analyzing some individual maser line spectra (Cotton et al. 2011). The polarization spectra of the maser spot in Fig. 5, row 1, from Cotton et al. (2011), show a similar variation for the polarization angle across the spectrum to that in our Fig. 3 of the spectral polarization of $\mathrm{SiO}$ masers. This maser shows an $\mathrm{S}$-shaped anti-symmetric Stokes $V$ spectrum. Analyzing then rows 3 and 4 of the same figure in Cotton et al. (2011), we see a variation in the polarization angle across the maser line that is more reminiscent of the $22 \mathrm{GHz}$ water maser spectra of Fig. 9. The circular polarization of these signals is also similar to our spectral models of the water masers (Fig. 9). The different hyperfine components in water masers can reasonably be considered to emulate kinematic effects as they would occur for an $\mathrm{SiO}$ maser. A deeper analysis of such effects is beyond the scope of this paper, but we can suggest that asymmetric circular polarization signals can be the product of kinematic effects.

Alternative polarizing mechanisms and circular polarization. An interesting result of our investigations into the effects of anisotropic pumping and polarized incident radiation is the rather marginal effects these polarizing mechanisms have on the circular polarization fraction of the maser. This can be best explained in a tensorial picture of the matter-radiation interactions. In a tensorial picture of the polarized radiation, Stokes $Q$ and $U$ (and I) are expressed as second-rank components of the irreducible radiation tensor, while Stokes $V$ is a first rank component of this tensor (Degl'Innocenti \& Landolfi 2006). Direct polarization of the molecular states by linearly polarized radiation thus only affects the second-rank populations. It is also the secondrank populations that are pumped by the anisotropic pumping. Thus, for incident polarized radiation and anisotropic pumping, there is no direct coupling to the first rank populations, and thus no direct coupling to the Stokes $V$ radiation. The Stokes $V$ will only be slightly enhanced by higher order effects, such as anisotropic resonant scattering (Houde et al. 2013), which will be more pronounced with high linear polarization of the radiation.

Observational heuristics. Generally, we can recognize different regimes that are connected to the maser luminosity that show particular behavior regarding maser polarization. We therefore define characteristic maser luminosities that will simplify the analysis. The maser luminosity at which the rate of stimulated emission is equal to the rate of magnetic precession is defined as

$\left(T_{b} \Delta \Omega\right)_{\text {mag. sat. }}=\frac{4 \pi \omega_{0}(g \Omega)}{A_{i j} k_{\mathrm{B}}}$,

where $\omega_{0}$ is the maser's natural frequency, $k_{\mathrm{B}}$ is the Boltzmann constant, and $A_{i j}$ is the Einstein coefficient. Furthermore, we define the luminosity after which the maser will start broadening because of saturation:

$\left(T_{b} \Delta \Omega\right)_{\text {sat. }}=\frac{4 \pi \omega_{0} \Gamma}{A_{i j} k_{\mathrm{B}}}$.

Table 3 gives these luminosities for the different $\mathrm{SiO}$ masers. Already at weak magnetic fields of $B>10 \mathrm{mG},\left(T_{b} \Delta \Omega\right)_{\text {mag. sat. }}>$ 
Table 3. Characteristic maser luminosities temperatures for $v=1 \mathrm{SiO}$ masers.

\begin{tabular}{lcc}
\hline \hline Transition & $\left(T_{b} \Delta \Omega\right)_{\text {sat }}(\mathrm{Ksr})$ & $\left(T_{b} \Delta \Omega\right)_{\text {mag. sat. }} / B\left(\mathrm{Ksr} \mathrm{mG}^{-1}\right)$ \\
\hline$J=1-0$ & $4.35 \times 10^{7}$ & $6.52 \times 10^{6}$ \\
$J=2-1$ & $9.00 \times 10^{6}$ & $1.35 \times 10^{6}$ \\
$J=3-2$ & $3.73 \times 10^{6}$ & $5.60 \times 10^{5}$ \\
$J=4-3$ & $2.03 \times 10^{6}$ & $3.04 \times 10^{5}$ \\
$J=5-4$ & $1.27 \times 10^{6}$ & $1.90 \times 10^{5}$ \\
\hline
\end{tabular}

$\left(T_{b} \Delta \Omega\right)_{\text {sat. }}$. For the weakest masers, where $T_{b} \Delta \Omega<\left(T_{b} \Delta \Omega\right)_{\text {sat }}$, linear polarization is mostly absent in the emission because of the depolarizing effect of the isotropic decay. Circular polarization is generated through the Zeeman effect. Because of the Zeeman effect, the $\sigma^{ \pm}(\Delta m= \pm 1)$ transitions have a slight spectral disposition, which, if subtracted from each other, yield the $\mathrm{S}$-shaped Stokes $V$ spectrum. It can be shown via a LTE analysis that the circular polarization follows (Fiebig \& Güsten 1989; Watson \& Wyld 2001)

$p_{\mathrm{V}}=\frac{2 A_{J J^{\prime}} B_{\mathrm{Gauss}} \cos \theta}{\Delta v_{\mathrm{L}}(\mathrm{km} / \mathrm{s})}$,

where $A_{J J^{\prime}}$ is a transition-dependent constant and $\Delta v_{\mathrm{L}}$ is the FWHM of the maser profile. The LTE estimates for the constant $A_{J J^{\prime}}$ of $\mathrm{SiO}$ transitions are

$A_{J J^{\prime}}=\frac{1.1807 \times 10^{-3}}{J}$,

where $J$ is the rotational quantum number of the upper state. It is usual to employ a LTE analysis of the circular polarization of weak masers since the maser circular polarization mechanism for these masers is similar to the LTE mechanism. To check the validity of this analysis, we plot the results of our simulations for the $A_{J J^{\prime}}$ constants for three transitions at $B=1 \mathrm{G}$ in Fig. 13. For $T_{b} \Delta \Omega \lesssim\left(T_{b} \Delta \Omega\right)_{\text {mag. sat }} / 1000$, the $A_{J J^{\prime}}$ coefficient obtained from our simulations is similar to the LTE estimate. However, already for $T_{b} \Delta \Omega \sim\left(T_{b} \Delta \Omega\right)_{\text {mag. sat }} / 100$, we find that the $A_{J J^{\prime}}$ constants from our simulations are twice that of the LTE estimate, meaning that a LTE analysis of the magnetic field strength would lead to an overestimation by a factor of 2 .

For masers $T_{b} \Delta \Omega \ll\left(T_{b} \Delta \Omega\right)_{\text {mag. sat. }}$, the highest circular polarization is found for the masers that have not started broadening yet $\left(T_{b} \Delta \Omega \sim\left(T_{b} \Delta \Omega\right)_{\text {sat }}\right)$. After $T_{b} \Delta \Omega>\left(T_{b} \Delta \Omega\right)_{\text {sat }}$, the maser starts saturating with the associated broadening. As long as the magnetic precession rate remains far greater than the rate of stimulated emission, $T_{b} \Omega \ll\left(T_{b} \Delta \Omega\right)_{\text {mag. sat. }}$, the circular polarization will decrease because of this broadening. Linear polarization starts to build up, oriented parallel $\left(\theta>\theta_{\mathrm{m}}\right)$ or perpendicular $\left(\theta<\theta_{\mathrm{m}}\right)$ to the projected magnetic field direction. Linear polarization rises steadily with the maser luminosity until it reaches the GKK73 solution for the specific propagation angle. However, long before the GKK73 solution is reached, when the maser luminosity approaches $\left(T_{b} \Delta \Omega\right)_{\text {mag. sat. }}$, alternative polarization effects will take over.

In the regime of $T_{b} \Delta \Omega \sim\left(T_{b} \Delta \Omega\right)_{\text {mag. sat. }}$, polarization associated with the change in molecular alignment manifests itself in the emission spectrum. Linear polarization in this regime can therefore exceed the GKK73 solutions by $\sim 10 \%$. For $\theta<\theta_{\mathrm{m}}$, the polarization vector changes from perpendicular to parallel between $T_{b} \Delta \Omega \sim\left(T_{b} \Delta \Omega\right)_{\text {mag. sat. }} / 10$ and $T_{b} \Delta \Omega \sim 10\left(T_{b} \Delta \Omega\right)_{\text {mag. sat. }}$, and has intermediate polarization

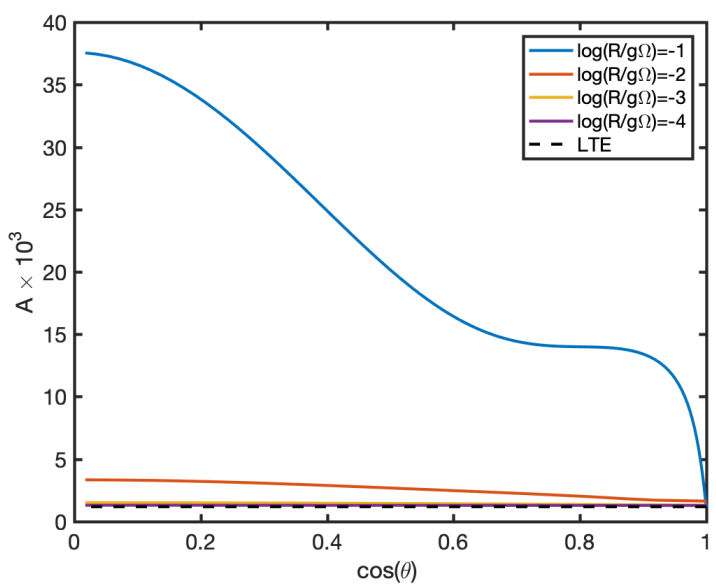

(a)

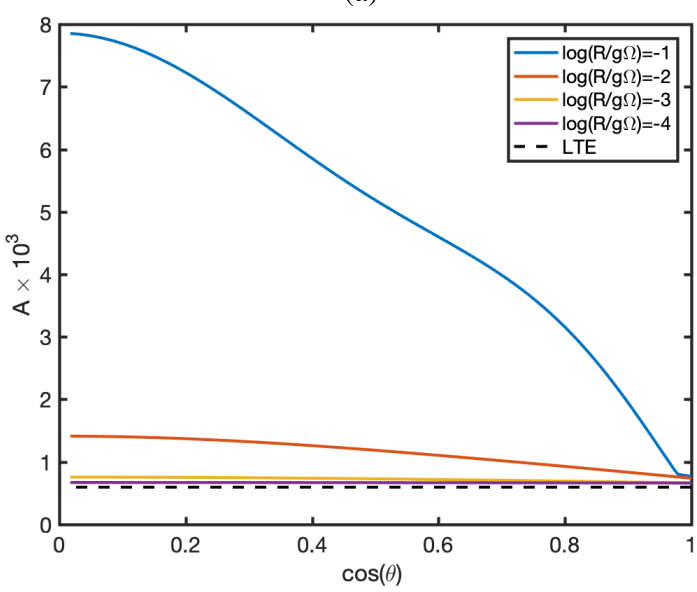

(b)

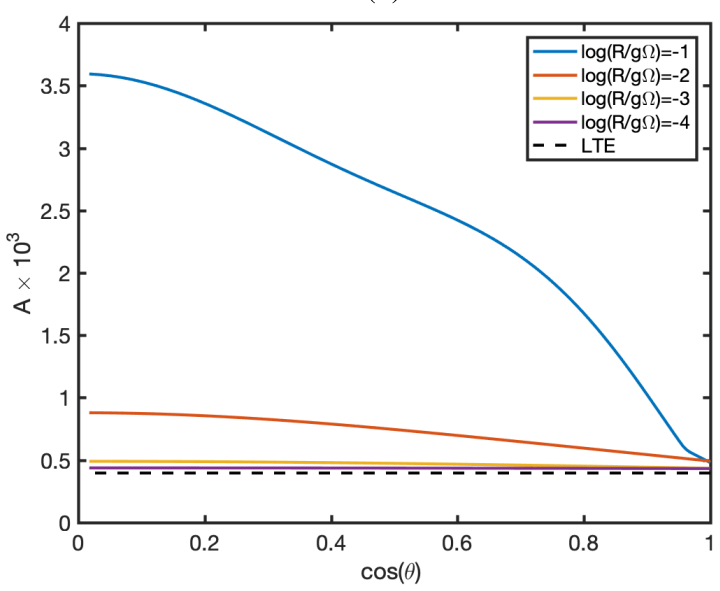

(c)

Fig. 13. The $A_{J J^{\prime}}$ coefficients of an isotropically pumped $\mathrm{SiO}$ maser at $B=1 \mathrm{G}$ as a function of the magnetic field propagation direction angle $\cos \theta$. The different panels give the (a) $J=1-0$, (b) $J=2-1$, and (c) $J=3-2$ transitions. Plots are given for different $\log (R / g \Omega)$. The LTE solutions (constant over $\cos \theta$ ) are indicated with a dotted line.

angles within this range. With the gradual changing of the polarization angle a lot of circular polarization is associated. This is reflected in the high $A_{J J^{\prime}}$ constants for the circular polarization (see Fig. 13). Constancy of $A_{J J^{\prime}}$ over $\theta$ is also lost. For the lower angular momentum transitions, there is a large overshoot of the Zeeman circular polarization. Already for weak magnetic fields, high degrees of circular polarization can be generated 
and the Zeeman analysis cannot be applied directly. Extraction of the magnetic field strength from masers in the regime $T_{b} \Delta \Omega \sim\left(T_{b} \Delta \Omega\right)_{\text {mag. sat. }}$ can be achieved by a simultaneous analysis of both the linear and circular polarization of the radiation, which is demonstrated later on.

Alternative polarizing mechanisms such as anisotropic pumping can enhance the polarization of masers to arbitrarily high degrees. The presence of anisotropic pumping could be determined by analyzing the weaker masers $\left(T_{b} \Delta \Omega \ll\right.$ $\left.\left(T_{b} \Delta \Omega\right)_{\text {mag. sat. }}\right)$ for their polarization. The linear polarization degree of these masers should be proportional to their luminosity. When the anisotropically pumped maser approaches the luminosity $\left(T_{b} \Delta \Omega\right)_{\text {mag. sat. }}$, linear polarization drops as the standard polarizing mechanisms take over. Richter et al. (2016) find in their VLBA observations of VY CMa the strongest polarization for the weakest masers, and observe a drop in polarization after a certain maser luminosity threshold. Turning to polarized seed radiation, in the regime $\left(T_{b} \Delta \Omega \ll\left(T_{b} \Delta \Omega\right)_{\text {mag. sat. }}\right)$, the polarization is simply that of the seed radiation, and has no dependence on the maser luminosity. Circular polarization is only slightly enhanced for alternatively polarized masers.

Finally, it should be noted that the polarization properties are a function of the maser luminosity $T_{b} \Delta \Omega(\propto R)$, which cannot be measured directly. To estimate the maser luminosity from observations requires knowledge of the maser beaming solid angle $\Delta \Omega$. Direct observations of $\Delta \Omega$ have proven difficult to date, but have been performed with VLBA measurements to $\mathrm{SiO}$ around AGB stars (Assaf et al. 2013). In these observations, Assaf et al. (2013) measure, with a sizable error margin due to relatively low resolution, $\Delta \Omega \sim 5 \times 10^{-2}$ sr. This maser beaming solid angle is independent of its brightness when the amplification is matterbounded (most easily approximated by the cylindrical maser) (Elitzur et al. 1992). When the maser is amplification-bounded (most easily approximated by the spherical maser) the beaming solid angle drops with increasing maser brightness. To the best of our knowledge, no investigations have been done on the geometrical nature of the maser amplification of $\mathrm{SiO}$ masers.

\subsubsection{SiO maser polarization observations}

Many SiO maser polarization observations have been performed. Very-long-baseline interferometry (VLBI) observations have shown that $\mathrm{SiO}$ masers orient themselves in a ring-like structure around the central stellar object. The polarization of these $\mathrm{SiO}$ masers, irrespective of their angular momentum transition, show well-ordered polarization vectors with respect to this structure (Kemball \& Diamond 1997; Cotton et al. 2004; Plambeck et al. 2003; Vlemmings et al. 2011b, 2017). This is taken to be an indicator of an ordered magnetic field. The linear polarization fraction of individual masers can be arbitrarily high, but median values are much lower. The $J=1-0$-transition has median linear polarization fractions of $\sim 25 \%$ (Kemball $\&$ Diamond 1997). Analyzing the angular momentum dependence of the linear polarization fraction, we note the general trend of lower degrees of polarization for the higher angular momentum transitions. This is not to say that high fractions $(>50 \%)$ of linear polarization do not occur for high-J SiO maser transitions. It is almost certain that the most strongly polarized masers are the product of anisotropically pumped maser action as incident polarized radiation at these fractions is unlikely and should lead to the same effect for the high- $J$ masers. The hypothesis of anisotropic pumping could be further supported by correlating maser brightness for the weaker masers $(R<g \Omega)$ to linear polarization.
The relationship between maser brightness and polarization fraction is unfortunately not well-documented. However, Barvainis et al. (1987) meticulously tabulated their observations, from which we could construct a scatter plot that indicated the lowest fractions of polarization for the strongest masers. This is in line with the simulations we delineate above, where we see that above $R \sim g \Omega$, polarization fractions start to drop.

Herpin et al. (2006) were able to derive an interesting relation between the circular polarization and linear polarization of $\mathrm{SiO} J=2-1$ masers. In a large survey of a number of evolved stars, they analyzed, among other things, the correlation between linear and circular polarization fractions of the $\mathrm{SiO}$ masers. Even though the correlation was highly scattered, a clear linear relation was observed between linear and circular polarization (Fig. 4, Herpin et al. (2006)). Also invariably, high circular polarization was associated with high linear polarization. To simulate their observations, we used CHAMP to compute the linear and circular polarization fractions of 200 isotropically pumped $\mathrm{SiO}$ masers at randomly selected luminosities between $T_{b} \Delta \Omega=10^{6}-10^{11} \mathrm{Ksr}$ and randomly selected propagation angles $\theta$. We plot the results for $\mathrm{SiO} J=2-1$ masers pumped at $T=1000 \mathrm{~K}$, and magnetic field of $B=1 \mathrm{G}$ in Fig. 14 . Herpin et al. (2006) found a rough linear relation between the linear and circular polarization, $p_{\mathrm{V}}=0.25 p_{\mathrm{L}}+0.015$, which we plot in the figure.

Only for lower degrees of linear polarization do we find a reasonable agreement between our simulations and the observations of Herpin et al. (2006). Our simulations seem to underestimate the circular polarization with respect to the observations of Herpin et al. (2006). This is especially true for the strongly linearly polarized masers. One factor that could play a role here is the enhancement of circular polarization by the presence of a velocity gradient along the propagation path of the SiOmaser. N\&W94 have shown that this can enhance the circular polarization. Another explanation of the high circular polarization might be the anisotropic resonant scattering of maser radiation by a foreground cloud of non-masing $\mathrm{SiO}$ (Houde et al. 2013; Houde 2014). Via anisotropic resonant scattering, linearly polarized radiation can be converted to circularly polarized radiation. Anisotropic resonant scattering does not necessarily produce the anti-symmetric S-shaped Stokes $V$ spectrum profile characteristic of circular polarization generated by the Zeeman effect, but it can arise from scattering of a cloud outside the velocity range of the maser. Non-anti-symmetric Stokes $V$ spectra were observed by Herpin et al. (2006), but they can also be explained by a velocity gradient along the propagation path of the maser, or the lack of spatial resolution from the single-dish observations.

\section{2. $\mathrm{H}_{2} \mathrm{O}$ masers}

\subsubsection{Simulations}

The relevant characteristic maser luminosities are given in Table 4. We list the relevant luminosities for individual hyperfine transitions as well as the blended line. Compared to the $\mathrm{SiO}$ maser, radiative interactions remain relatively weak with respect to magnetic interactions up to high maser luminosities. This is due to the much smaller line strength of this maser transition, which means that the Zeeman effect will be the dominating polarizing mechanism up to high maser luminosities, and will thus follow Eq. (38) up to high maser brightness. Linear polarization will also remain rather low because the isotropic decay will be a dominant de-polarizing entity up to $\left(T_{b} \Delta \Omega\right)_{\text {sat }}(\mathrm{Ksr})$ at 


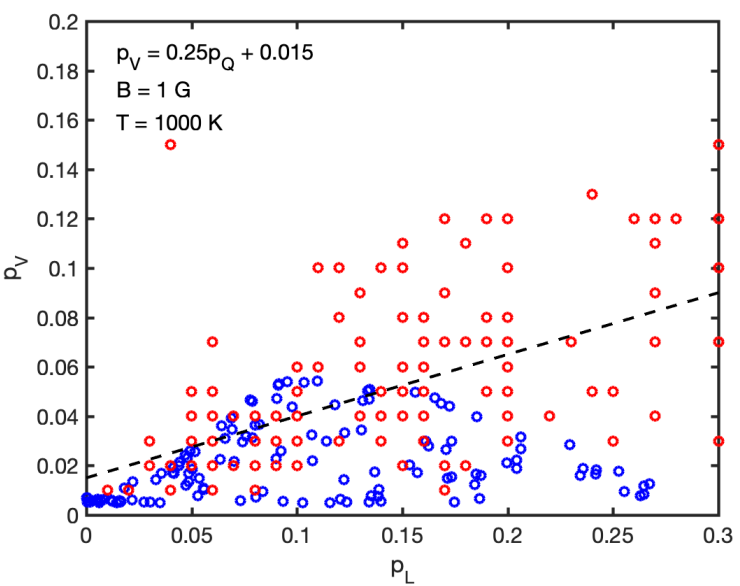

(a)

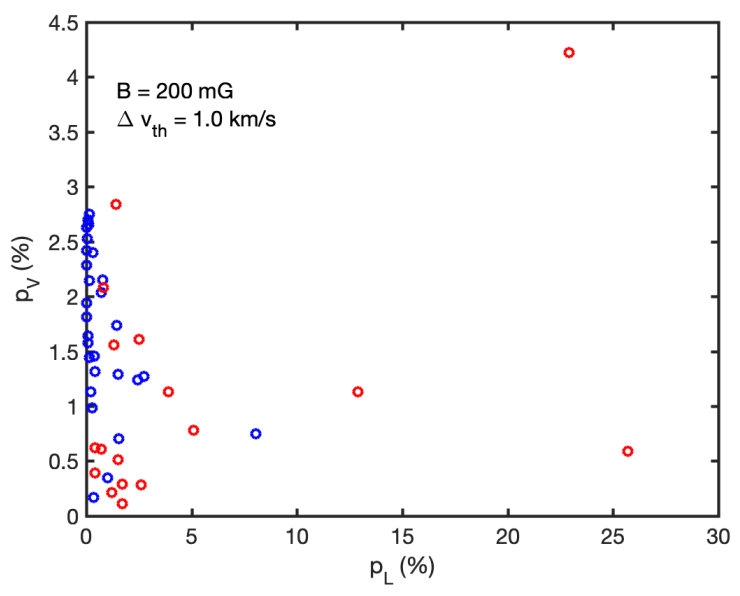

(b)

Fig. 14. Scatter plot for the linear-to-circular polarization fraction relation $p_{\mathrm{L}}-p_{\mathrm{V}}$. In red, the observations of (a) Herpin et al. (2006) and (b) Surcis et al. (2011) are reported. In panel (a) the blue points come from our simulations of the $J=2-1$ transition at $B=1 \mathrm{G}$, with the kinetic temperature of the maser $T=1000 \mathrm{~K}$. In (panel $b$ ) the blue points come from our simulations of the isotropically pumped water maser at $v_{\text {th }}=1.0 \mathrm{~km} \mathrm{~s}^{-1}$ and $B=200 \mathrm{mG}$. To generate these scatters, we computed the polarization fractions from (a) 200 (b: 30) isotropically pumped masers with a randomly selected a luminosity between (a) $T_{b} \Delta \Omega=10^{6}-10^{11}$ (b: $T_{b} \Delta \Omega=10^{8.5}-10^{11}$ ) and a randomly selected propagation angle $\theta$. In the scatter plot, we do not include masers that show polarization $<0.5 \%$ (b: $<0.1 \%$ ). In (panel $a$ ), we also report the linear regression analysis result from Herpin et al. (2006).

about $\sim 10^{10}$ Ksr. Strong linear polarization is thus only seen for the strongest masers.

For the regime $T_{b} \Delta \Omega \ll\left(T_{b} \Delta \Omega\right)_{\text {mag. sat }}$, the maser circular polarization can be described by Eq. (38). A LTE analysis of the constant $A_{F F^{\prime}}$, gives for the individual hyperfine transitions, $A_{76}=13.3, A_{65}=8.3$, and $A_{54}=1.0$. A LTE analysis of a completely blended water maser line gives $A_{\text {blend }}=8.2$. Figure 15 shows the results of our full radiative transfer analysis of the circular polarization constants. Apart from the standard maser line profile, water masers are further broadened by their hyperfine structure. This leads to an overestimation of $\Delta v_{\mathrm{L}}$. The dominant Zeeman effect though, comes from a single hyperfine transition. This produces higher Zeeman $A_{F F^{\prime}}$ coefficients with respect to a LTE analysis. We observe that this effect is most pronounced for masers pumped at $v_{\text {th }}=0.6 \mathrm{~km} \mathrm{~s}^{-1}$, where the hyperfine tran-
Table 4. Characteristic maser luminosities for the $22 \mathrm{GHz}$ water maser.

\begin{tabular}{lcc}
\hline \hline Transition & $\left(T_{b} \Delta \Omega\right)_{\text {sat }}(\mathrm{Ksr})$ & $\left(T_{b} \Delta \Omega\right)_{\text {mag. sat. }} / B\left(\mathrm{Ksr} \mathrm{mG}^{-1}\right)$ \\
\hline$F=7-6$ & $7.2 \times 10^{9}$ & $3.1 \times 10^{10}$ \\
$F=6-5$ & $7.4 \times 10^{9}$ & $2.0 \times 10^{10}$ \\
$F=5-4$ & $7.5 \times 10^{9}$ & $2.3 \times 10^{9}$ \\
blend & $7.4 \times 10^{9}$ & $6.0 \times 10^{9}$ \\
\hline
\end{tabular}

sitions are minimally mixed. At $v_{\text {th }}=2.0 \mathrm{~km} \mathrm{~s}^{-1}$, the hyperfine broadening is negligible and the LTE value for the $A_{F F^{\prime}}$ coefficient of the $F=7-6$ hyperfine transition is returned for the weakest masers.

Paradoxically, the preferred pumping of the hyperfine component with the strongest Zeeman effect has a consequence that the linear polarization deteriorates in the relevant intensity window for water masers (up to $T \Delta \Omega=10^{13}$ ). This is due to the fact that the magnetic field precession rate effectively increases, and the $\left(T_{b} \Delta \Omega\right)_{\text {mag. sat. }}$ is out of reach. Thus, the change in molecular symmetry-axis that is associated with the production of linear polarization occurs only for the strongest masers. Therefore, transitions with weaker Zeeman interactions are associated with higher degrees of linear polarization in the relevant brightness regime for the water maser. We should note that this effect is not as pronounced for the high-temperature masers, where the broadening of the lines causes the other transitions to blend in more. The maser circular polarization is proportional to the strength of the Zeeman effect, as expected from Eq. (38).

\subsubsection{Water maser polarization observations}

Richards et al. (2011) performed water maser observations around AGB stars with e-MERLIN. For the brightest masers, a beaming solid angle on the order of $\Delta \Omega \sim 1.5 \times 10^{-3} \mathrm{sr}$ was found. For some AGB stars, the geometrical masing mechanism seemed to be amplification-bounded, but hints of matter-bounded amplification were also found for some sources. Line profile analysis by Vlemmings \& van Langevelde (2005) revealed a $\Delta \Omega \sim 10^{-2}-10^{-3}$ sr for water masers around AGB stars. A line profile analysis of the extremely strong water masers around Orion-KL, yielded beaming solid angles as low as $\Delta \Omega \sim$ $10^{-5} \mathrm{sr}$ (Nedoluha \& Watson 1991).

Water masers have been observed for their polarization on many occasions around evolved stars (Vlemmings et al. 2006a) and around star-forming regions (Garay et al. 1989). The most striking observations were the early observations of the flaring, very strong "super" water maser (Garay et al. 1989; Fiebig \& Güsten 1989). Garay et al. (1989) report the seven-year monitoring of the polarization characteristics of the most powerful water maser feature of Orion-KL. Brightness temperatures over $T_{b}=10^{15} \mathrm{~K}$ were observed with associated maser fluxes of $T_{b} \Delta \Omega \leq 10^{10}$ (Nedoluha \& Watson 1991). High degrees of linear polarization up to $75 \%$ were observed. Analysis of the relation between the polarization fraction and the maser brightness for the highly polarized strongest feature shows a decline in polarization with the maser intensity. This is in line with an anisotropically pumped maser at high brightness beyond $\left(T_{b} \Delta \Omega\right)_{\text {mag. sat }}$.

Circular polarization up to $\sim 2 \%$ for these strong maser flares was also detected (Fiebig \& Güsten 1989). Fiebig \& Güsten (1989) also included the masers of a number of other starforming regions in their sample. Stokes $V$ spectra show the characteristic S-shaped spectra, which is an anomaly for water 


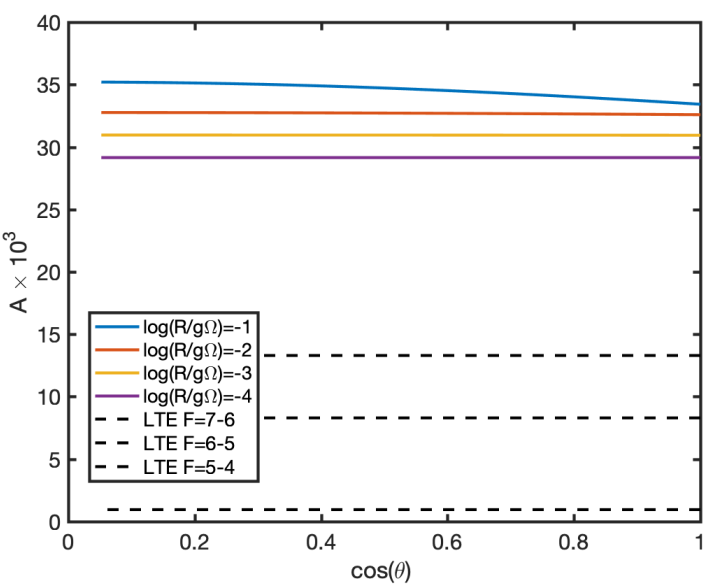

(a)

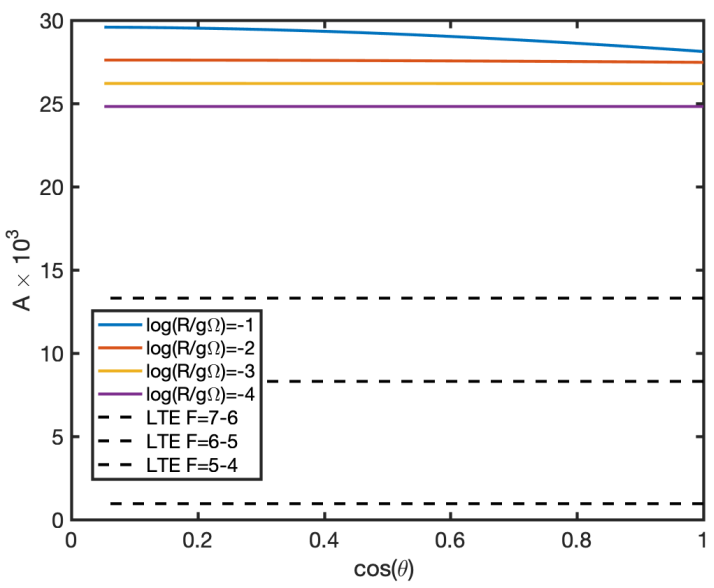

(b)

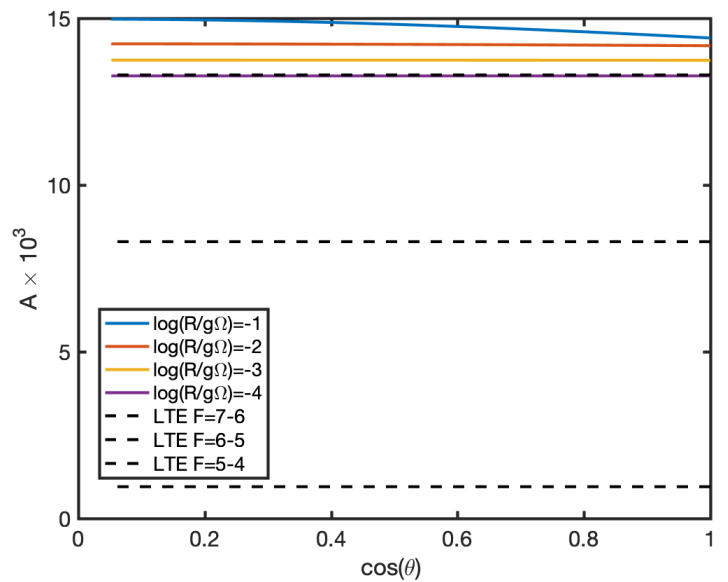

(c)

Fig. 15. The $A_{F F^{\prime}}$ coefficients of an isotropically pumped water maser at $B=20 \mathrm{G}$ as a function of the magnetic field-propagation direction angle $\cos \theta$. The panels show different thermal widths $v_{\text {th }}=$ (a) $0.6 \mathrm{~km} \mathrm{~s}^{-1}$, (b) $1.0 \mathrm{~km} \mathrm{~s}^{-1}$, and (c) $2.0 \mathrm{~km} \mathrm{~s}^{-1}$. Plots are given for different $\log (R / g \Omega)$. The LTE solutions (constant over $\cos \theta$ ) of the different hyperfine subtransitions are indicated by a dotted line.

masers that we found to occur only for preferably pumped water masers where one hyperfine transition dominates the others. The spread in circular polarization fractions can be explained by the variable projection of the magnetic field $(10-100 \mathrm{mG})$ onto the propagation axis, and variable magnetic fields in the sources. Vlemmings et al. $(2001,2002)$ investigated the circular polarization of masers occurring in the circumstellar envelopes of late-type stars. Magnetic fields around these masers are expected to be strong $(\sim G)$, and circular polarization should thus be detectable in the stronger maser features. Circular polarization up to $13 \%$ is found, but this concerns a single outlier. The weaker masers show circular polarization up to $6 \%$, which can be generated by a magnetic field of $\sim 400 \mathrm{mG}$. Generally, circular polarization seems to decline with increasing maser brightness, but this might be an effect of the detection limit.

A large sample of polarization observations of water masers comes from VLBI measurements around the high-mass starforming region $\mathrm{W} 75 \mathrm{~N}$ (Surcis et al. 2011). Here, for 17 maser features, significant linear and circular polarization is found. Linear polarization tends to be low $<10 \%$, but relatively high circular polarization $(<3 \%)$ is found. In part, the large fraction of highly circularly polarized masers is due to observational bias against weakly polarized masers. A similar scatter analysis to that performed for the $\mathrm{SiO}$ maser sample of Herpin et al. (2006), assuming that the water maser is pumped isotropically with no hyperfine-preference, at a thermal width of $\Delta v_{\mathrm{th}}=1 \mathrm{~km} \mathrm{~s}^{-1}$, and the magnetic field is randomly oriented per maser, shows that a magnetic field of $\sim 200 \mathrm{mG}$ best reproduces the obtained linearto-circular polarization distribution (see Fig. 14).

\section{Conclusions}

In this paper, we present CHAMP, a program that performs onedimensional numerical maser polarization simulations of nonparamagnetic molecules. Simulations are possible for masers with arbitrary high angular momentum transitions. Also, multiple close-lying hyperfine transitions that contribute to the same maser can be included in our model. Simulation of the polarization of complex and highly excited masers will become more relevant in the era of ALMA and its full polarization capabilities.

Illustrative calculations of the $\mathrm{SiO}$ and water masers reveal the following general observations about the polarization of masers:

- Linear polarization is mostly absent when the rate of stimulated emission is lower than the isotropic decay $\left(T_{b} \Delta \Omega<\right.$ $\left.\left(T_{b} \Delta \Omega\right)_{\text {sat }}\right)$. If polarization occurs for such weak masers, alternative polarizing mechanisms are in play. Circular polarization, however, is present for such weak masers and comes from the Zeeman effect. A LTE analysis of the Zeeman effect will give a reasonable estimate of the polarizing effects, but this approximation worsens with the maser brightness.

- The $90^{\circ}$ polarization angle flip at the magic angle $\theta_{\mathrm{m}}$, predicted by GKK73, is sharp only in the limit, $g \Omega \gg R$, when the magnetic precession rate is far greater than the rate of stimulated emission. However, for $g \Omega / R<100$, the $90^{\circ}$ flip is gradual and significant polarization is also found at propagation at the magic angle $\theta_{\mathrm{m}}$.

- Anisotropic pumping of a maser can lead to arbitrarily high linear polarization fractions, but will only be weakly associated with circular polarization. One characteristic of an anisotropically pumped weak maser is a linear growth in linear polarization fraction as a function of the maser brightness.

- Incident polarized seed radiation maintains its polarization degree until the rate of stimulated emission becomes comparable to the magnetic precession rate. From here, it slowly converges to the standard isotropic polarization solution.

- Circular polarization fractions are highest in the region where the rate of stimulated emission is on the same order 
as the magnetic precession rate. Circular polarization in this regime is associated with high linear polarization. Weak masers are weakly polarized, with a polarizing effect similar to thermal polarization.

- Overall polarization will drop strongly between the $J=1-0$ and $J=2-1$ transitions. The polarization of transitions with increasing angular momentum will gradually deteriorate.

A cursory overview of existing maser polarization observations leads to a reinforcement of the idea that highly polarized $\mathrm{SiO}$ masers are the product of anisotropic pumping. A similar mechanism probably underlies the highly polarized water super maser at Orion, which also showed a drop in polarization with maser brightness, as predicted by our theories. We show that comparing theoretical $p_{\mathrm{L}}-p_{\mathrm{V}}$ scatter plots to the observationally obtained $p_{\mathrm{L}}-p_{\mathrm{V}}$ scatter, can be a promising method to ascertain the overall magnetic field strength of a region with a large number of masers. Finally, we find that the variation in the polarization angle across a maser spectrum can be used as a proxy for the rate of stimulated emission. This would be an important additional measure to determine the maser saturation level and beaming angle, which are difficult to observe directly.

Acknowledgements. Support for this work was provided by the the Swedish Research Council (VR), and by the European Research Council under the European Union's Seventh Framework Programme (FP7/2007-2013), through the ERC consolidator grant agreement nr. 614264. Simulations were performed on resources at the Chalmers Centre for Computational Science and Engineering (C3SE) provided by the Swedish National Infrastructure for Computing (SNIC).

\section{References}

Amiri, N., Vlemmings, W., Kemball, A., \& Van Langevelde, H. 2012, A\&A, 538, A136

Anderson, E., Bai, Z., Bischof, C., et al. 1999, LAPACK Users' Guide, 3rd edn. (Philadelphia, PA: Society for Industrial and Applied Mathematics)

Assaf, K., Diamond, P., Richards, A., \& Gray, M. 2013, MNRAS, 431, 1077

Barvainis, R., McIntosh, G., \& Predmore, C. R. 1987, Nature, 329, 613

Baudry, A., \& Diamond, P. 1998, A\&A, 331, 697

Cotton, W., Mennesson, B., Diamond, P., et al. 2004, A\&A, 414, 275

Cotton, W., Ragland, S., \& Danchi, W. 2011, ApJ, 736, 96

Degl'Innocenti, M. L., \& Landolfi, M. 2006, Polarization in Spectral Lines (Springer Science \& Business Media), 307

Deguchi, S., \& Watson, W. D. 1990, ApJ, 354, 649

Elitzur, M. 1991, ApJ, 370, 407

Elitzur, M. 1992, ARA\&A, 30, 75
Elitzur, M. 1993, ApJ, 416, 256

Elitzur, M. 1995, ArXiv e-prints [arxiv:astro-ph/9508007]

Elitzur, M. 1998, ApJ, 504, 390

Elitzur, M., Hollenbach, D. J., \& McKee, C. F. 1992, ApJ, 394, 221

Fiebig, D., \& Güsten, R. 1989, A\&A, 214, 333

Fish, V. L., \& Reid, M. J. 2006, Ap\&SS., 164, 99

Garay, G., Moran, J., \& Haschick, A. 1989, ApJ, 338, 244

Goldreich, P., Keeley, D. A., \& Kwan, J. Y. 1973, ApJ, 179, 111

Gray, M. 2003, MNRAS, 343, L33

Gray, M. 2012, Maser Sources in Astrophysics (Cambridge University Press), 50 Gray, M., \& Field, D. 1995, A\&A, 298, 243

Herpin, F., Baudry, A., Thum, C., Morris, D., \& Wiesemeyer, H. 2006, A\&A, 450,667

Houde, M. 2014, ApJ, 795, 27

Houde, M., Hezareh, T., Jones, S., \& Rajabi, F. 2013, ApJ, 764, 24

Kemball, A., \& Diamond, P. 1997, ApJ, 481, L111

Kemball, A. J., Diamond, P. J., Gonidakis, I., et al. 2009, ApJ, 698, 1721

Lankhaar, B., Groenenboom, G. C., \& van der Avoird, A. 2016, J. Chem. Phys., 145,244301

Lankhaar, B., Vlemmings, W., Surcis, G., et al. 2018, Nat. Astron., 2, 145

Nedoluha, G. E., \& Watson, W. D. 1990, ApJ, 354, 660

Nedoluha, G. E., \& Watson, W. D. 1991, ApJ, 367, L63

Nedoluha, G. E., \& Watson, W. D. 1992, ApJ, 384, 185

Nedoluha, G. E., \& Watson, W. D. 1994, ApJ, 423, 394

Pérez-Sánchez, A., \& Vlemmings, W. 2013, A\&A, 551, A15

Plambeck, R., Wright, M., \& Rao, R. 2003, ApJ, 594, 911

Richards, A., Elitzur, M., \& Yates, J. 2011, A\&A, 525, A56

Richter, L., Kemball, A., \& Jonas, J. 2016, MNRAS, 461, 2309

Robishaw, T., Quataert, E., \& Heiles, C. 2008, ApJ, 680, 981

Sobolev, A., Moran, J., Gray, M., et al. 2018, ApJ, 856, 60

Surcis, G., Vlemmings, W., Curiel, S., et al. 2011, A\&A, 527, A48

Tobin, T., Kemball, A., \& Gray, M. 2019, ApJ, 871, 189

Trung, D.-V. 2009, MNRAS, 399, 1495

Vlemmings, W. 2008, A\&A, 484, 773

Vlemmings, W., Diamond, P., \& Van Langevelde, H. 2001, A\&A, 375, L1

Vlemmings, W., Diamond, P., \& Van Langevelde, H. 2002, A\&A, 394, 589

Vlemmings, W., Surcis, G., Torstensson, K., \& Van Langevelde, H. 2010 MNRAS, 404, 134

Vlemmings, W., Torres, R., \& Dodson, R. 2011a, A\&A, 529, A95

Vlemmings, W., Humphreys, E., \& Franco-Hernández, R. 2011b, ApJ, 728, 149

Vlemmings, W., Khouri, T., Martí-Vidal, I., et al. 2017, A\&A, 603, A92

Vlemmings, W. H., \& van Langevelde, H. J. 2005, A\&A, 434, 1021

Vlemmings, W. H. T., Diamond, P. J., van Langevelde, H. J., \& Torrelles, J. M. 2006a, A\&A, 448, 597

Vlemmings, W. H., Diamond, P. J., \& Imai, H. 2006b, Nature, 440, 58

Walker, R. 1984, ApJ, 280, 618

Watson, W., \& Wyld, H. 2001, ApJ, 558, L55

Western, L., \& Watson, W. 1983, ApJ, 275, 195

Western, L., \& Watson, W. 1984, ApJ, 285, 158

Wiebe, D., \& Watson, W. 1998, ApJ, 503, L71 


\section{Appendix A: Additional figures}

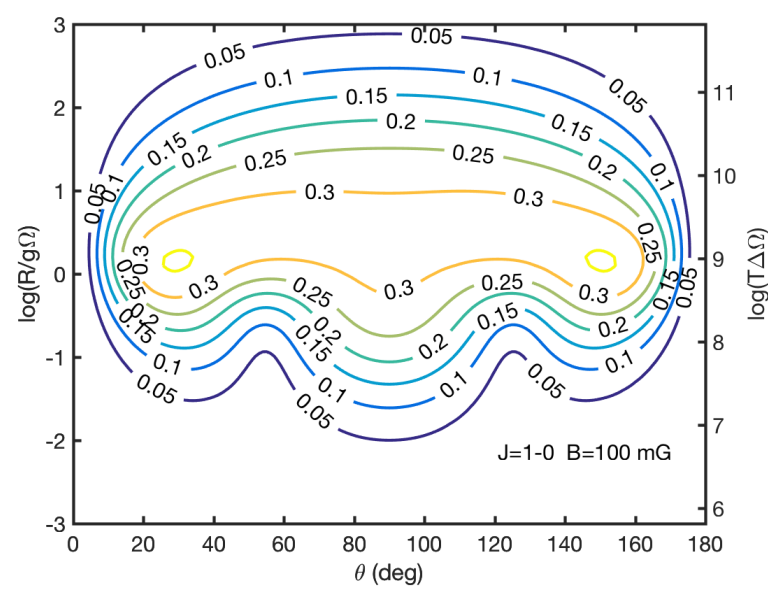

(a)

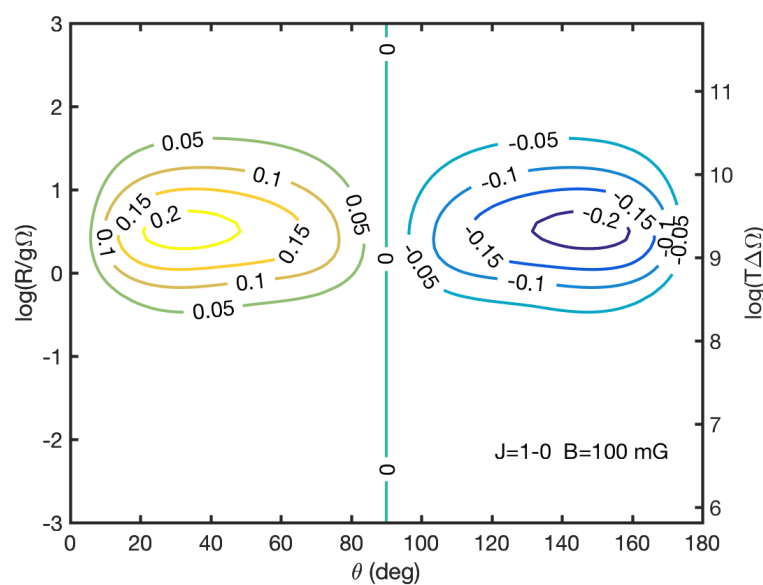

(c)

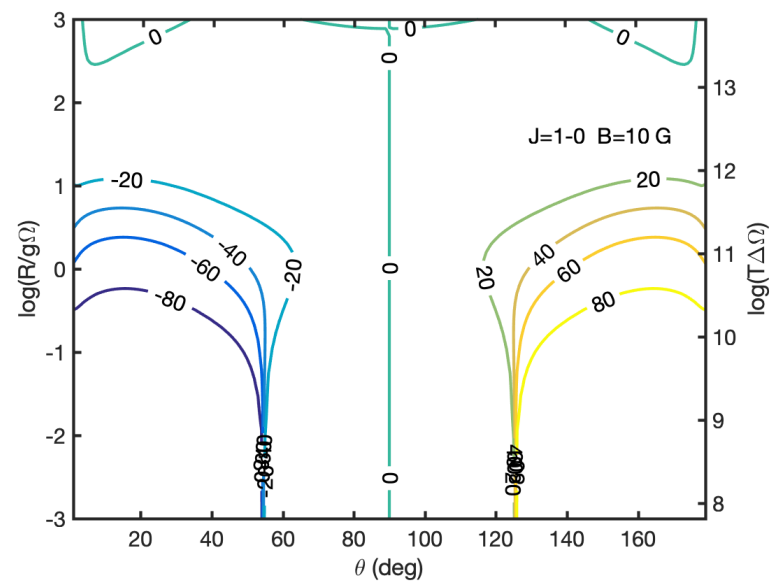

(e)

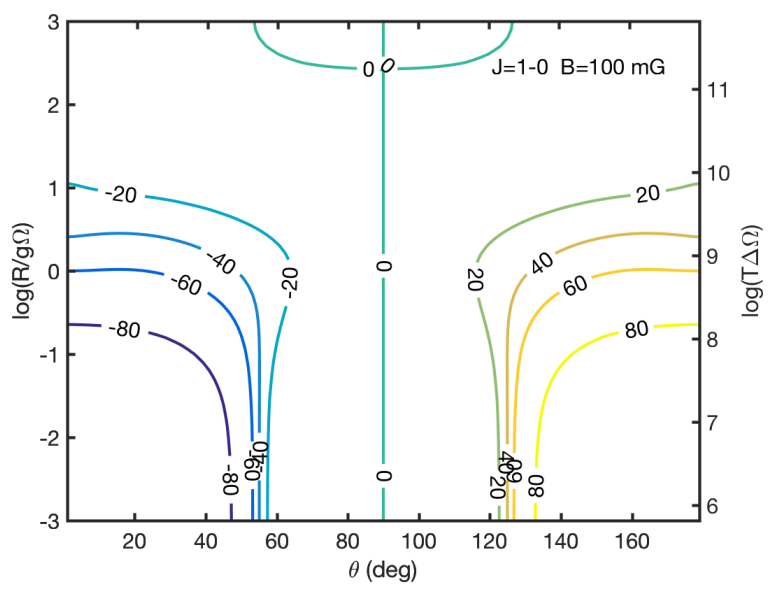

(b)

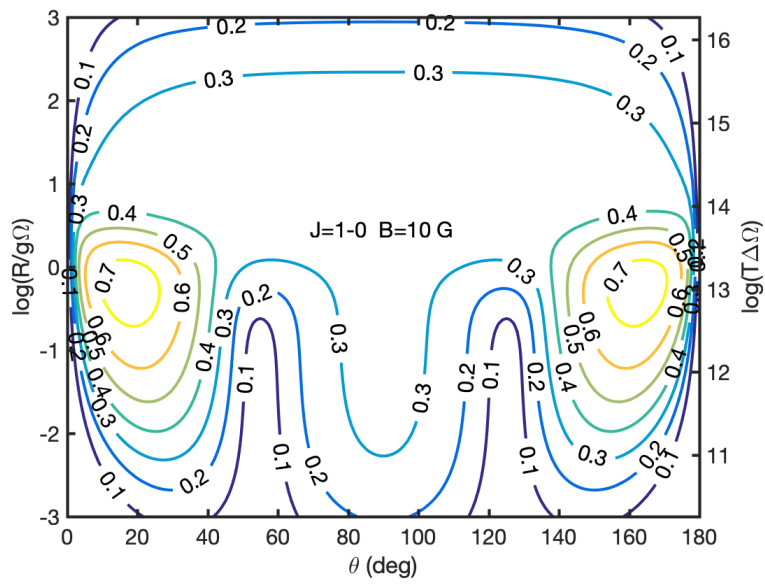

(d)

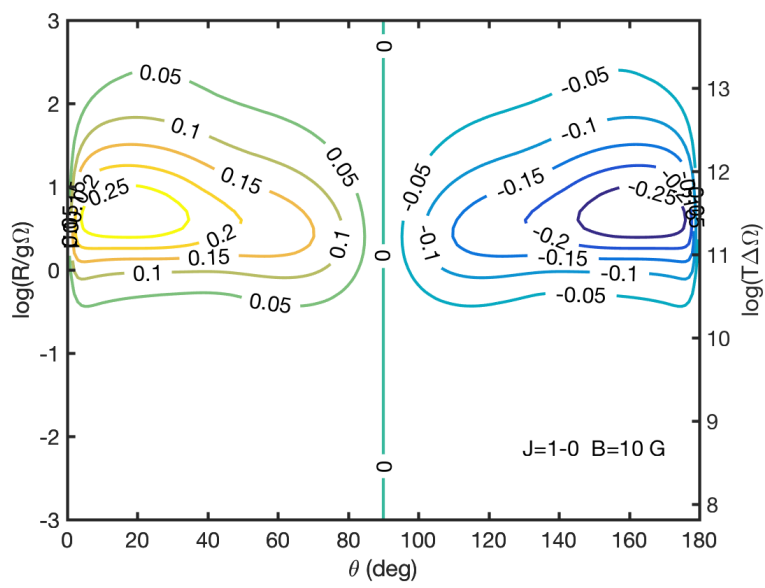

(f)

Fig. A.1. Simulations of an isotropically pumped $\mathrm{SiO}$ maser. Linear polarization fraction (a,d) and angle (b,e), and circular polarization fraction $(\mathrm{c}, \mathrm{f})$. Magnetic field strength and transition angular momentum are indicated. 
B. Lankhaar and W. Vlemmings: Maser polarization

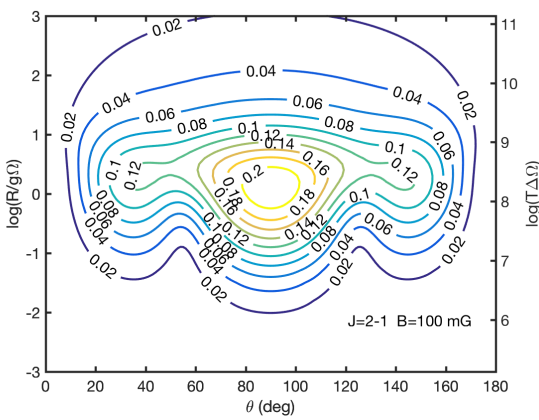

(a)

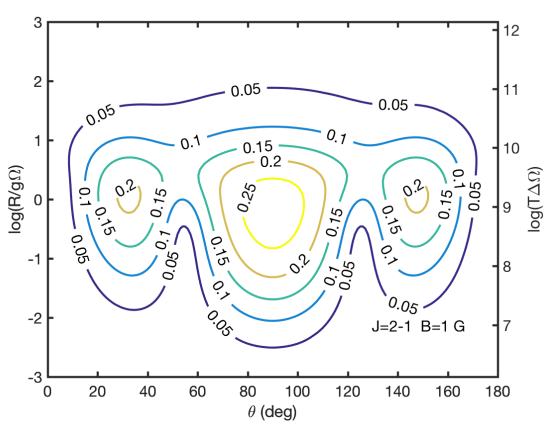

(d)

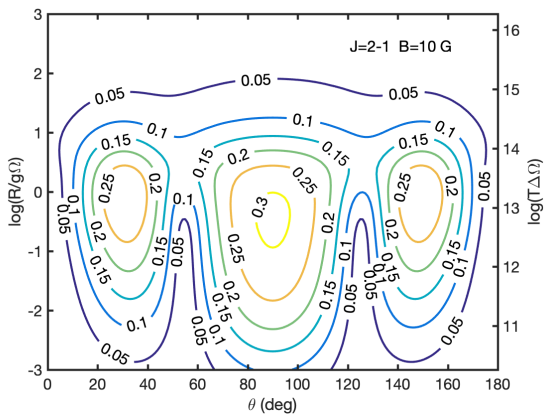

(g)

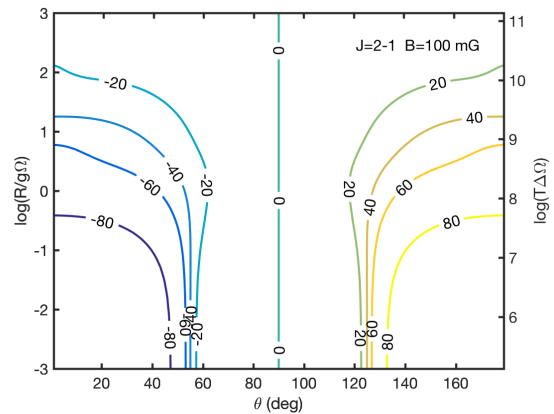

(b)

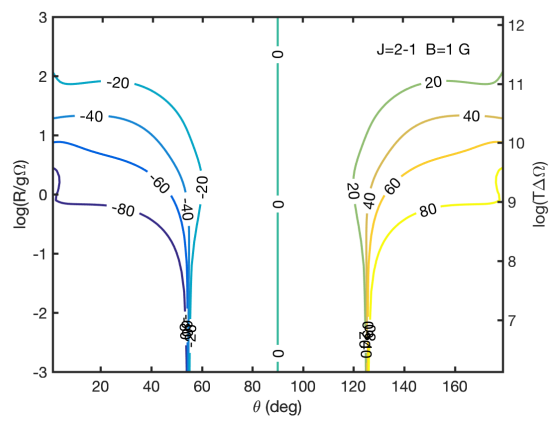

(e)

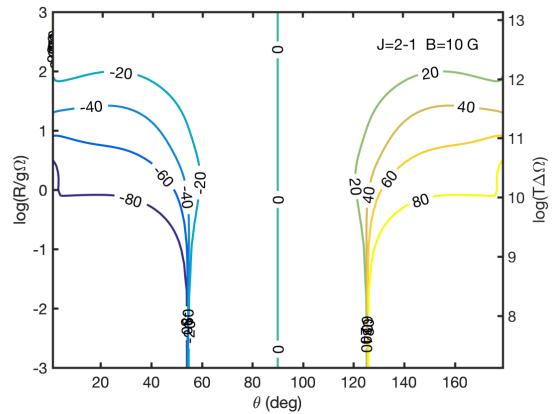

(h)

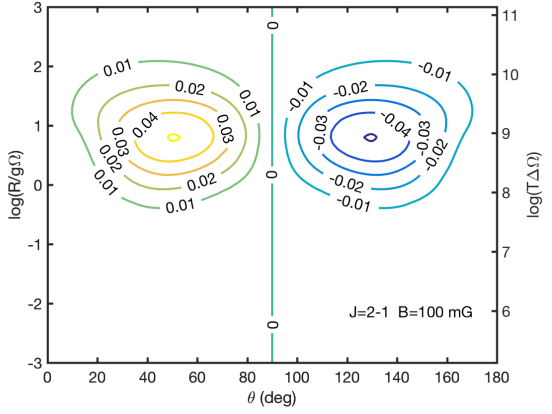

(c)

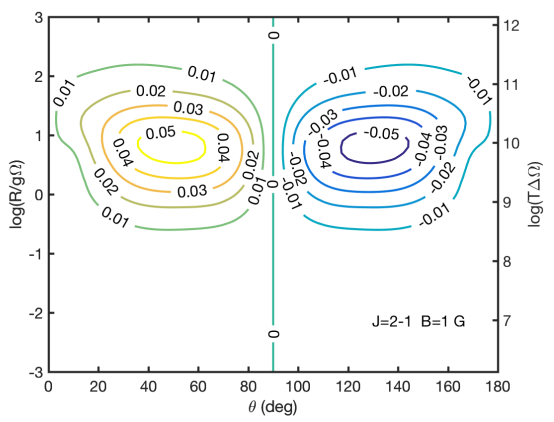

(f)

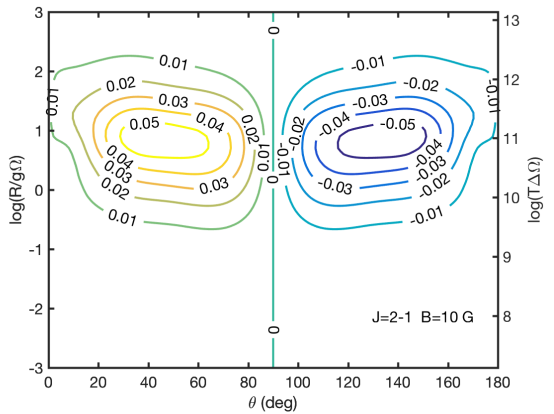

(i)

Fig. A.2. Simulations of an isotropically pumped $\mathrm{SiO}$ maser. Linear polarization fraction (a,d,g) and angle (b,e,h), and circular polarization fraction $(\mathrm{c}, \mathrm{f}, \mathrm{i})$. Magnetic field strength and transition angular momentum are indicated. 


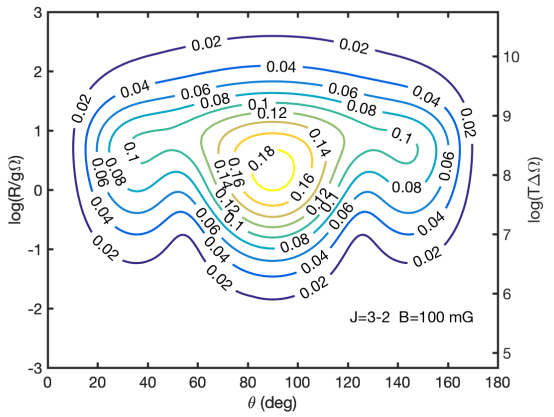

(a)

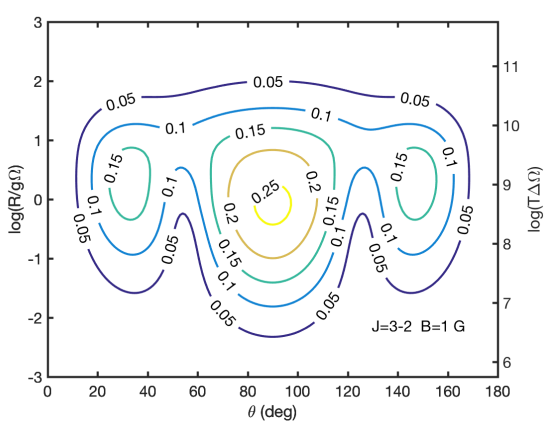

(d)

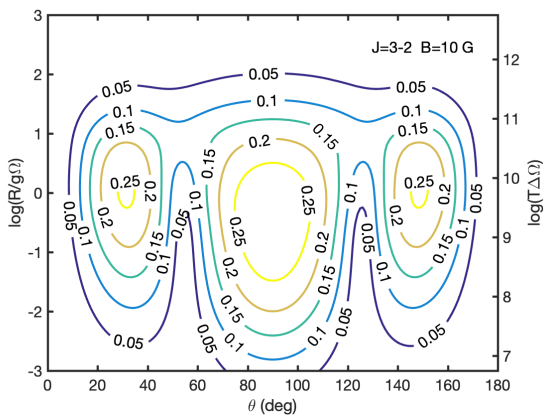

(g)

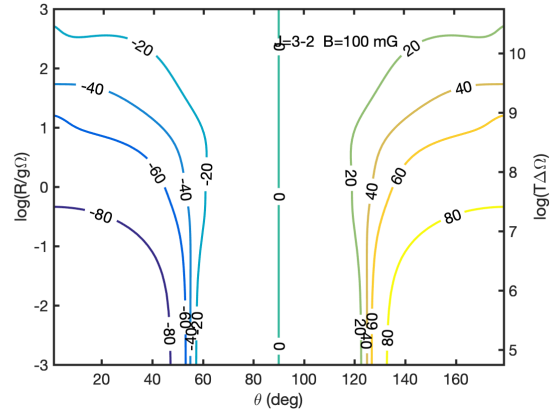

(b)

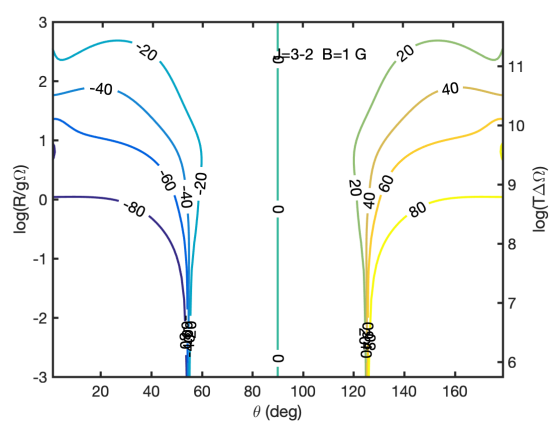

(e)

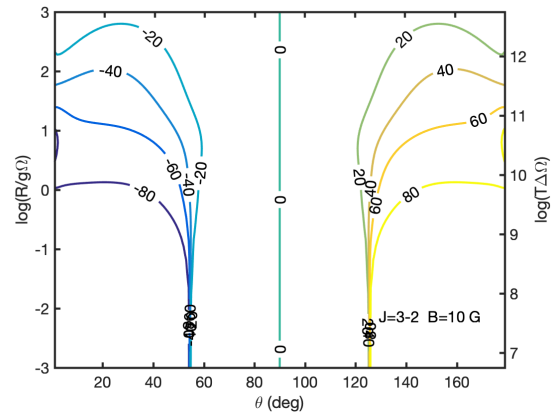

(h)

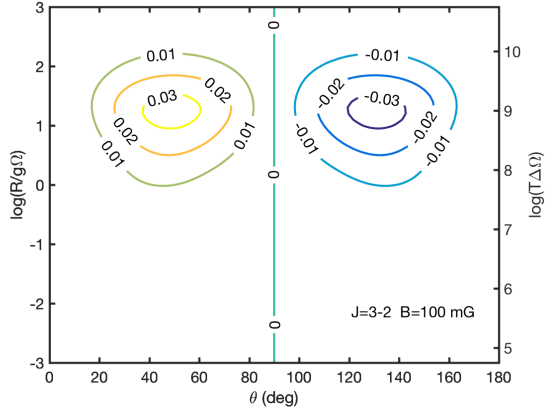

(c)

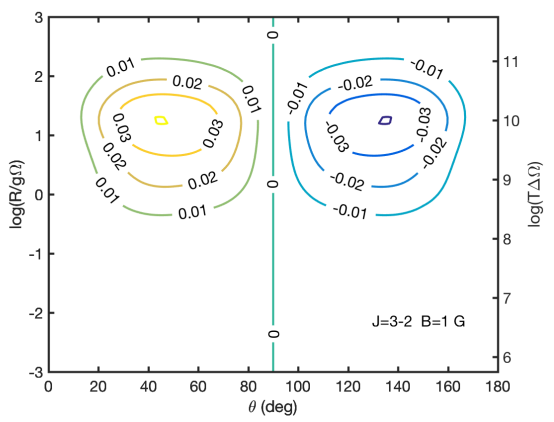

(f)

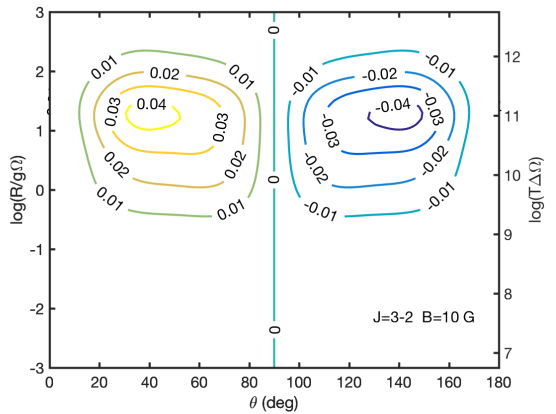

(i)

Fig. A.3. Simulations of an isotropically pumped $\mathrm{SiO}$ maser. Linear polarization fraction (a,d,g) and angle (b,e,h), and circular polarization fraction $(\mathrm{c}, \mathrm{f}, \mathrm{i})$. Magnetic field strength and transition angular momentum are indicated. 


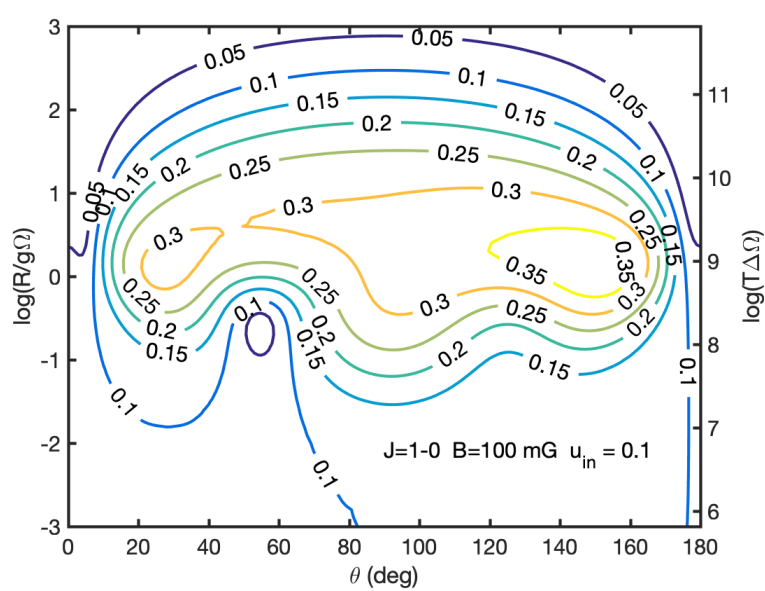

(a)

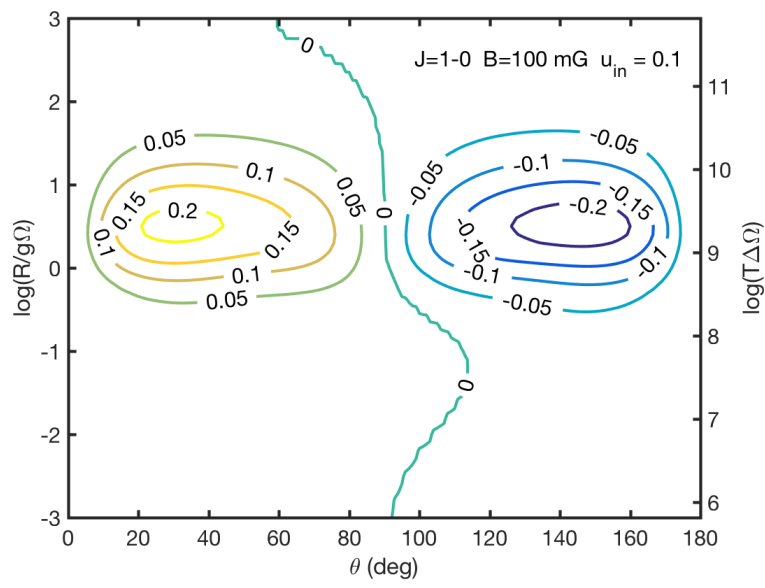

(c)

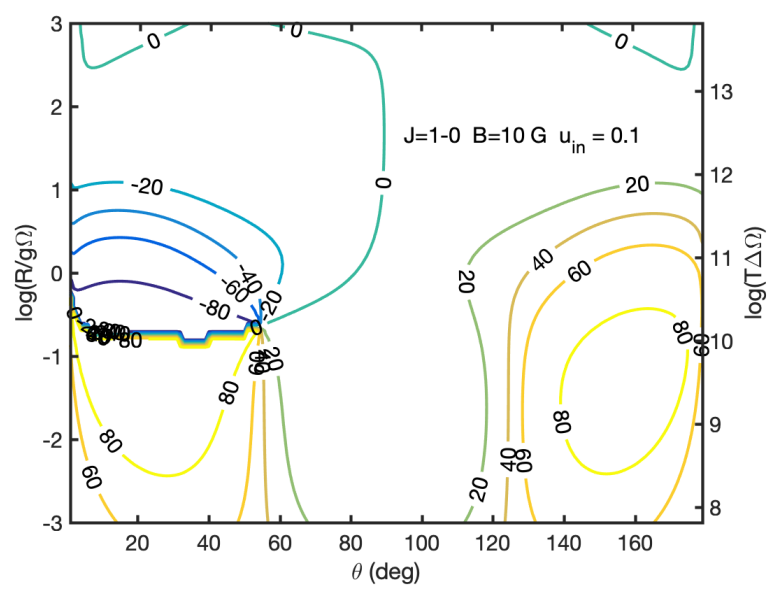

(e)

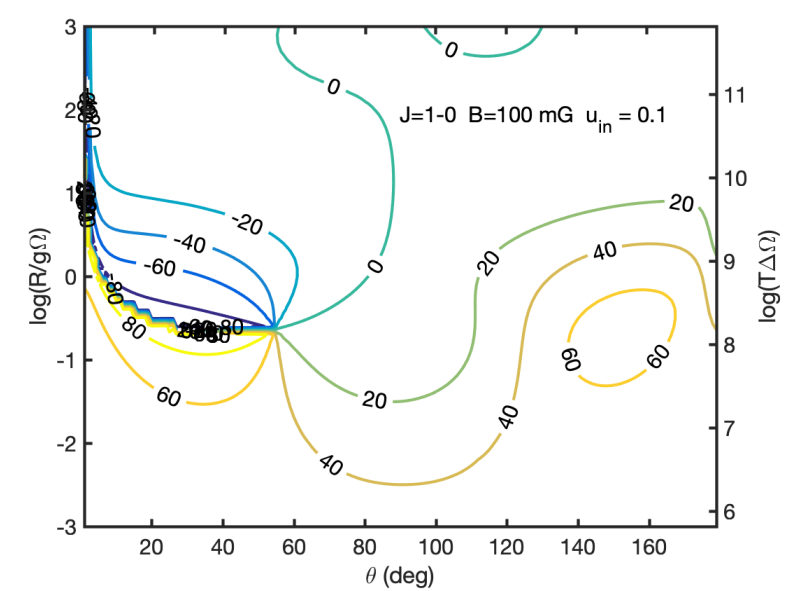

(b)

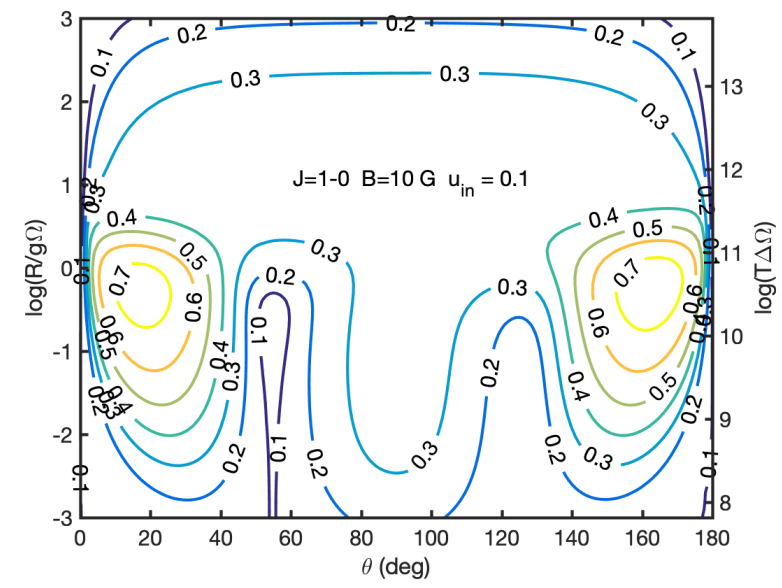

(d)

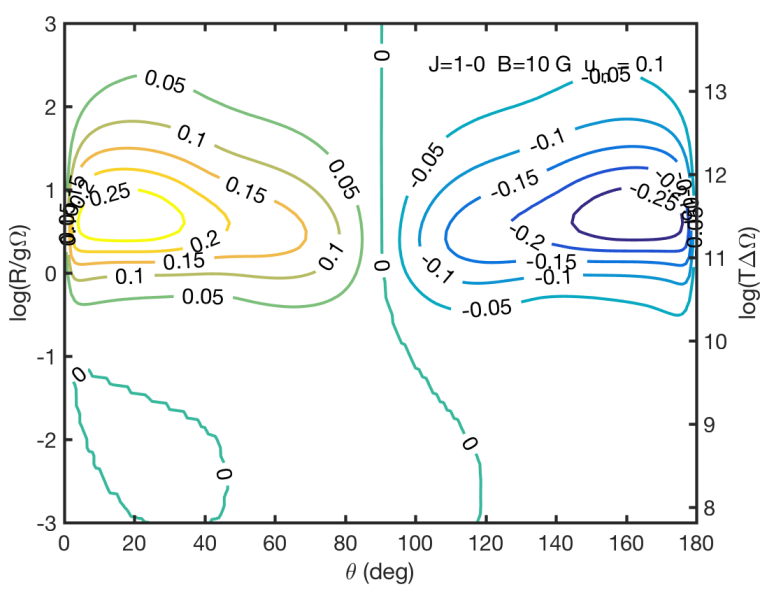

(f)

Fig. A.4. Simulations of a $\mathrm{SiO}$ maser with $10 \%$ polarized seed radiation. Linear polarization fraction $(\mathrm{a}, \mathrm{d})$ and angle (b,e), and circular polarization fraction $(\mathrm{c}, \mathrm{f})$. Magnetic field strength and transition angular momentum are indicated. 


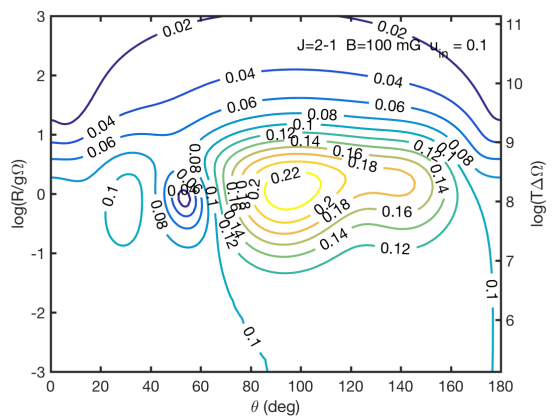

(a)

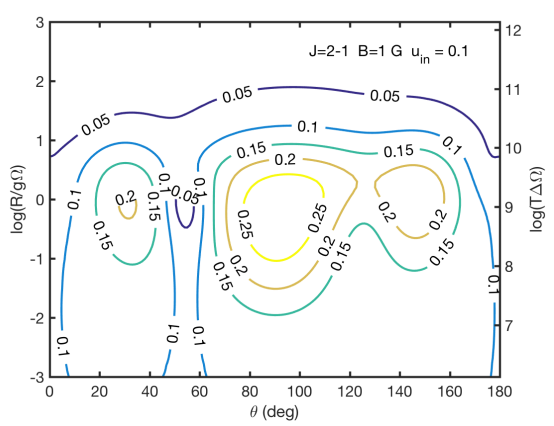

(d)

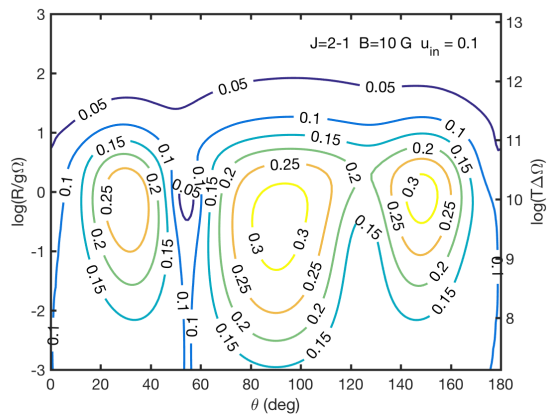

(g)

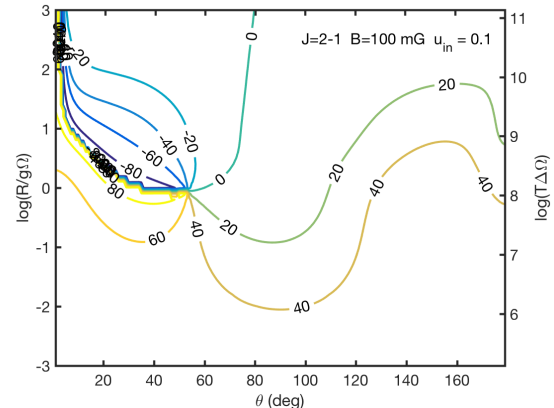

(b)

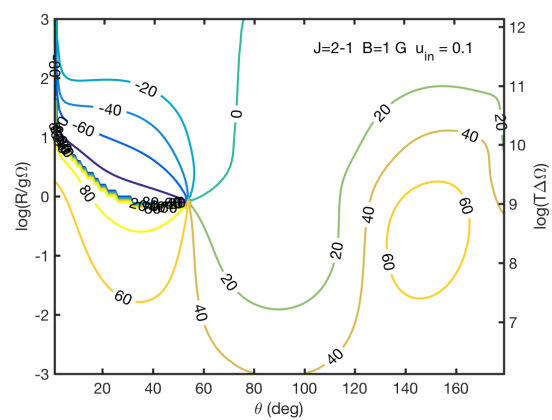

(e)

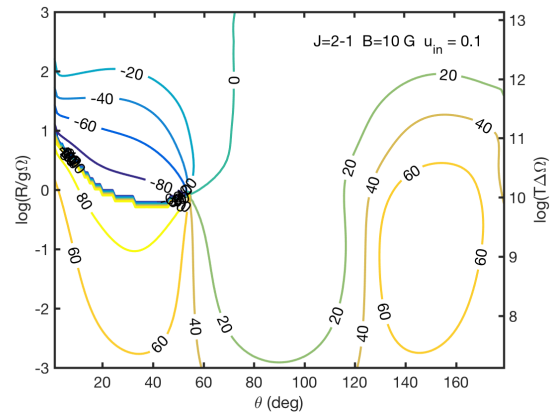

(h)

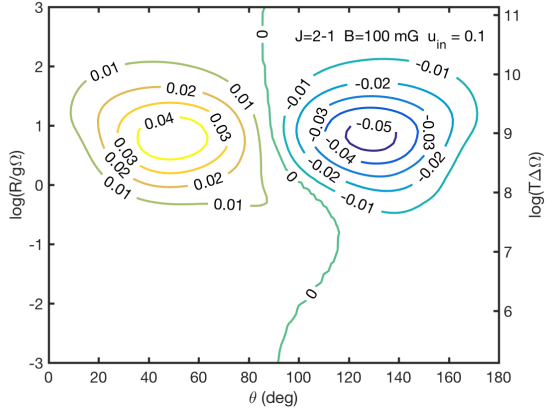

(c)

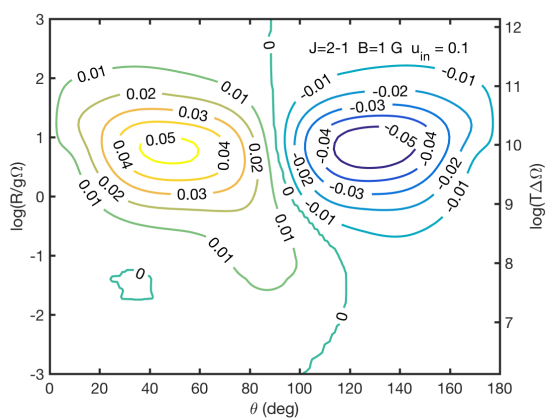

(f)

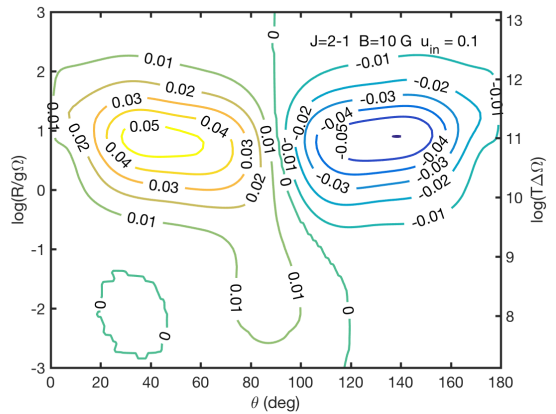

(i)

Fig. A.5. Simulations of a $\mathrm{SiO}$ maser with $10 \%$ polarized seed radiation. Linear polarization fraction (a,d,g) and angle (b,e,h), and circular polarization fraction $(\mathrm{c}, \mathrm{f}, \mathrm{i})$. Magnetic field strength and transition angular momentum are indicated. 
B. Lankhaar and W. Vlemmings: Maser polarization

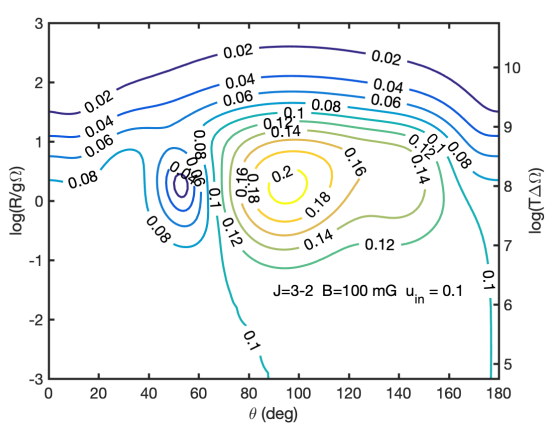

(a)

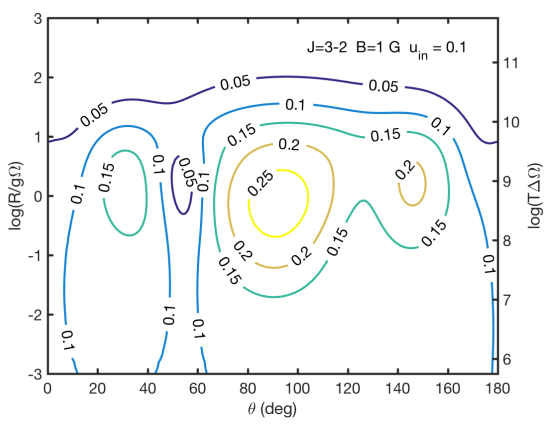

(d)

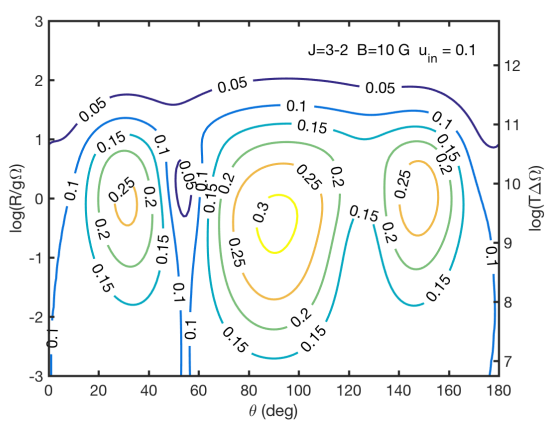

(g)

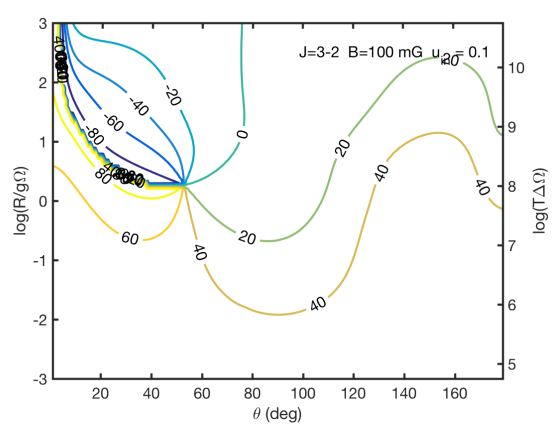

(b)

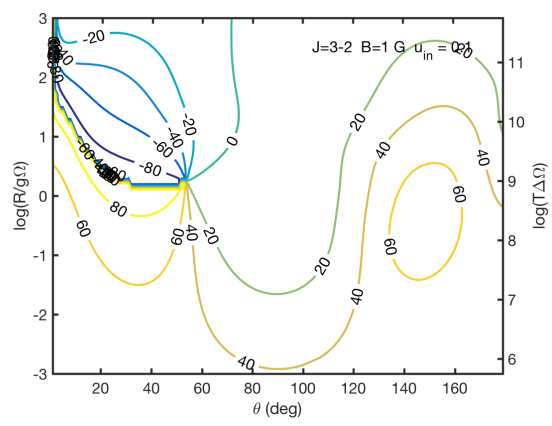

(e)

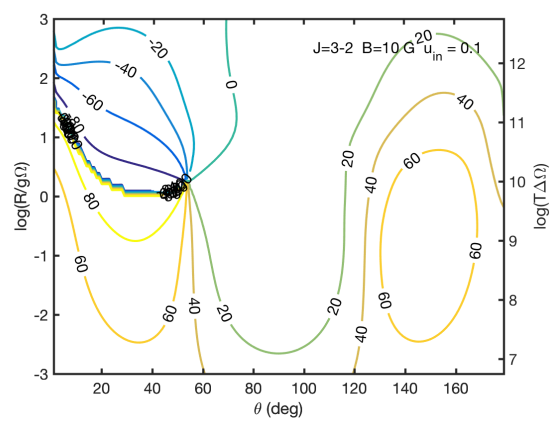

(h)

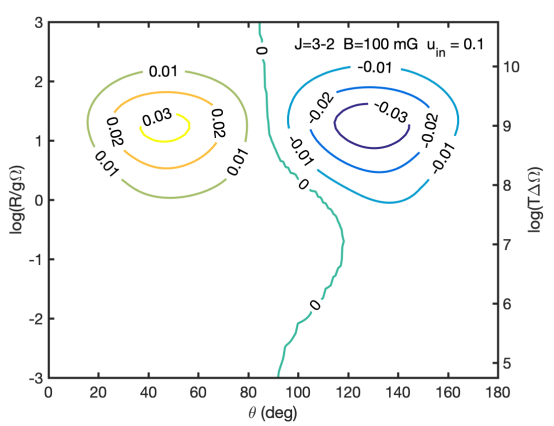

(c)

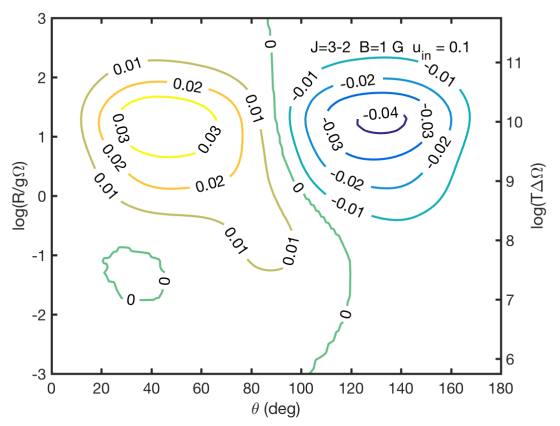

(f)

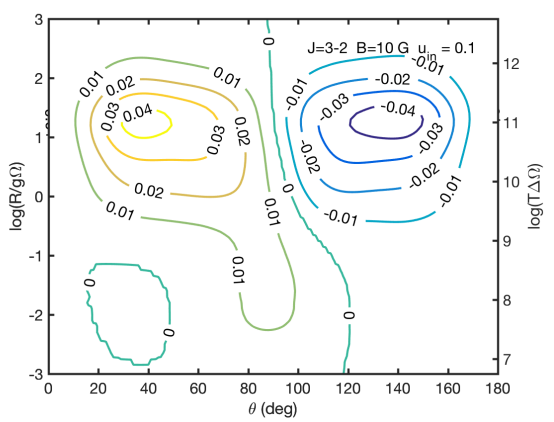

(i)

Fig. A.6. Simulations of a $\mathrm{SiO}$ maser with $10 \%$ polarized seed radiation. Linear polarization fraction (a,d,g) and angle (b,e,h), and circular polarization fraction $(\mathrm{c}, \mathrm{f}, \mathrm{i})$. Magnetic field strength and transition angular momentum are indicated. 


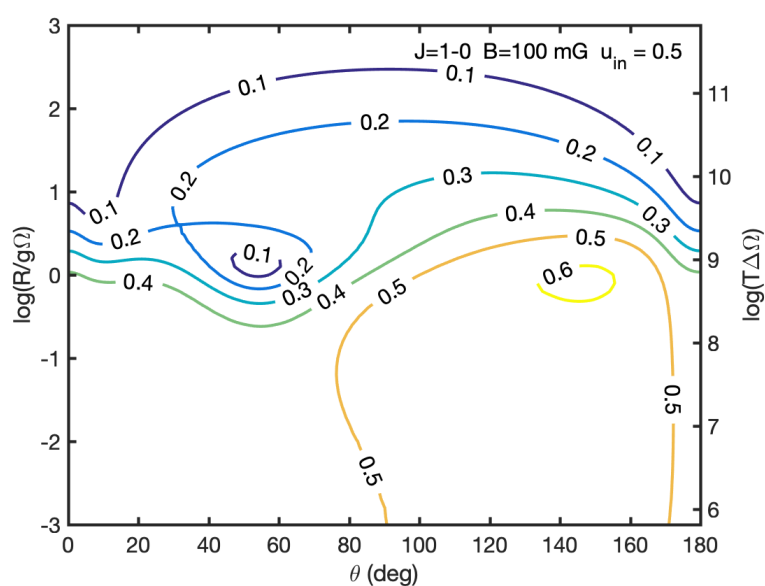

(a)

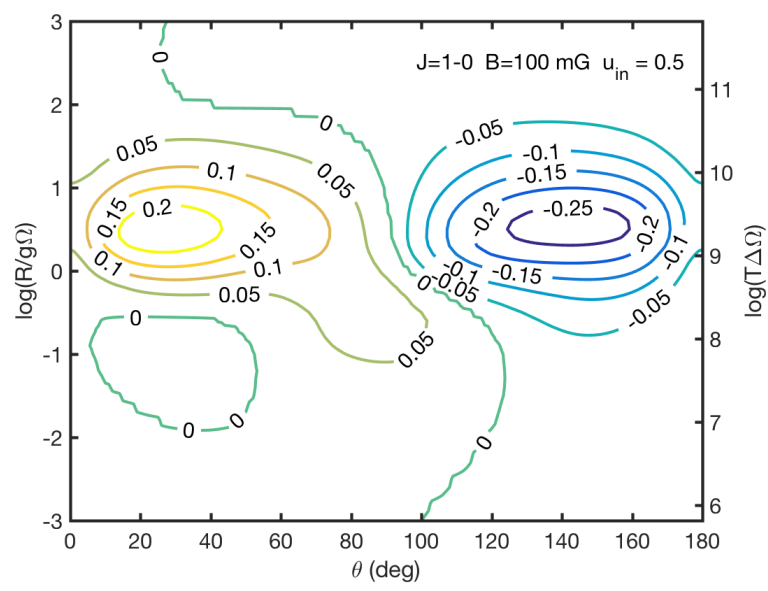

(c)

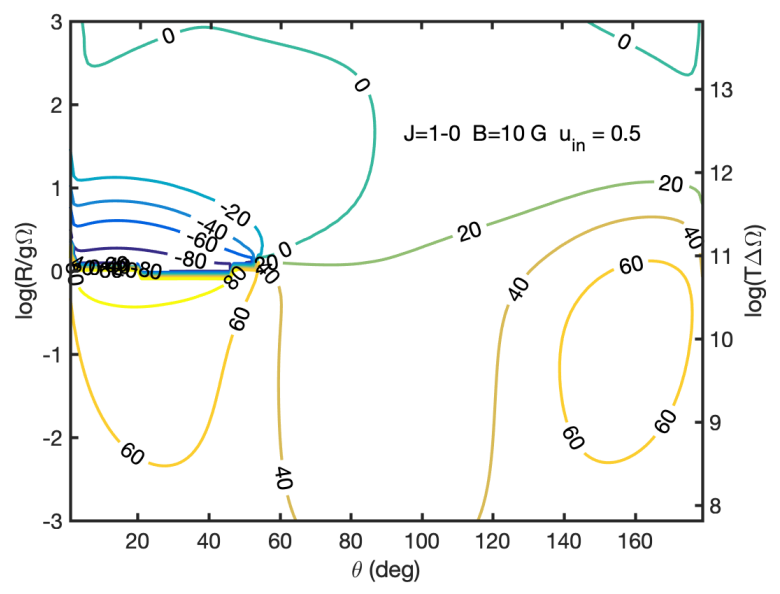

(e)

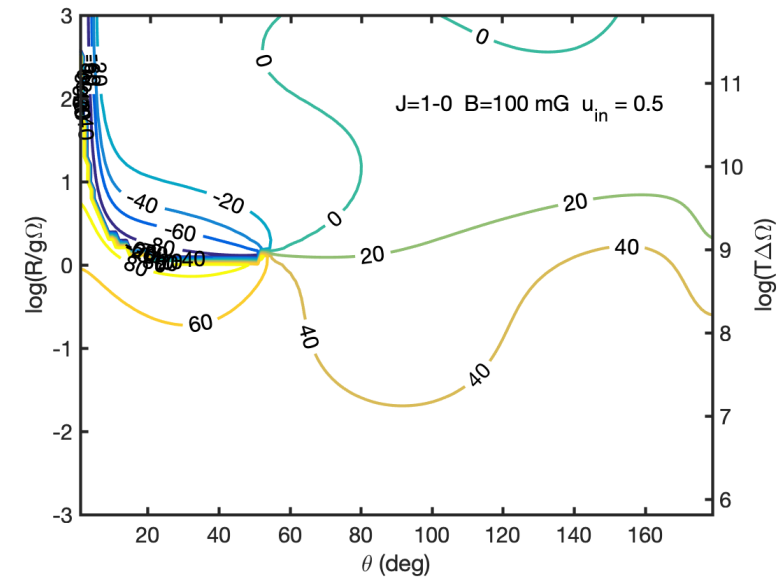

(b)

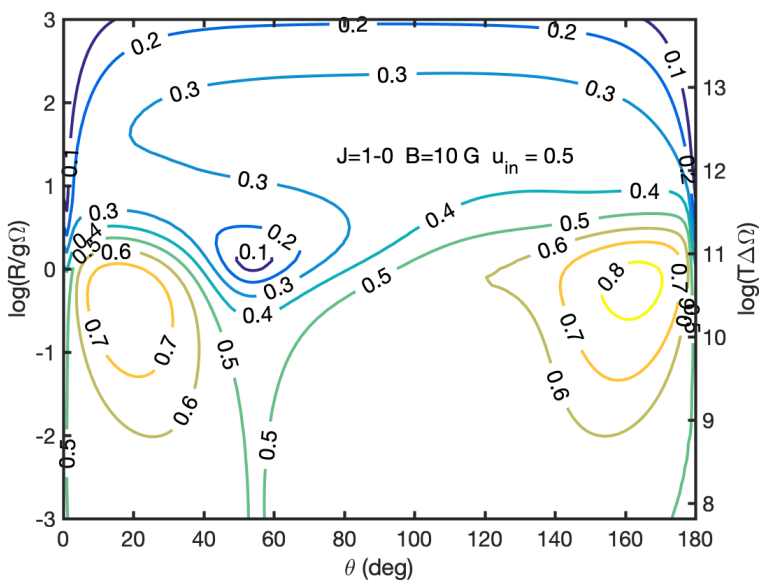

(d)

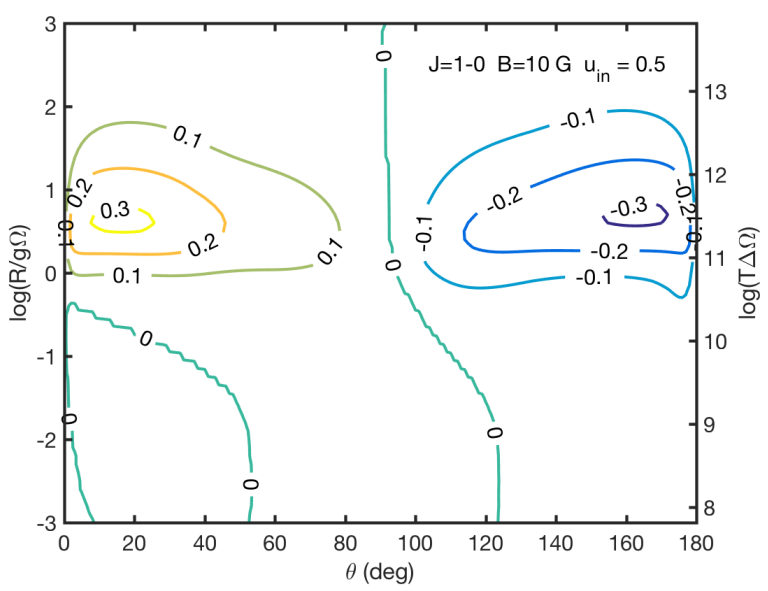

(f)

Fig. A.7. Simulations of a $\mathrm{SiO}$ maser with $50 \%$ polarized seed radiation. Linear polarization fraction (a,d) and angle (b,e), and circular polarization fraction $(\mathrm{c}, \mathrm{f})$. Magnetic field strength and transition angular momentum are indicated. 
B. Lankhaar and W. Vlemmings: Maser polarization

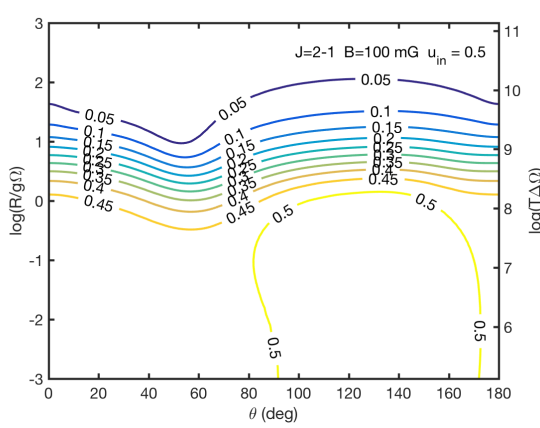

(a)

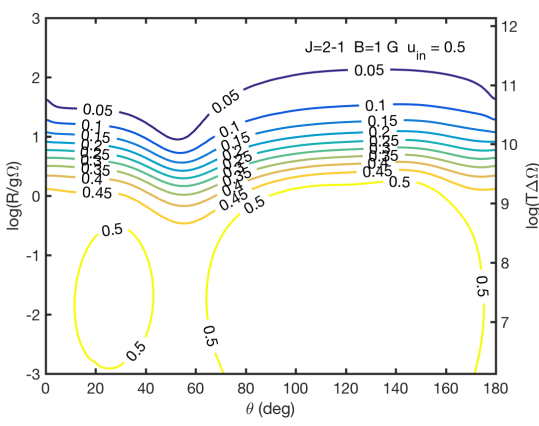

(d)

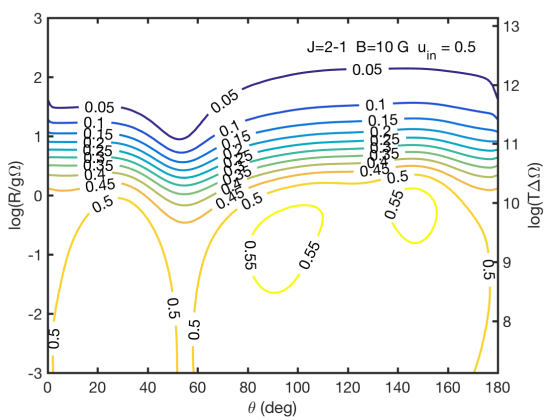

(g)

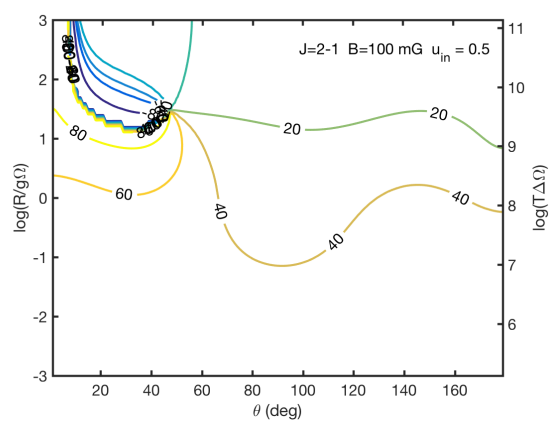

(b)

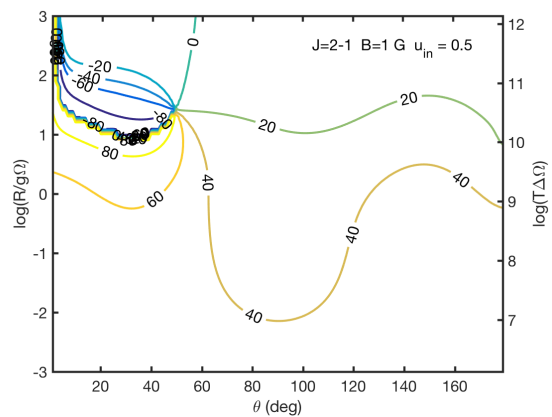

(e)

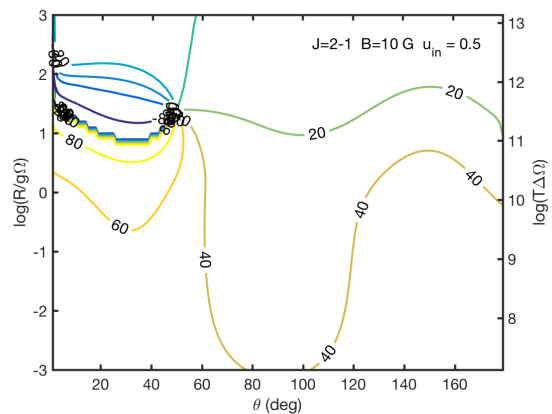

(h)

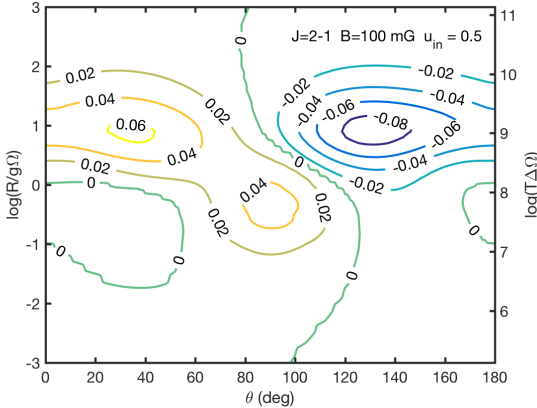

(c)

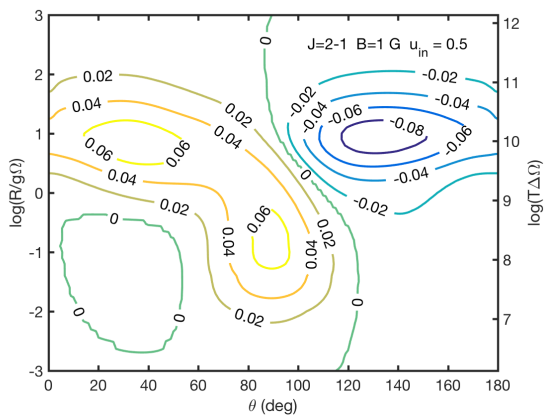

(f)

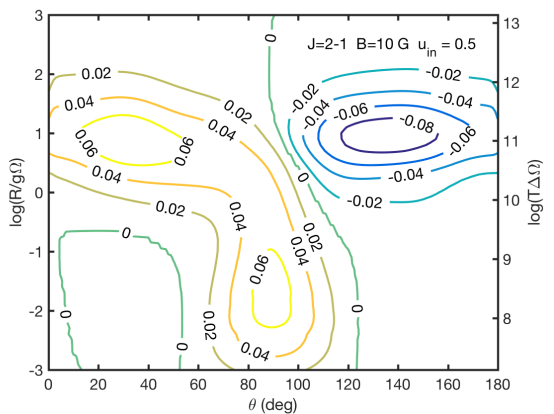

(i)

Fig. A.8. Simulations of a $\mathrm{SiO}$ maser with $50 \%$ polarized seed radiation. Linear polarization fraction (a,d,g) and angle (b,e,h), and circular polarization fraction $(\mathrm{c}, \mathrm{f}, \mathrm{i})$. Magnetic field strength and transition angular momentum are indicated. 


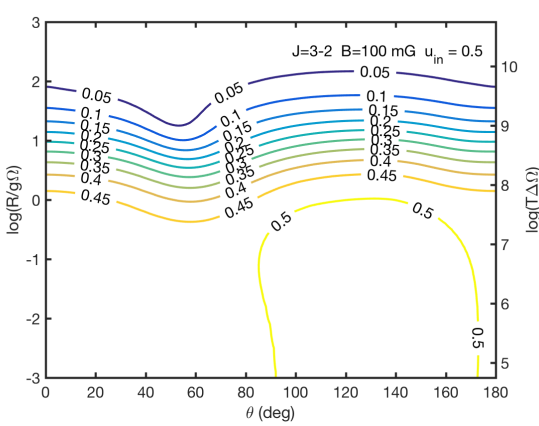

(a)

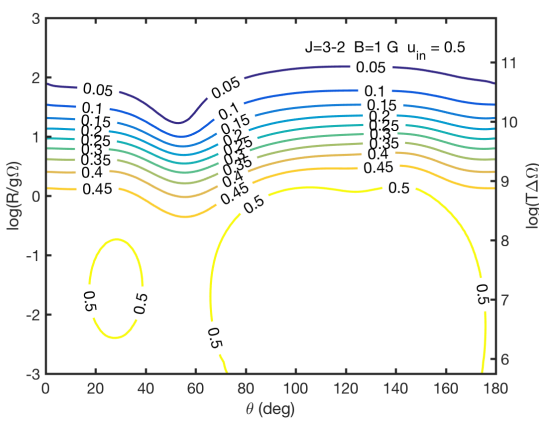

(d)

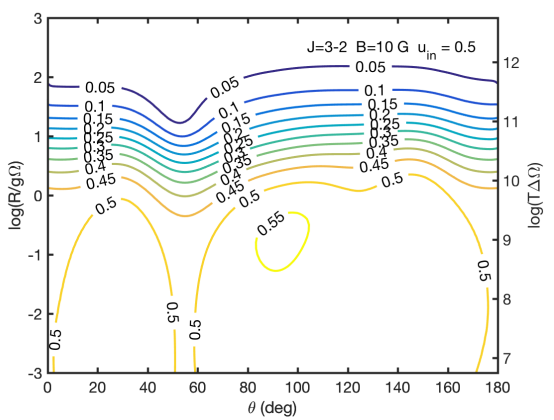

(g)

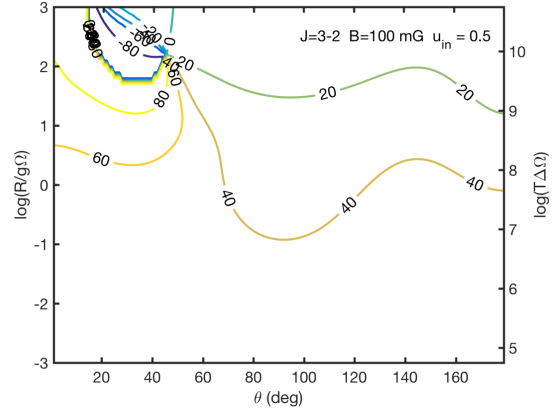

(b)

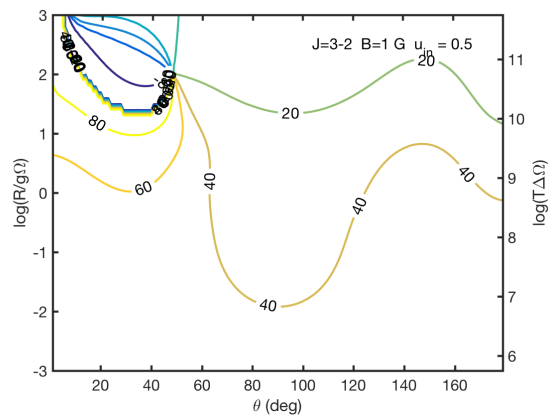

(e)

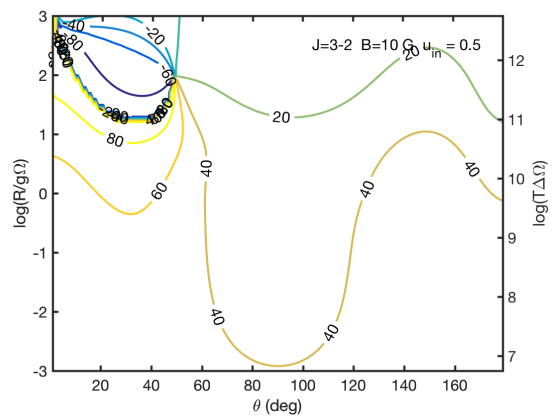

(h)

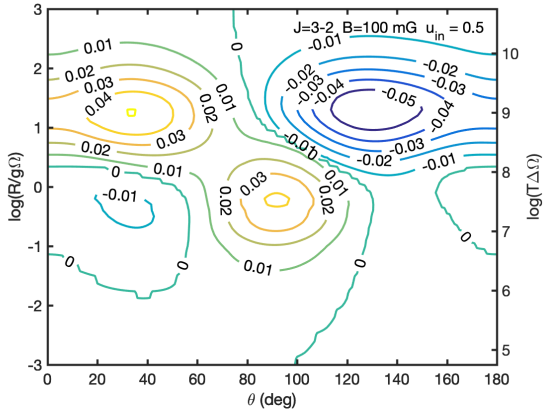

(c)

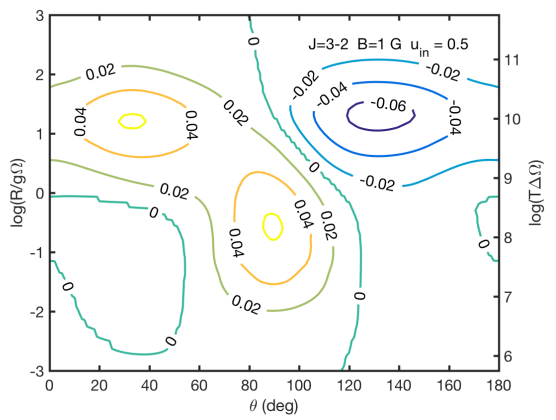

(f)

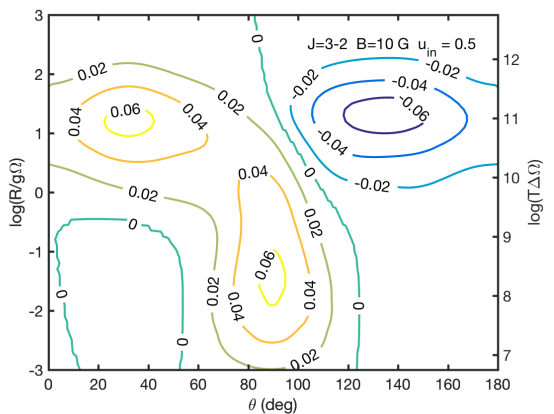

(i)

Fig. A.9. Simulations of a $\mathrm{SiO}$ maser with $50 \%$ polarized seed radiation. Linear polarization fraction (a,d,g) and angle (b,e,h), and circular polarization fraction $(\mathrm{c}, \mathrm{f}, \mathrm{i})$. Magnetic field strength and transition angular momentum are indicated. 


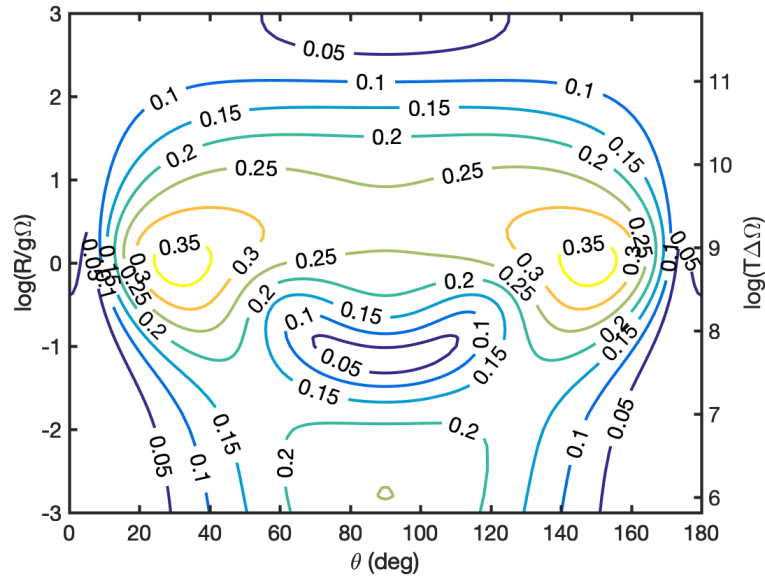

(a)

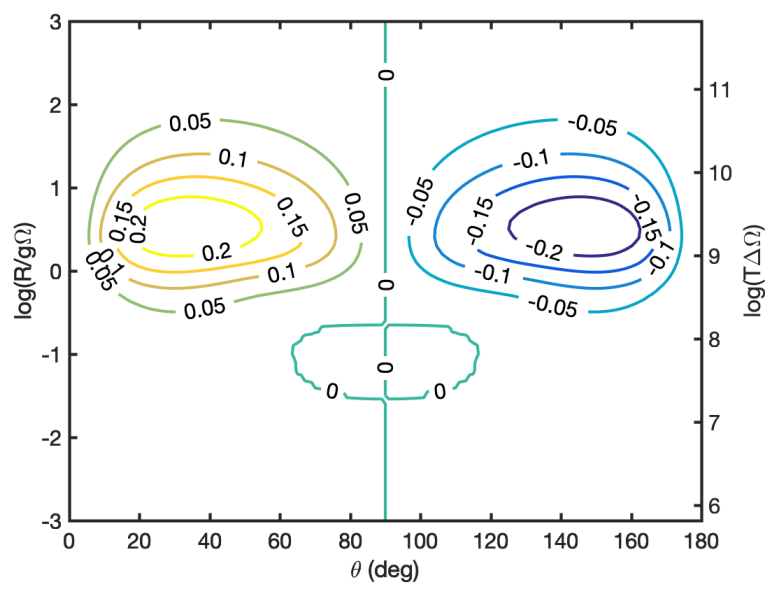

(c)

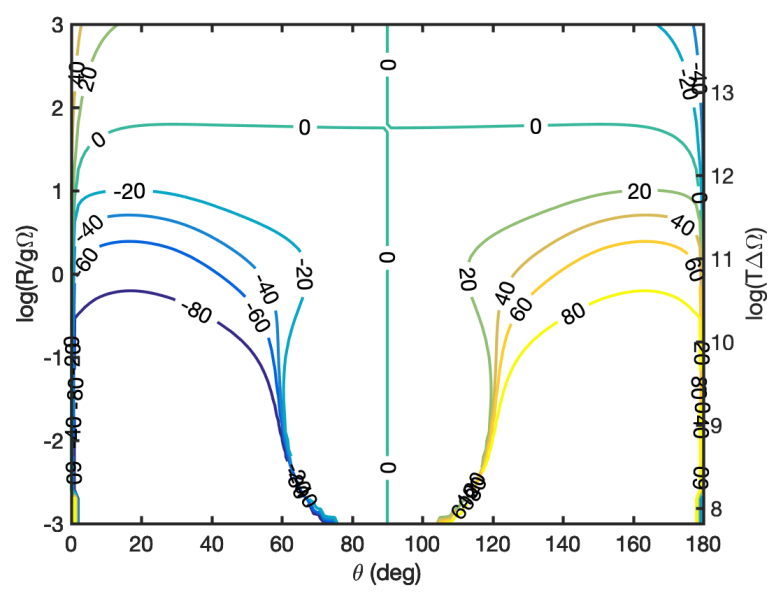

(e)

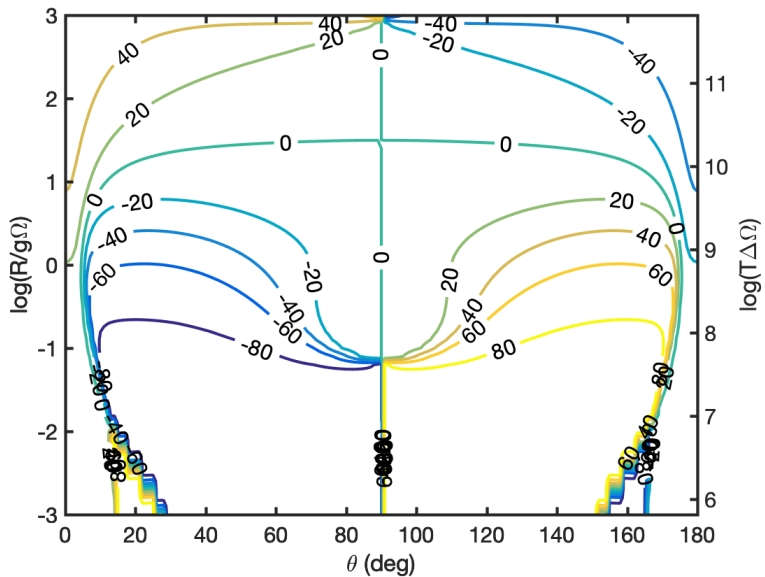

(b)

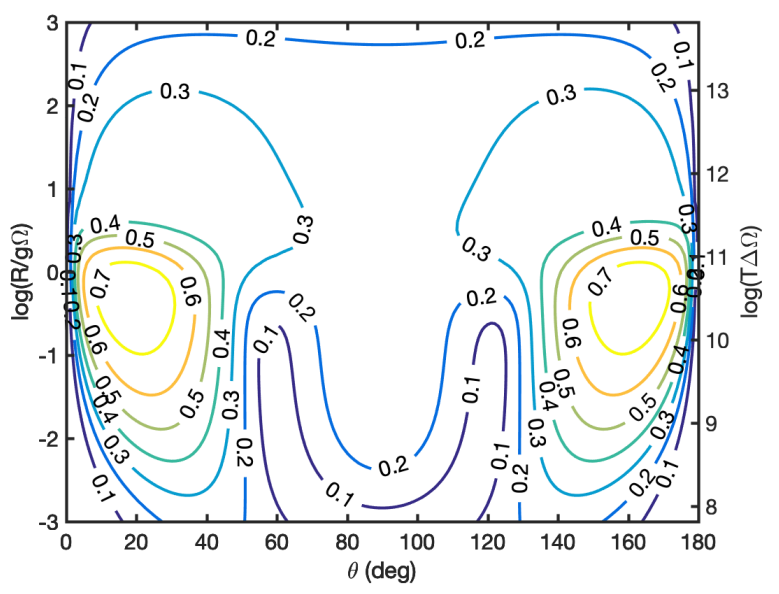

(d)

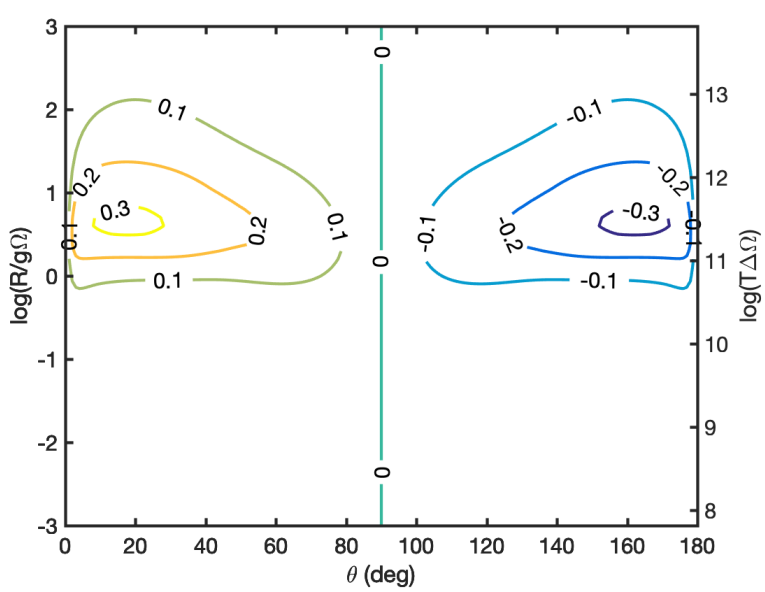

(f)

Fig. A.10. Simulations of $J=1-0 \mathrm{SiO}$ masers with anisotropic pumping direction parallel to the magnetic field. Linear polarization fraction (a,d) and angle (b,e), and circular polarization fraction (c,f). Magnetic field strengths are $B=100 \mathrm{mG}$ for $(\mathrm{a}, \mathrm{b}, \mathrm{c})$ and $B=10 \mathrm{G}$ for $(\mathrm{d}, \mathrm{e}, \mathrm{f})$. 


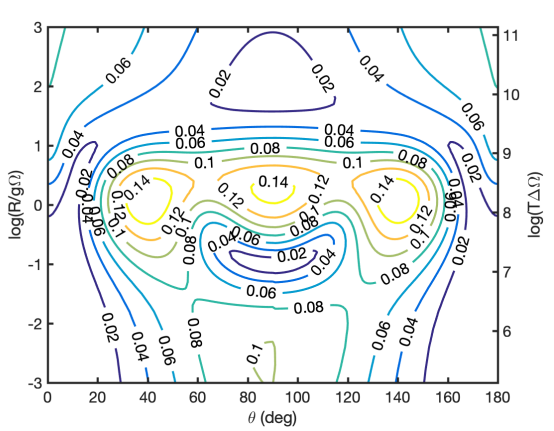

(a)

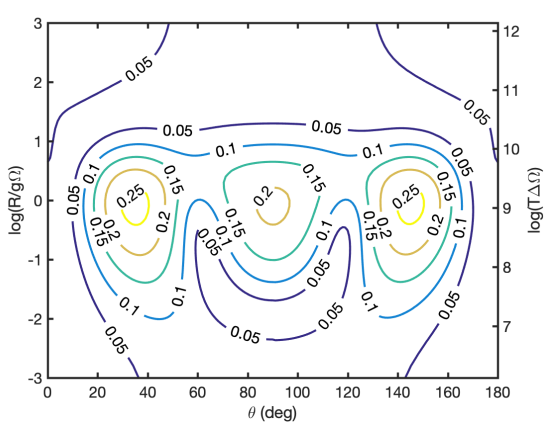

(d)

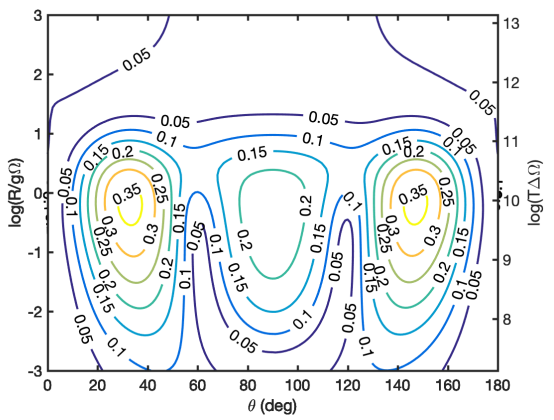

(g)

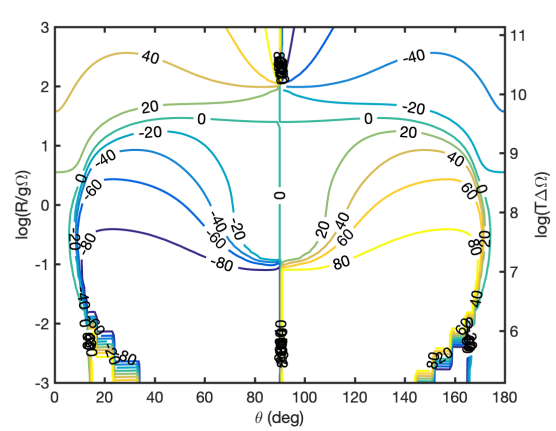

(b)

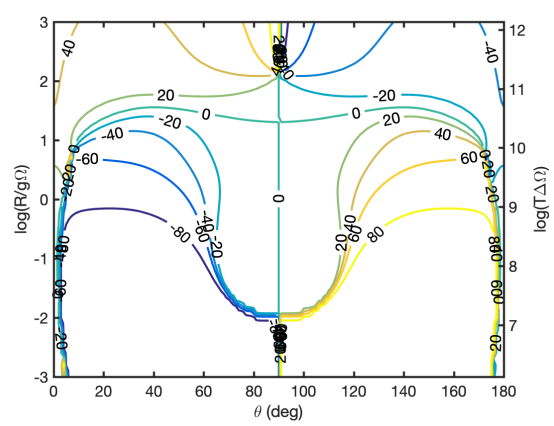

(e)

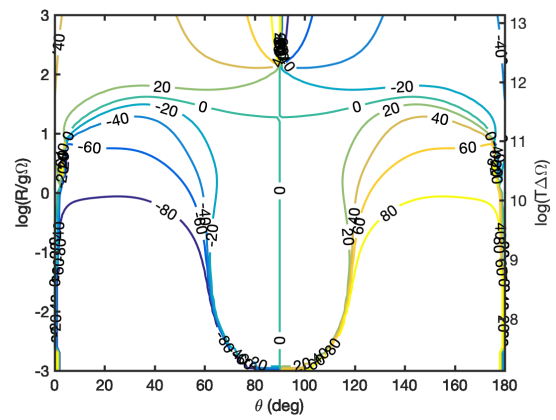

(h)

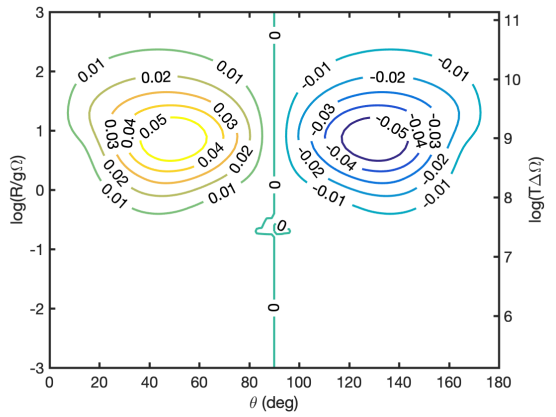

(c)

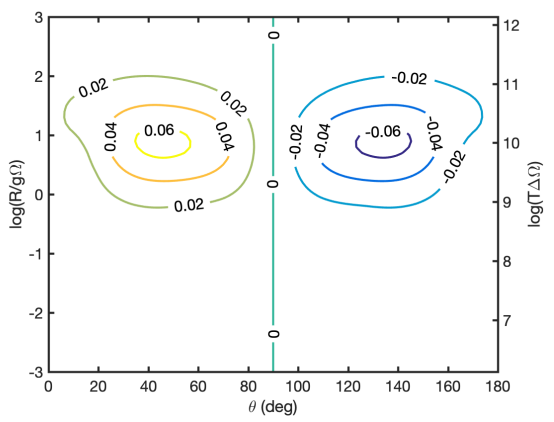

(f)

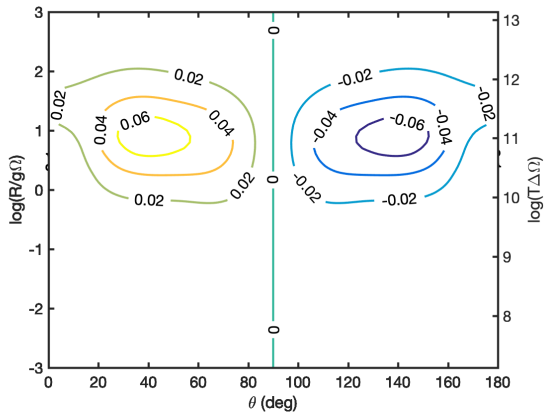

(i)

Fig. A.11. Simulations of $J=2-1 \mathrm{SiO}$ masers with anisotropic pumping direction parallel to the magnetic field. Linear polarization fraction (a,d,f) and angle $(\mathrm{b}, \mathrm{e}, \mathrm{h})$, and circular polarization fraction $(\mathrm{c}, \mathrm{f}, \mathrm{g})$. Magnetic field strengths are $B=100 \mathrm{mG}$ for $(\mathrm{a}, \mathrm{b}, \mathrm{c}), B=1 \mathrm{G}$ for $(\mathrm{d}, \mathrm{e}, \mathrm{f})$, and $B=10 \mathrm{G}$ for $(\mathrm{g}, \mathrm{h}, \mathrm{i})$. 


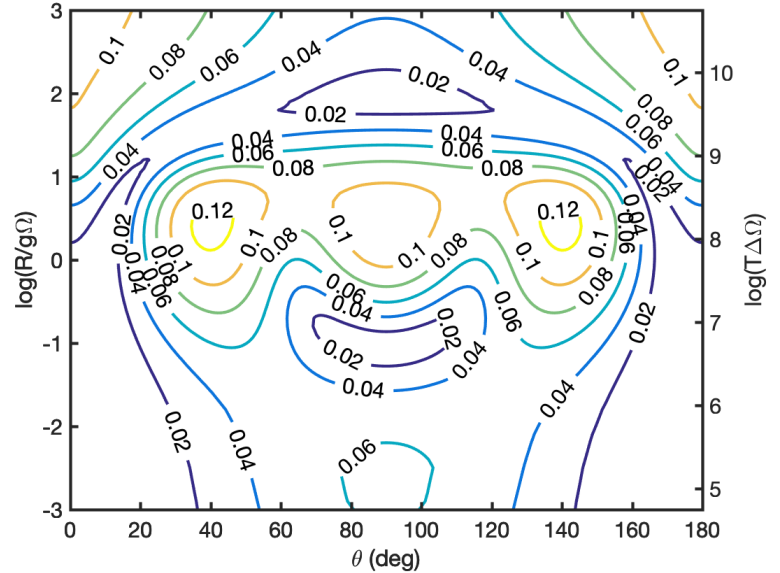

(a)

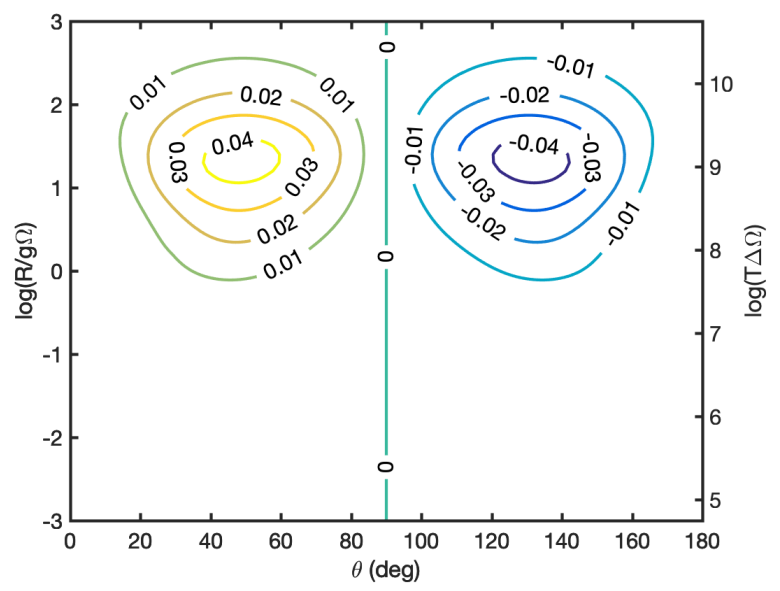

(c)

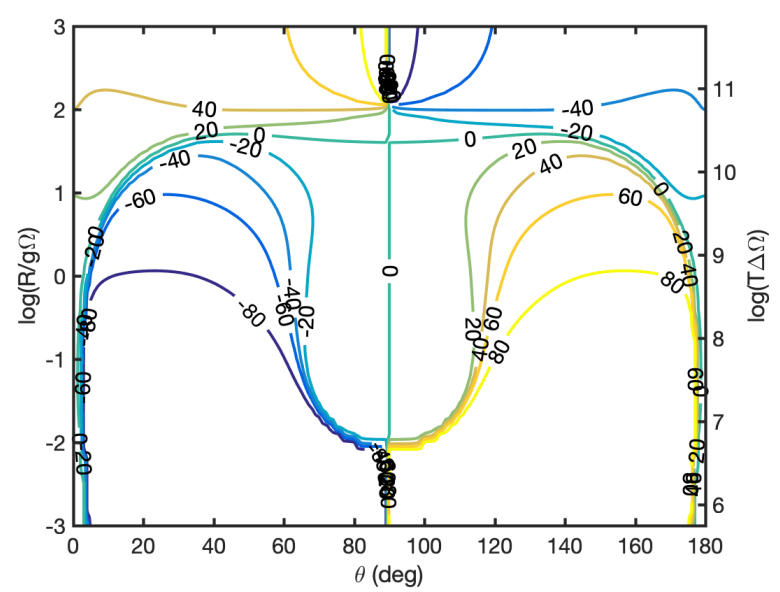

(e)

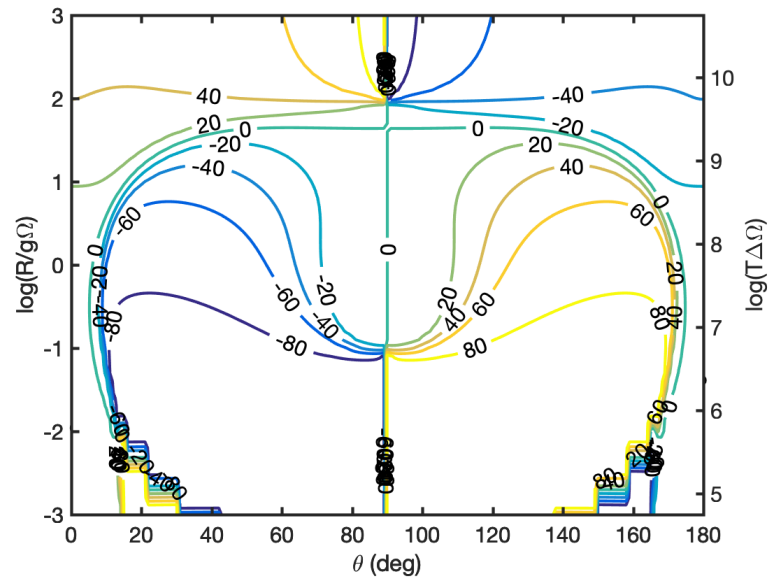

(b)

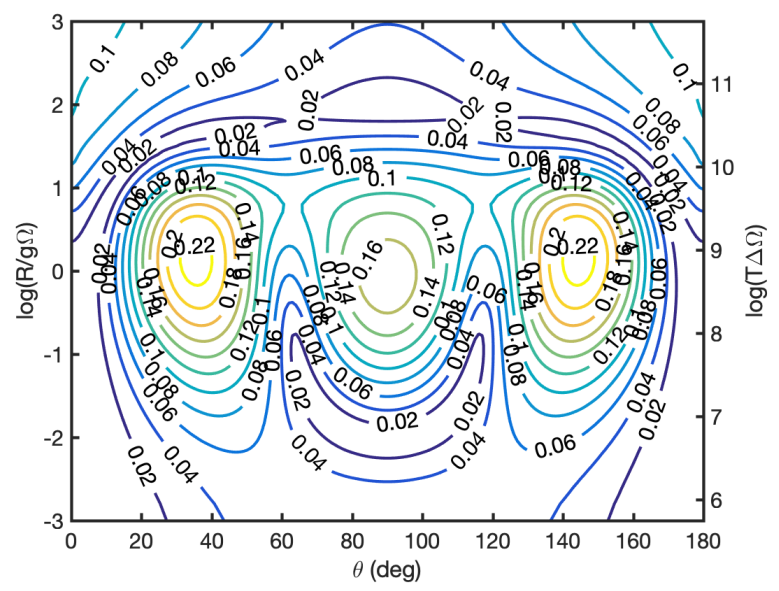

(d)

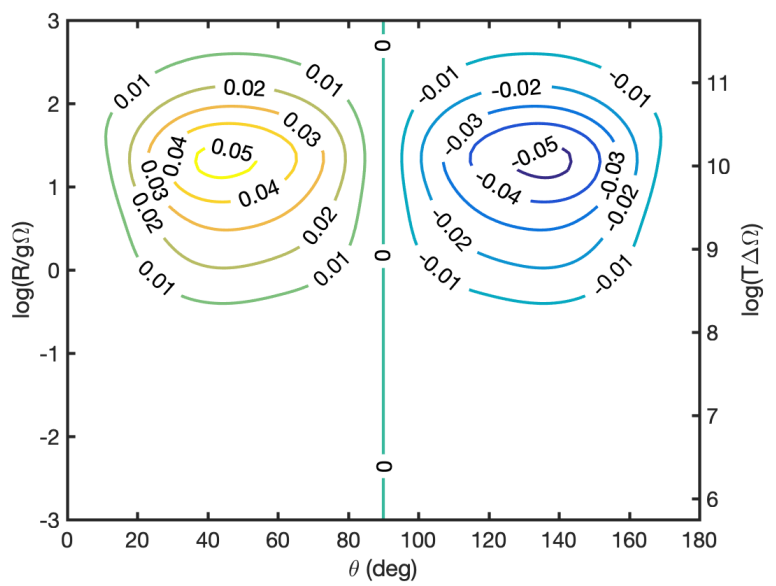

(f)

Fig. A.12. Simulations of $J=3-2 \mathrm{SiO}$ masers with anisotropic pumping direction parallel to the magnetic field. Linear polarization fraction (a,d) and angle (b,e), and circular polarization fraction (c,f). Magnetic field strengths are $B=100 \mathrm{mG}$ for $(\mathrm{a}, \mathrm{b}, \mathrm{c})$ and $B=1 \mathrm{G}$ for $(\mathrm{d}, \mathrm{e}, \mathrm{f})$. 


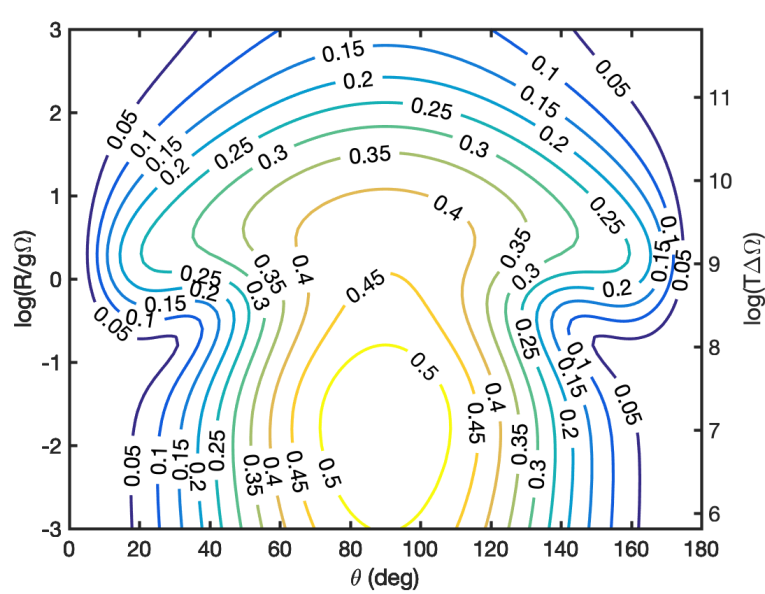

(a)

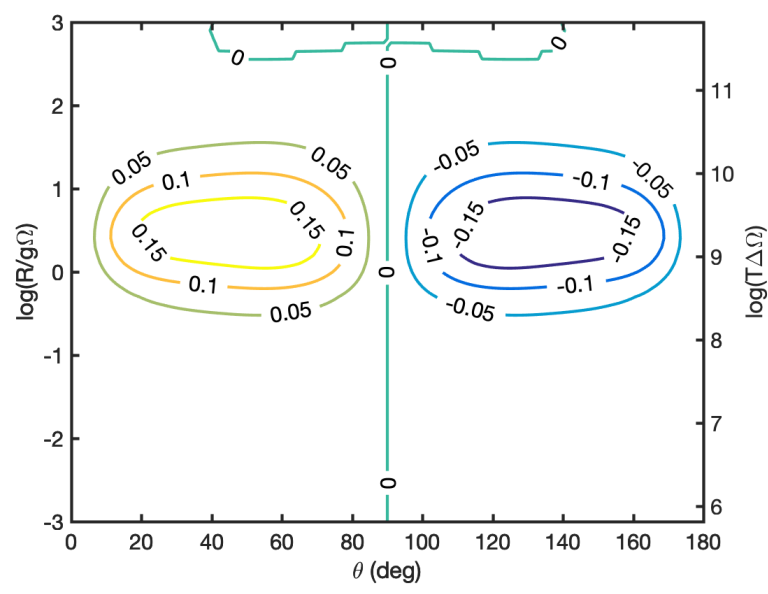

(c)

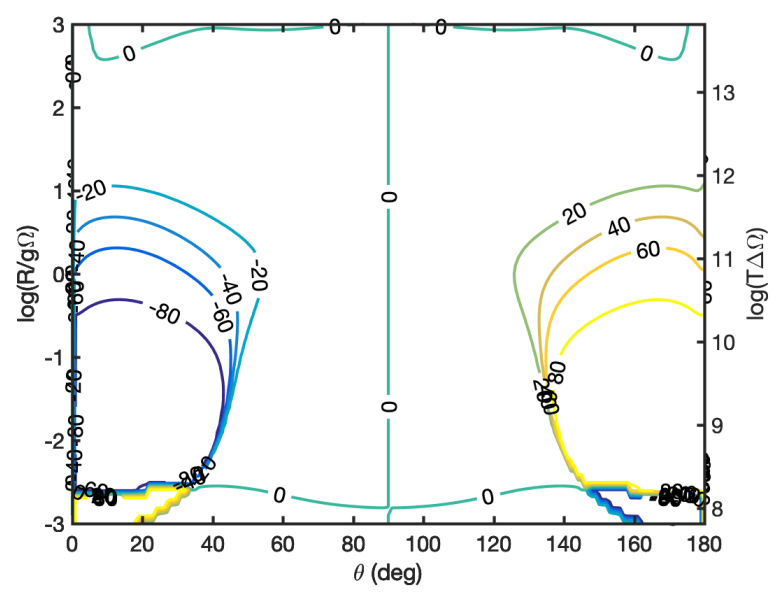

(e)

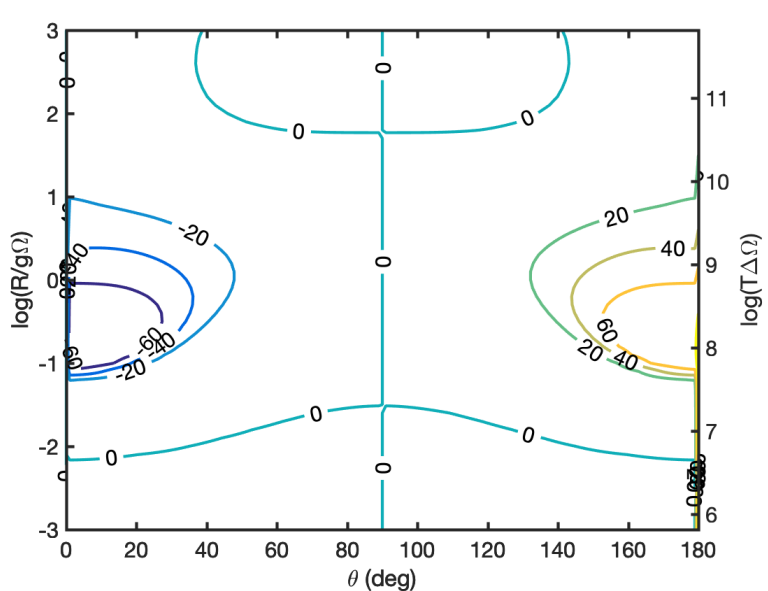

(b)

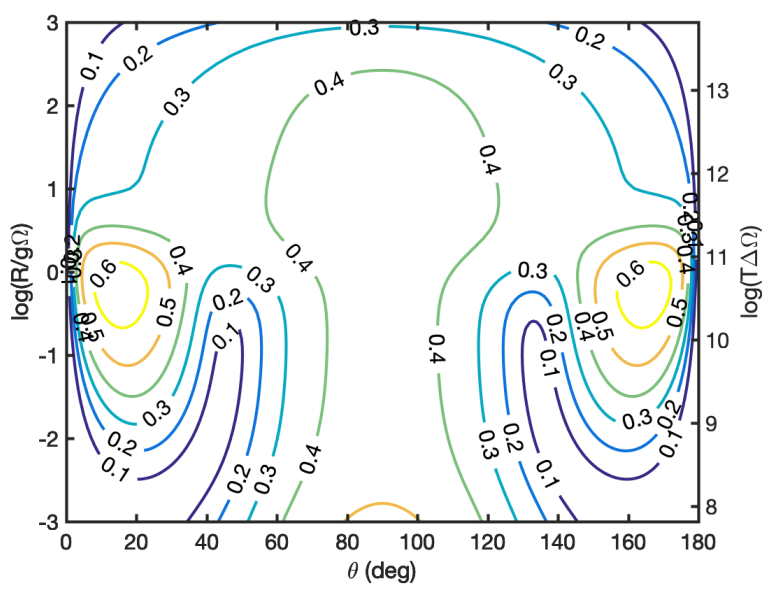

(d)

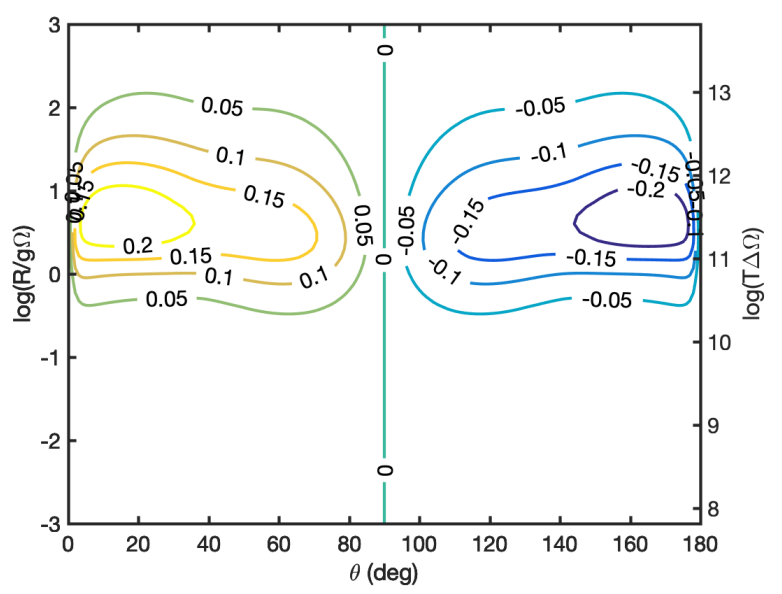

(f)

Fig. A.13. Simulations of $J=1-0 \mathrm{SiO}$ masers with anisotropic pumping direction perpendicular to the magnetic field and propagation direction. Linear polarization fraction (a,d) and angle (b,e), and circular polarization fraction (c,f). Magnetic field strengths are $B=100 \mathrm{mG}$ for $(\mathrm{a}, \mathrm{b}, \mathrm{c})$ and $B=10 \mathrm{G}$ for $(\mathrm{d}, \mathrm{e}, \mathrm{f})$. 


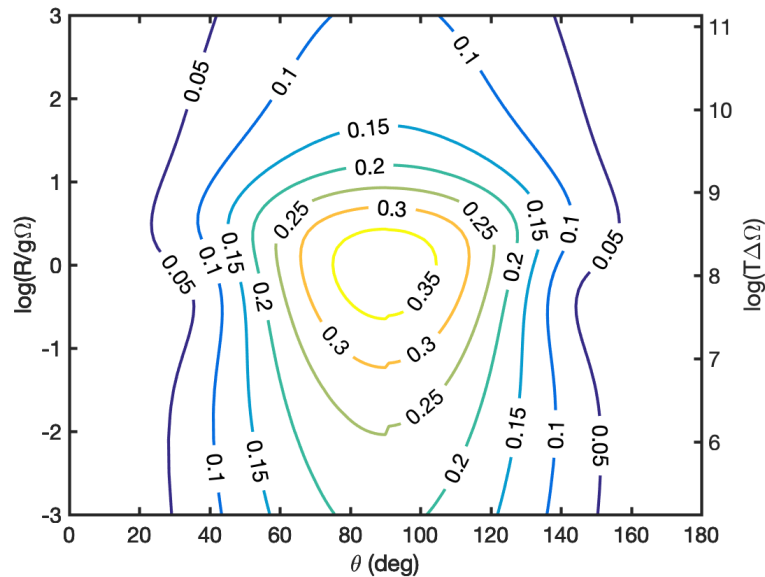

(a)

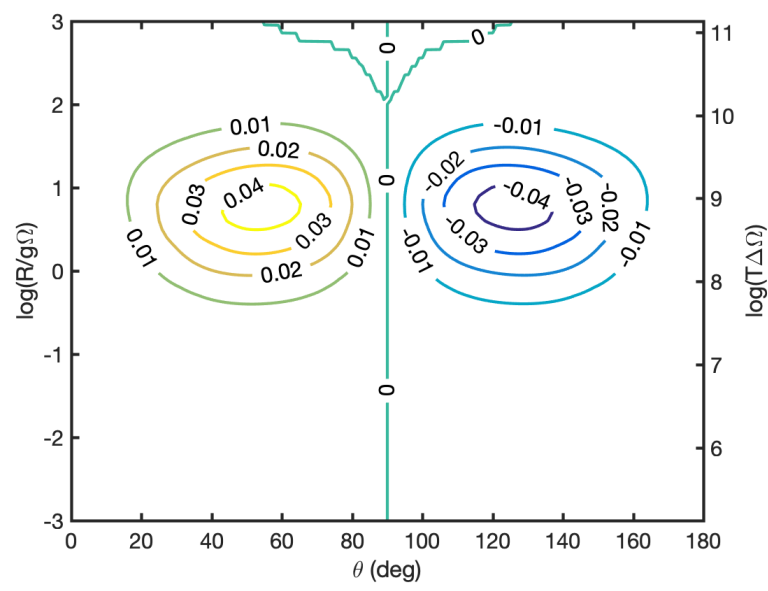

(c)

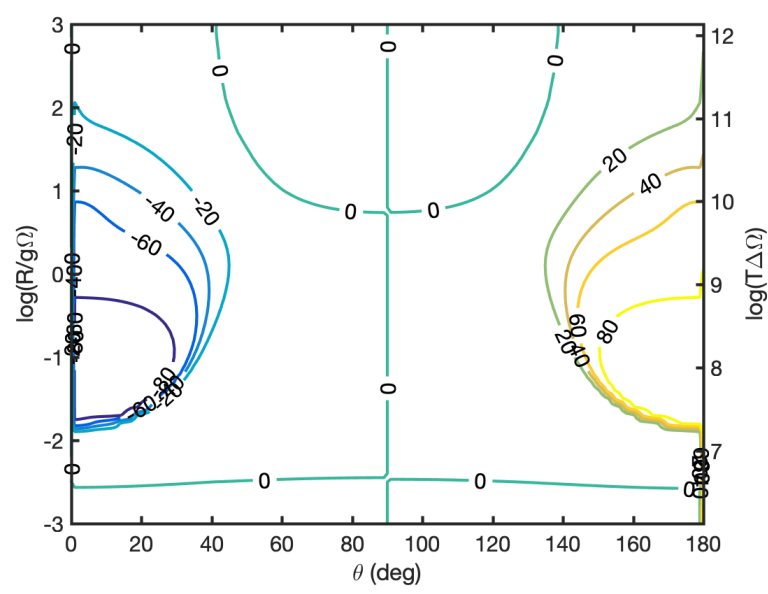

(e)

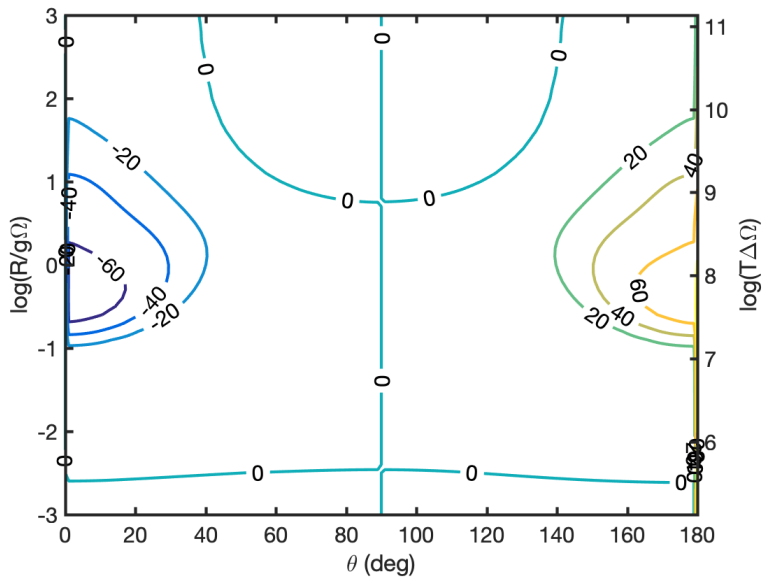

(b)

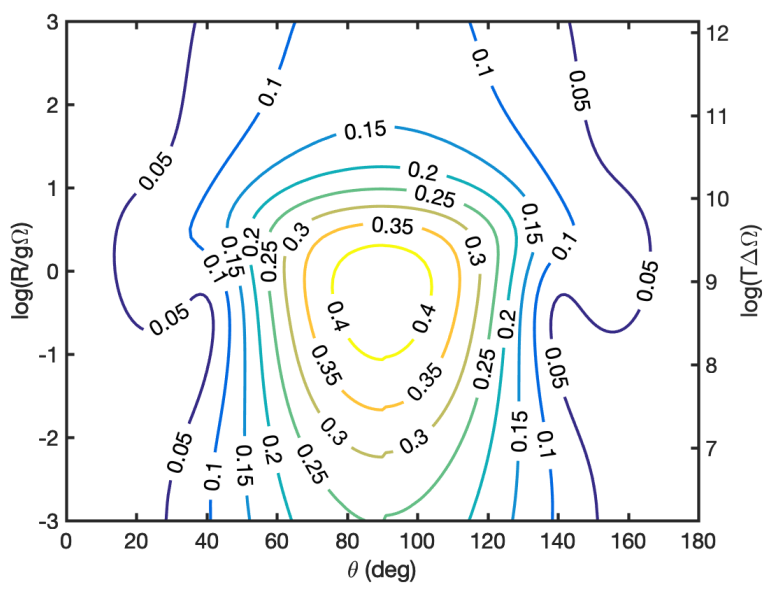

(d)

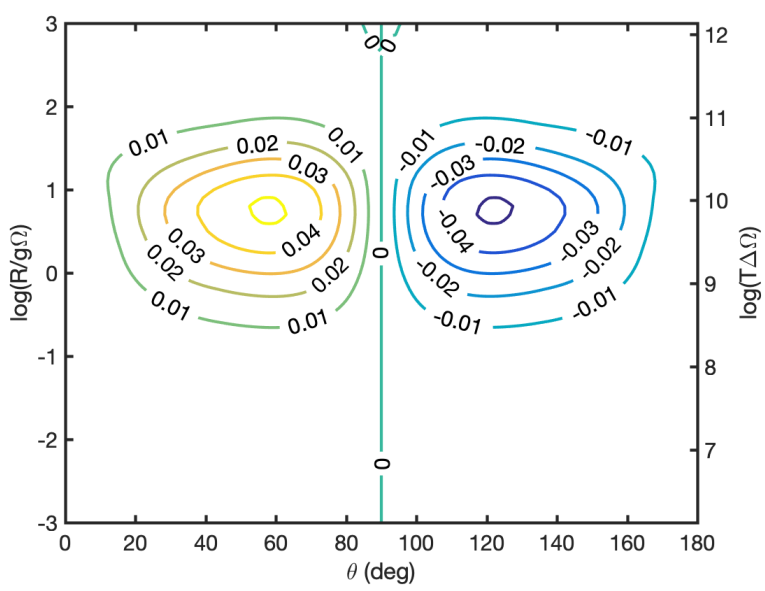

(f)

Fig. A.14. Simulations of $J=2-1 \mathrm{SiO}$ masers with anisotropic pumping direction perpendicular to the magnetic field and propagation direction. Linear polarization fraction (a,d) and angle (b,e), and circular polarization fraction (c,f). Magnetic field strengths are $B=100 \mathrm{mG}$ for $(\mathrm{a}, \mathrm{b}, \mathrm{c})$ and $B=1 \mathrm{G}$ for $(\mathrm{d}, \mathrm{e}, \mathrm{f})$. 


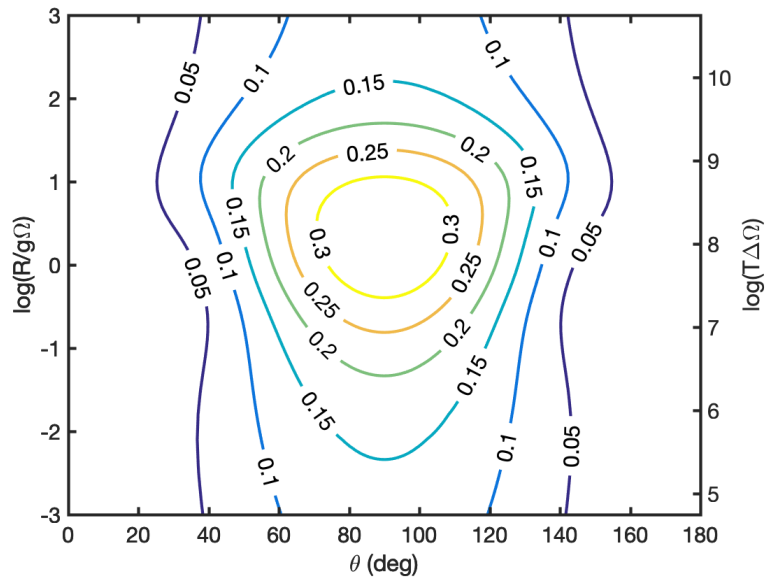

(a)

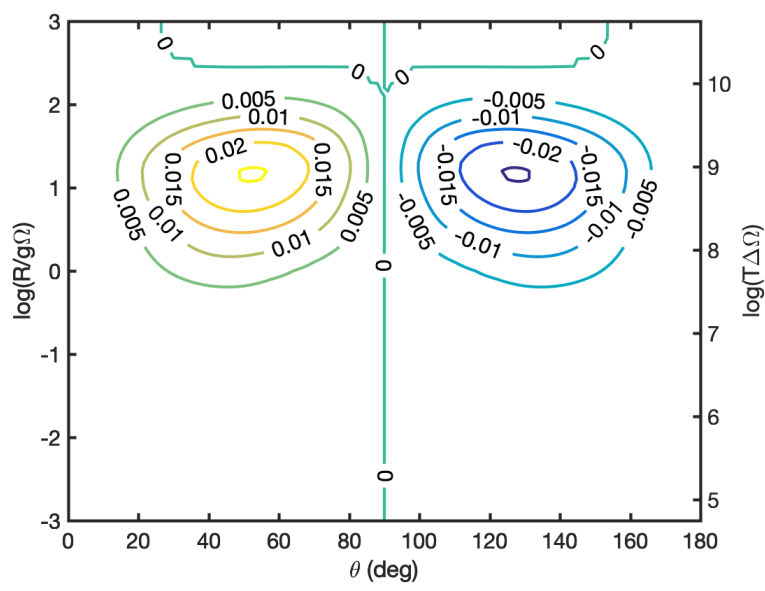

(c)

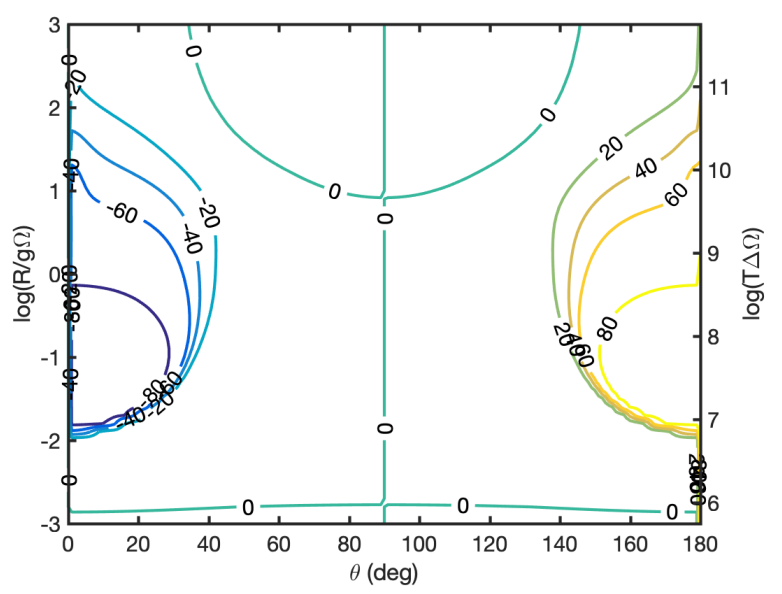

(e)

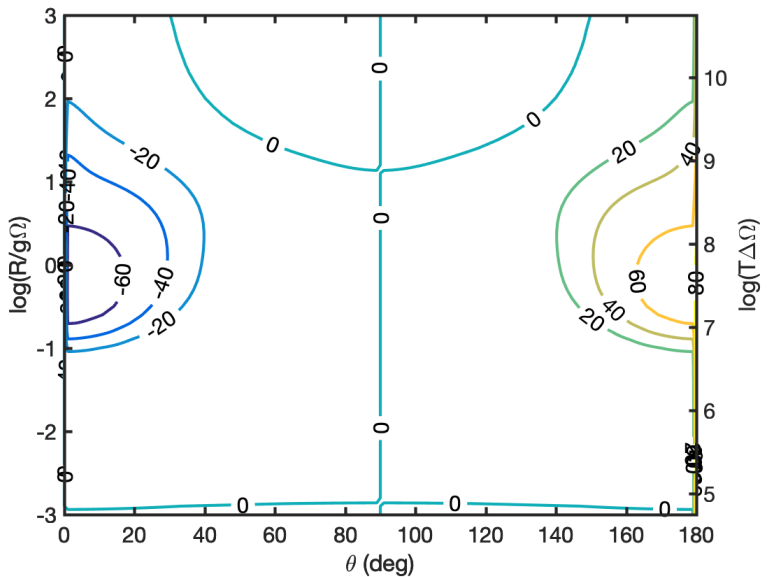

(b)

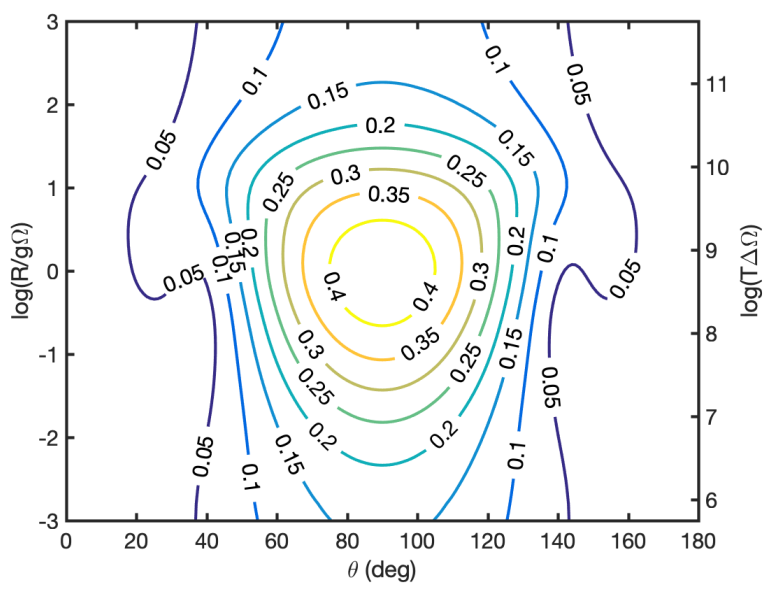

(d)

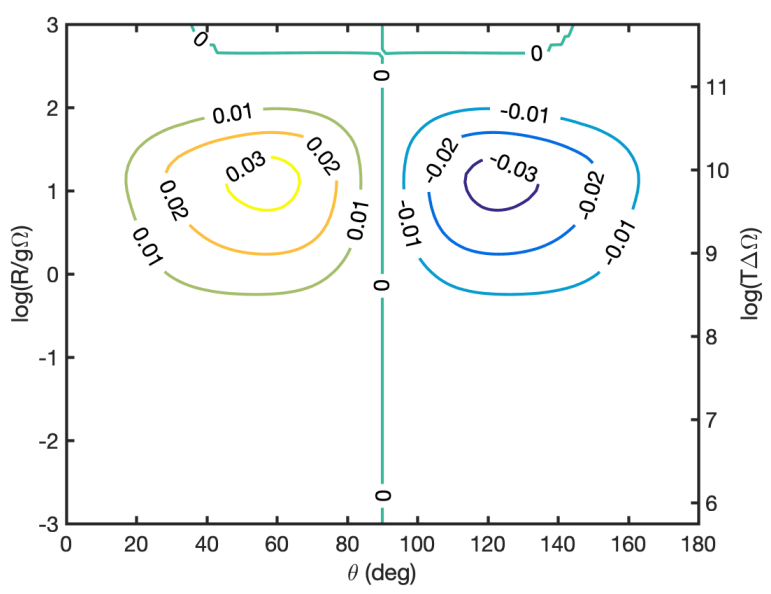

(f)

Fig. A.15. Simulations of $J=3-2 \mathrm{SiO}$ masers with anisotropic pumping direction perpendicular to the magnetic field and propagation direction. Linear polarization fraction (a,d) and angle (b,e), and circular polarization fraction (c,f). Magnetic field strengths are $B=100 \mathrm{mG}$ for $(\mathrm{a}, \mathrm{b}, \mathrm{c})$ and $B=1 \mathrm{G}$ for $(\mathrm{d}, \mathrm{e}, \mathrm{f})$. 


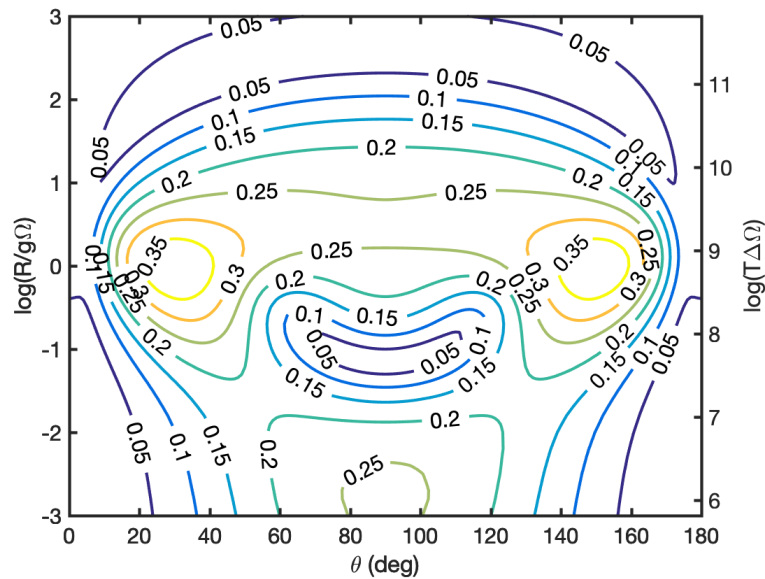

(a)

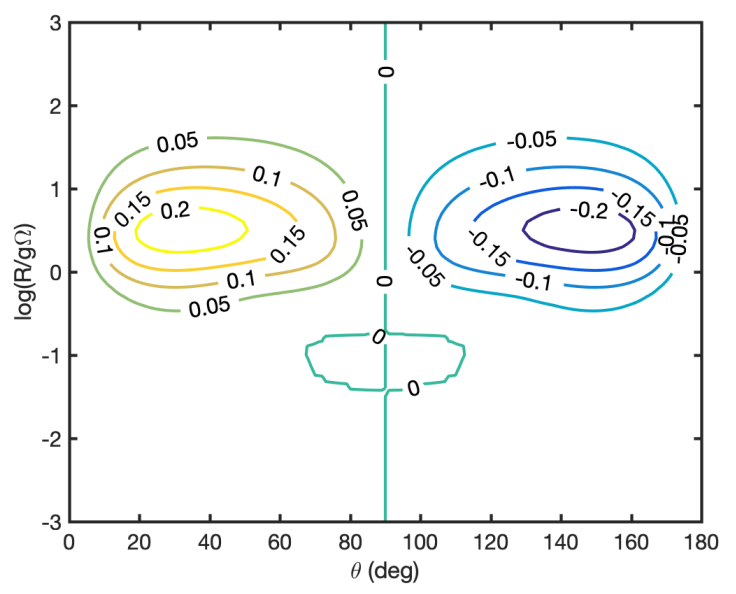

(c)

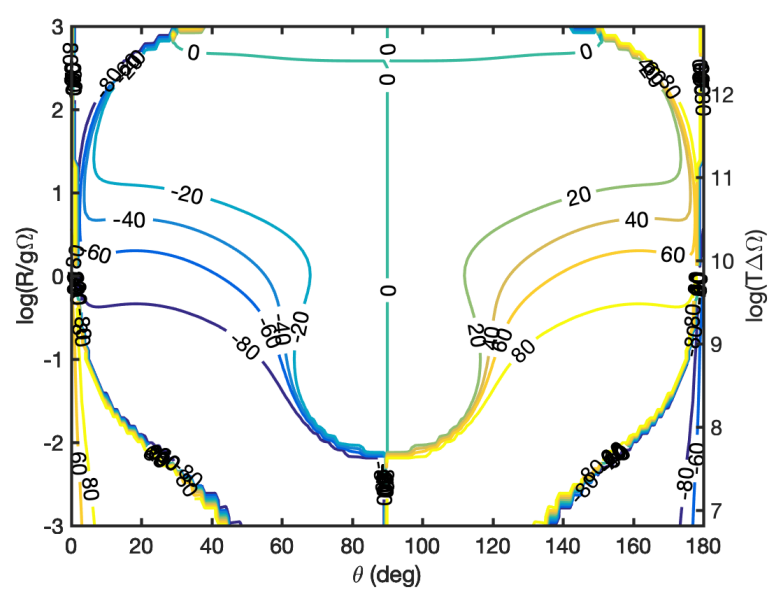

(e)

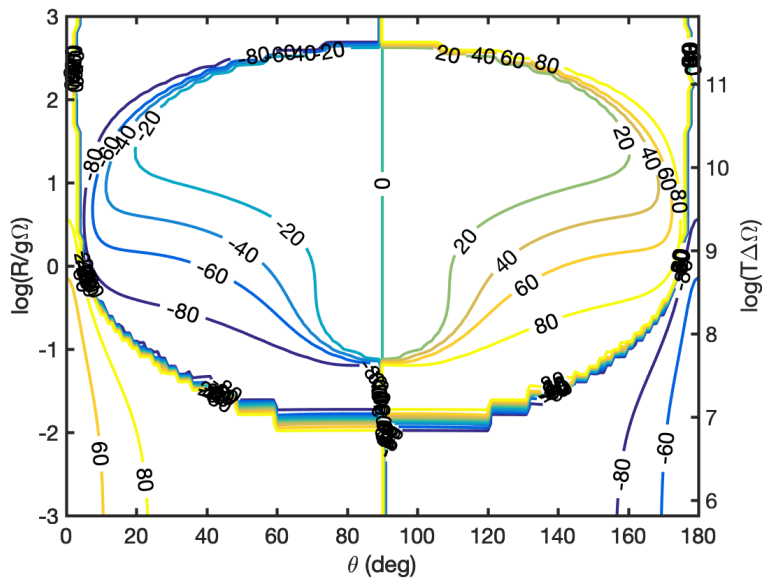

(b)

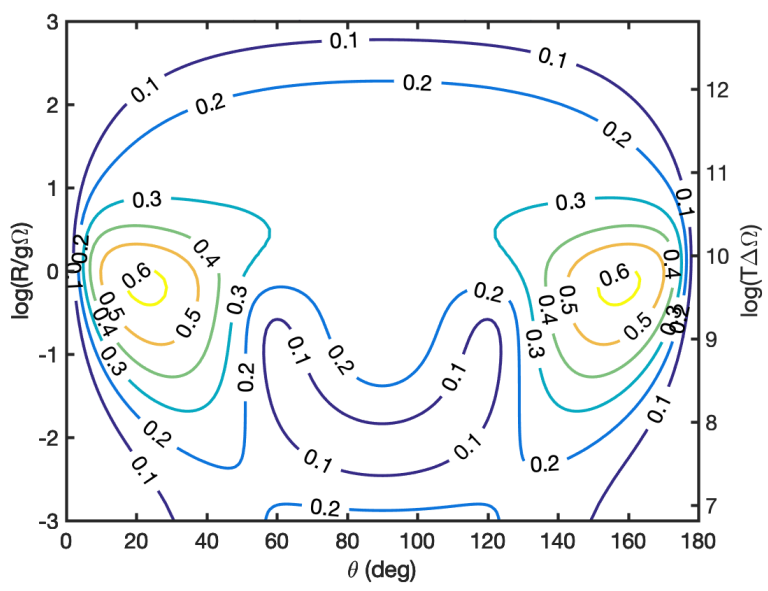

(d)

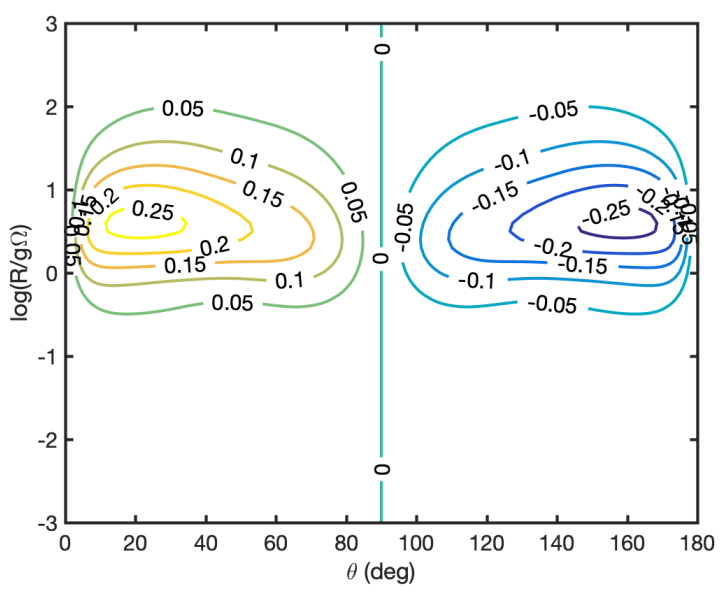

(f)

Fig. A.16. Simulations of $J=1-0 \mathrm{SiO}$ masers with anisotropic pumping direction at $45^{\circ}$ from the magnetic field in the plane perpendicular to the propagation direction. Linear polarization fraction $(\mathrm{a}, \mathrm{d})$ and angle $(\mathrm{b}, \mathrm{e})$, and circular polarization fraction (c,f). Magnetic field strengths are $B=100 \mathrm{mG}$ for $(\mathrm{a}, \mathrm{b}, \mathrm{c})$ and $B=1 \mathrm{G}$ for $(\mathrm{d}, \mathrm{e}, \mathrm{f})$. 


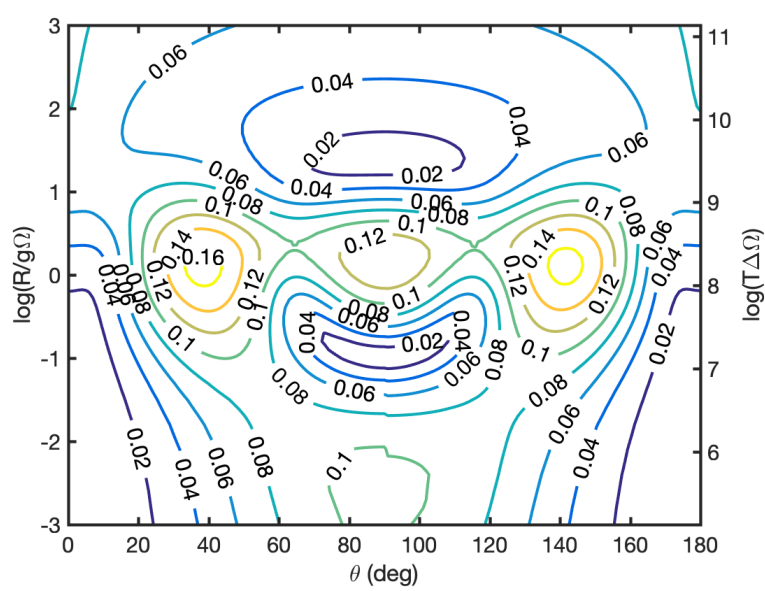

(a)

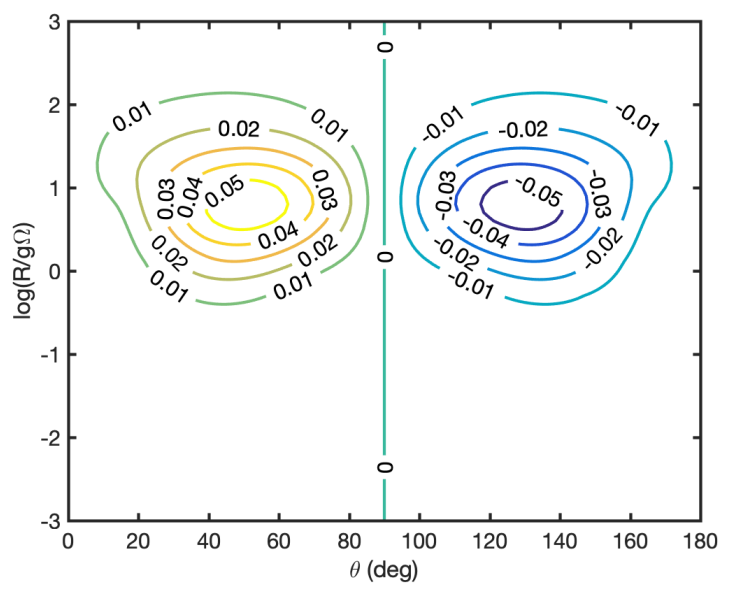

(c)

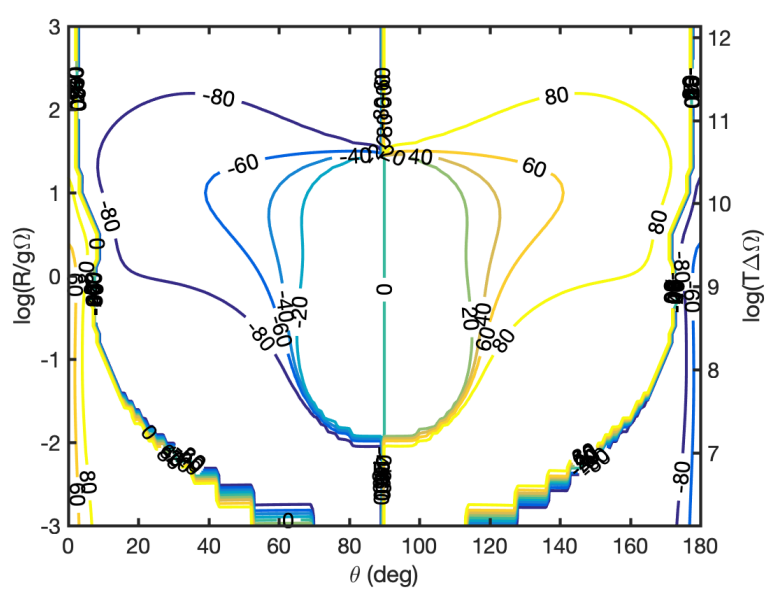

(e)

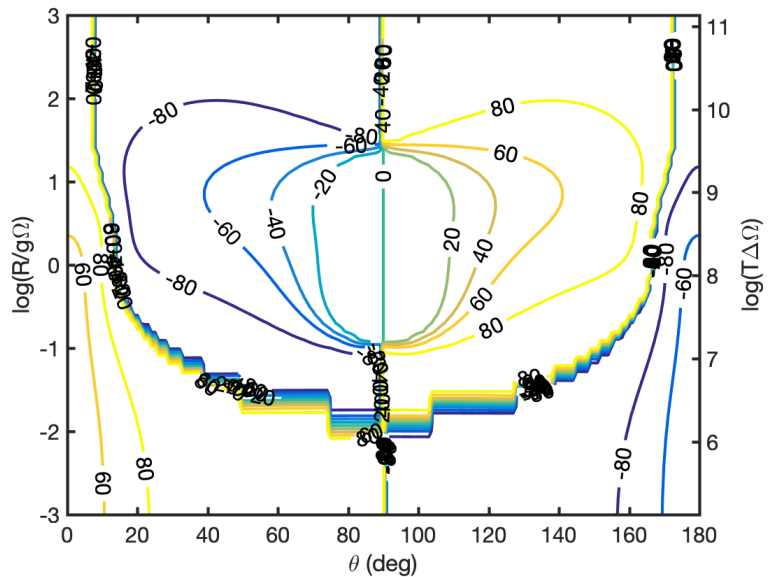

(b)

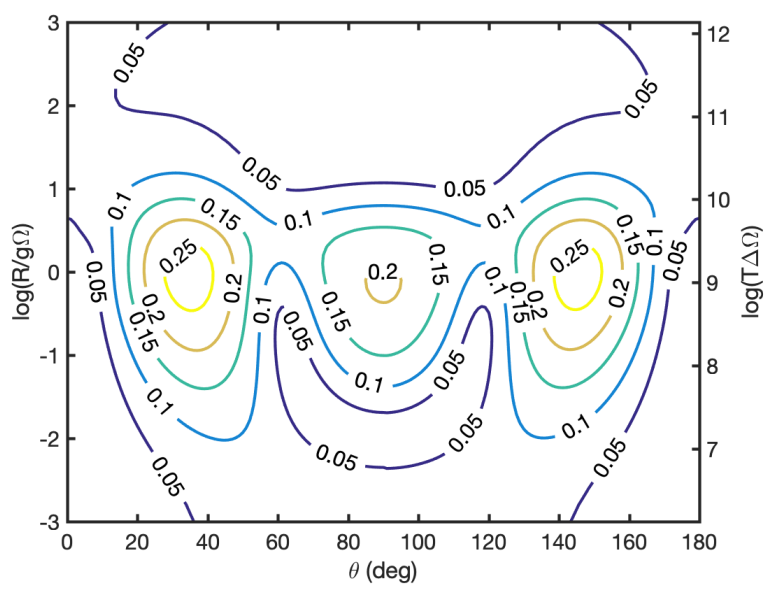

(d)

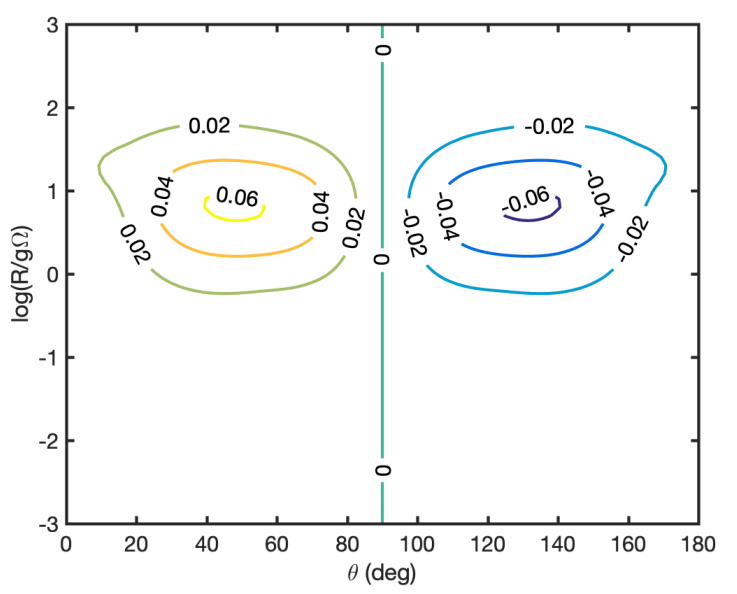

(f)

Fig. A.17. Simulations of $J=2-1 \mathrm{SiO}$ masers with anisotropic pumping direction at $45^{\circ}$ from the magnetic field in the plane perpendicular to the propagation direction. Linear polarization fraction $(a, d)$ and angle $(b, e)$, and circular polarization fraction $(c, f)$. Magnetic field strengths are $B=100 \mathrm{mG}$ for $(\mathrm{a}, \mathrm{b}, \mathrm{c})$ and $B=1 \mathrm{G}$ for $(\mathrm{d}, \mathrm{e}, \mathrm{f})$. 


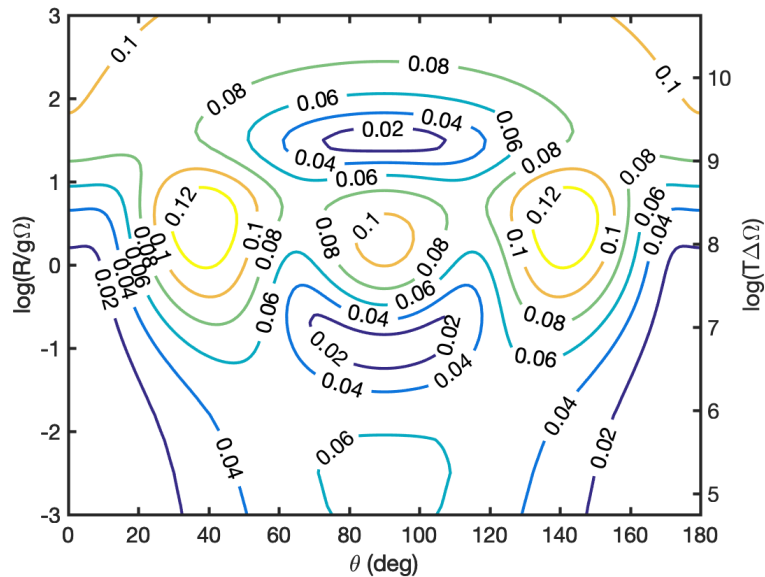

(a)

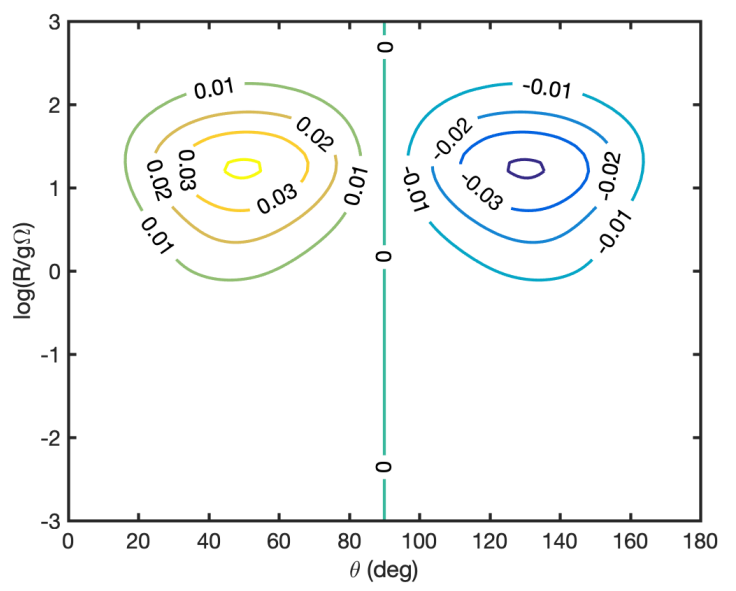

(c)

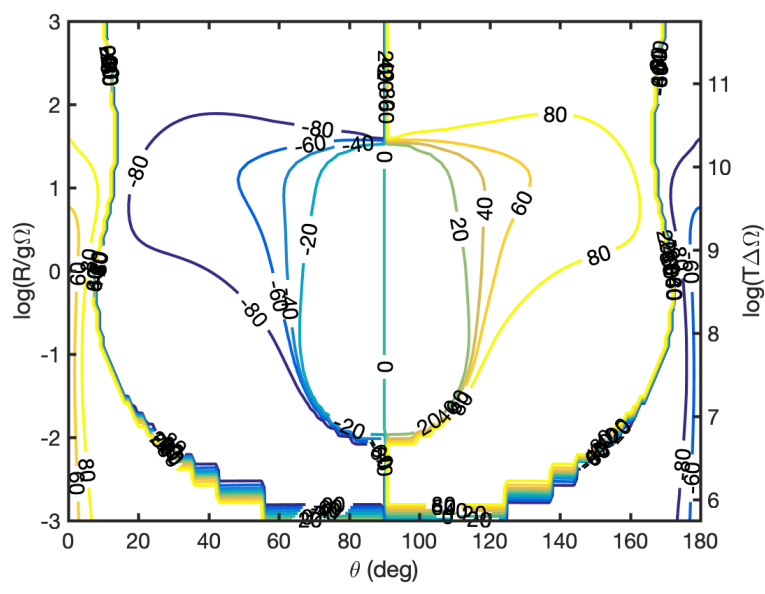

(e)

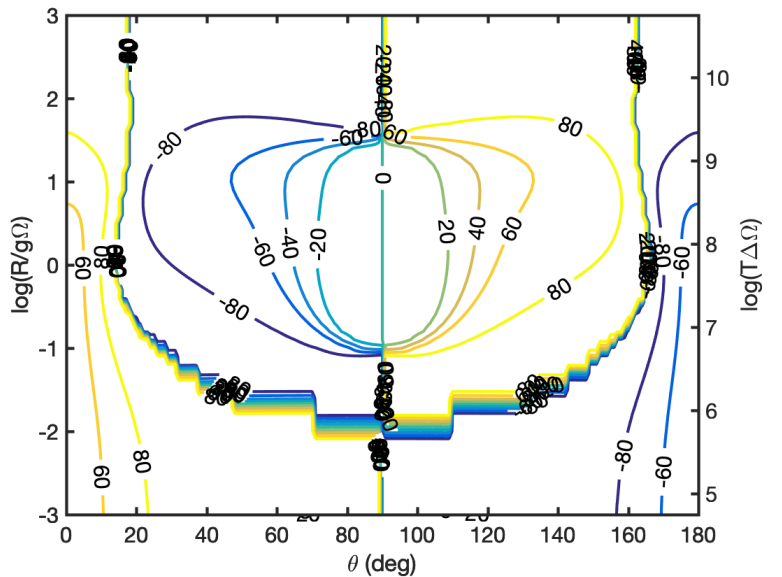

(b)

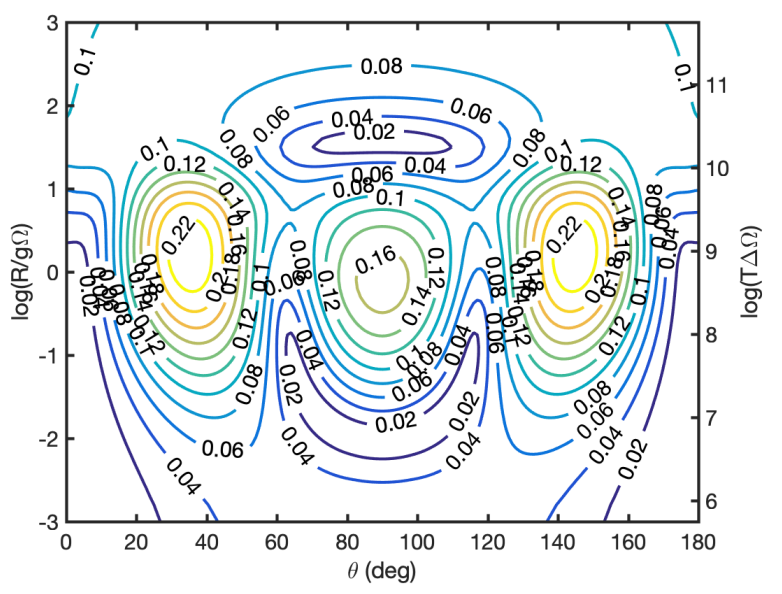

(d)

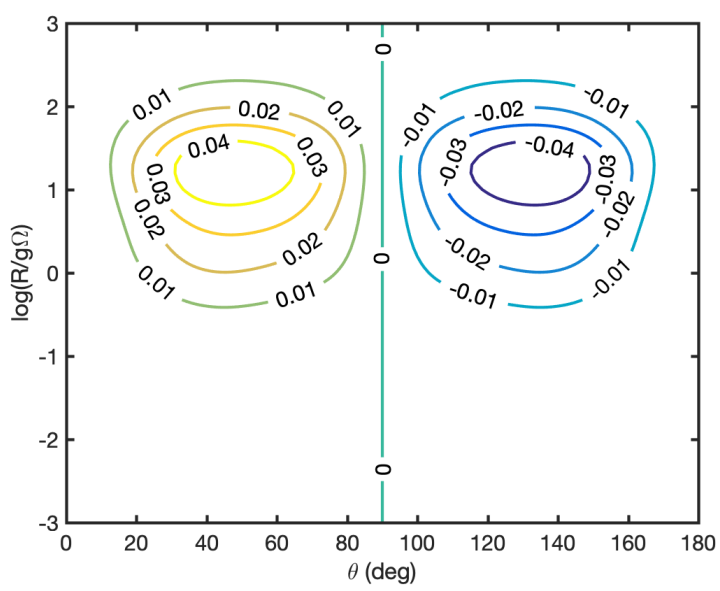

(f)

Fig. A.18. Simulations of $J=3-2 \mathrm{SiO}$ masers with anisotropic pumping direction at $45^{\circ}$ from the magnetic field in the plane perpendicular to the propagation direction. Linear polarization fraction $(a, d)$ and angle $(b, e)$, and circular polarization fraction $(c, f)$. Magnetic field strengths are $B=100 \mathrm{mG}$ for $(\mathrm{a}, \mathrm{b}, \mathrm{c})$ and $B=1 \mathrm{G}$ for $(\mathrm{d}, \mathrm{e}, \mathrm{f})$. 
A\&A 628, A14 (2019)

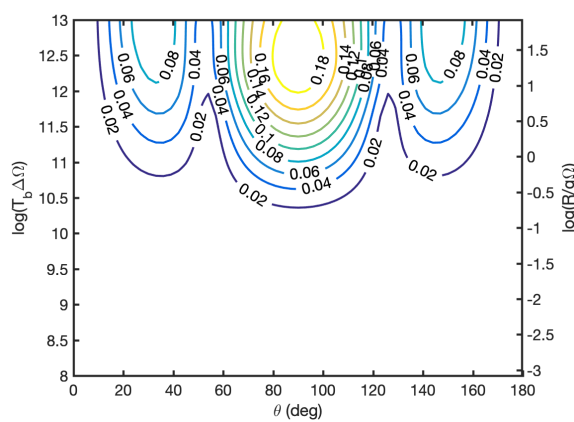

(a)

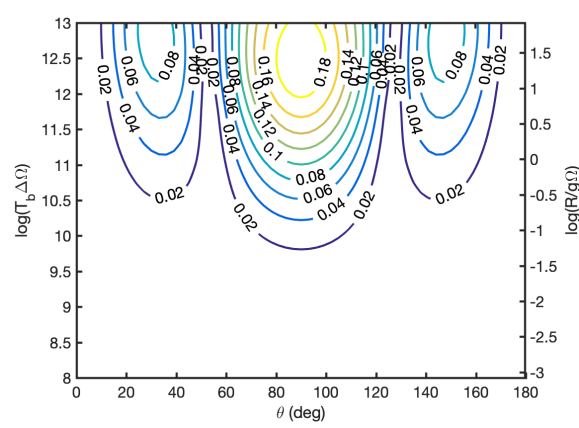

(d)

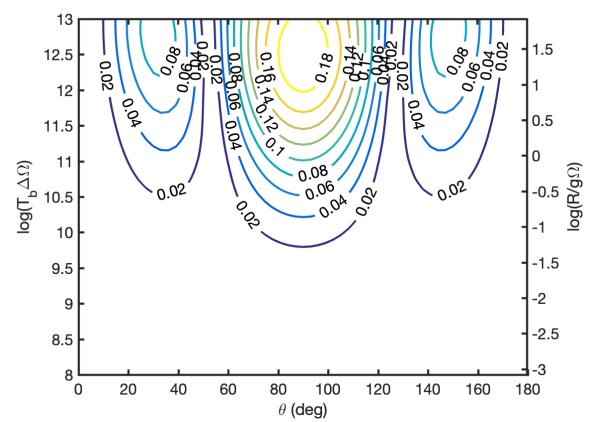

(g)

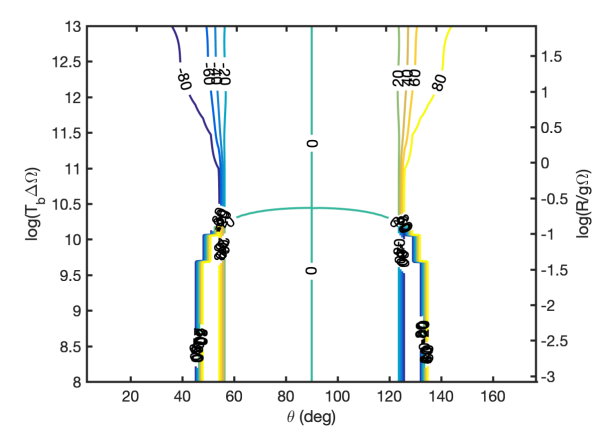

(b)

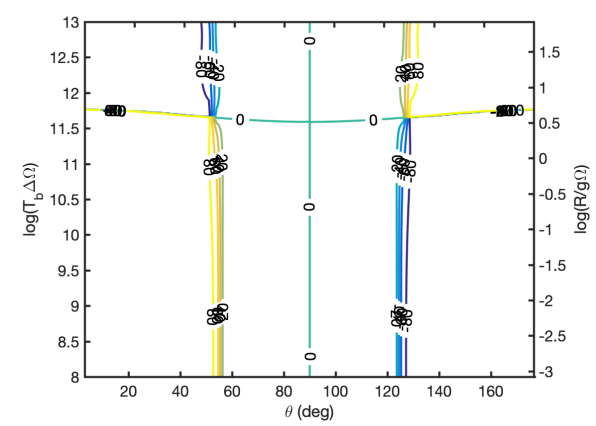

(e)

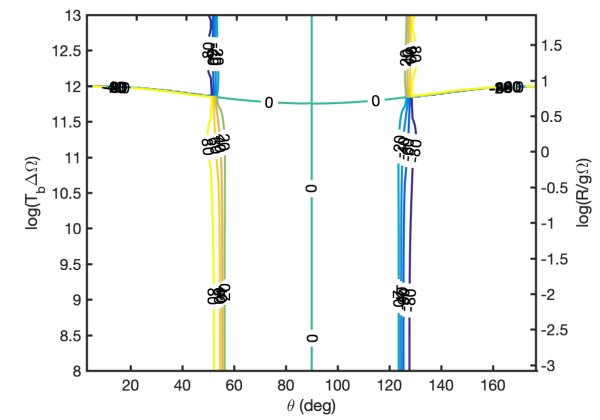

(h)

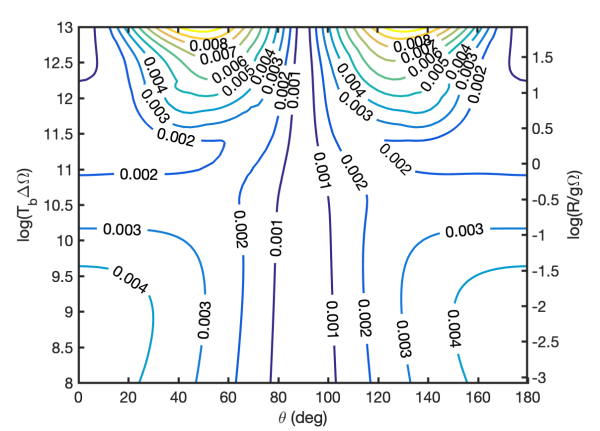

(c)

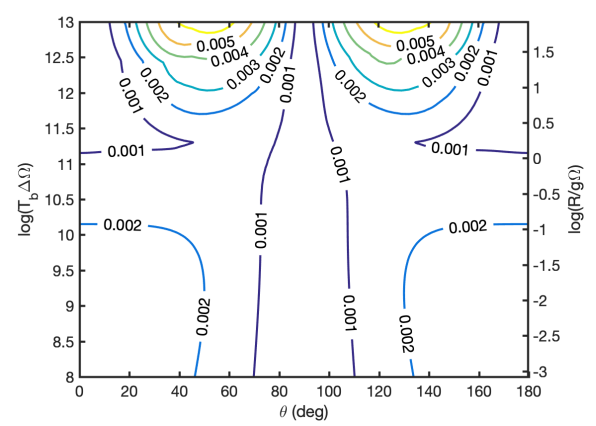

(f)

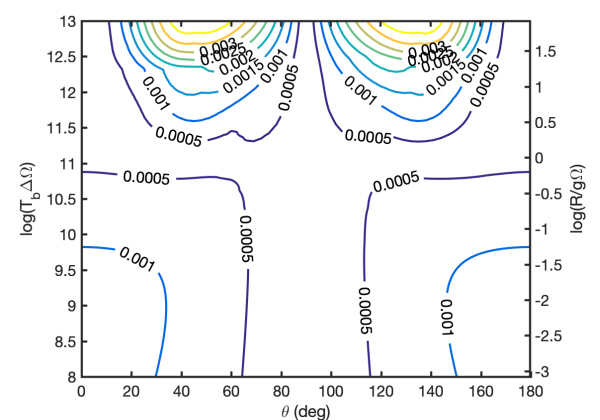

(i)

Fig. A.19. Polarization of a water maser isotropically pumped at $B=20 \mathrm{mG}$. Linear polarization fraction (a,d,g) and angle (b,e,h), and circular polarization fraction (c,f,i). Thermal width used is $v_{\mathrm{th}}=0.6 \mathrm{~km} \mathrm{~s}^{-1}(\mathrm{a}, \mathrm{b}, \mathrm{c}), 1 \mathrm{~km} \mathrm{~s}^{-1}$ (d,e,f), and $2 \mathrm{~km} \mathrm{~s}^{-1}(\mathrm{~g}, \mathrm{~h}, \mathrm{i})$. 
B. Lankhaar and W. Vlemmings: Maser polarization

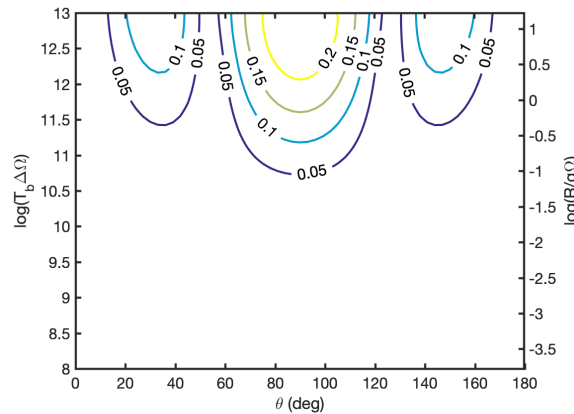

(a)

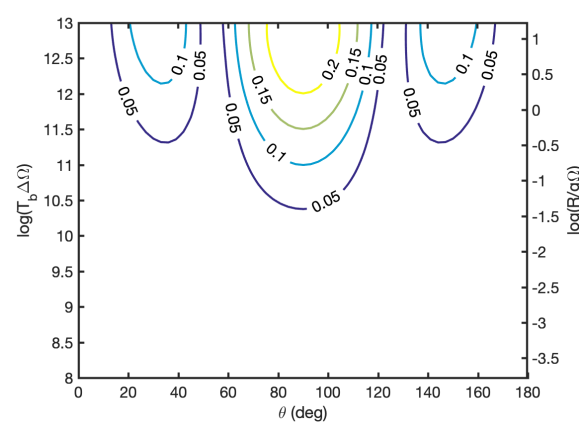

(d)

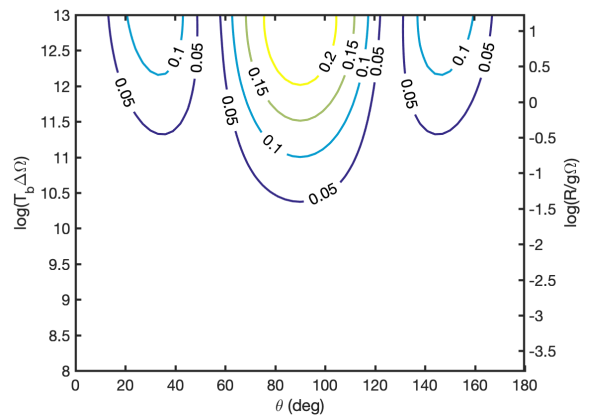

(g)

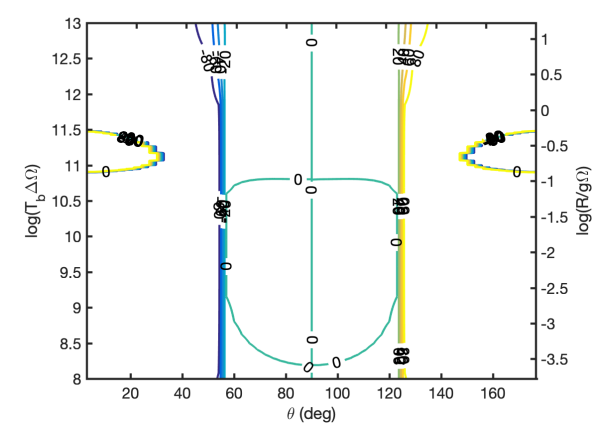

(b)

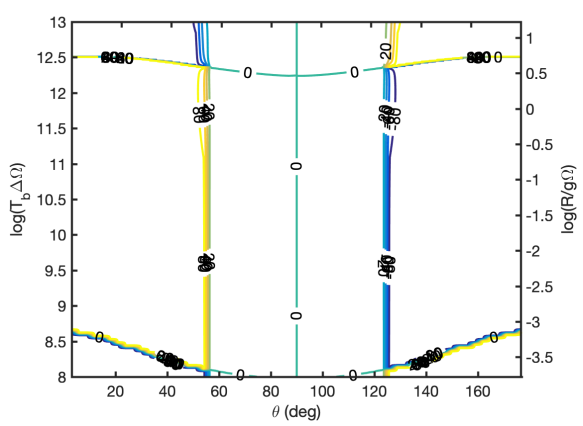

(e)

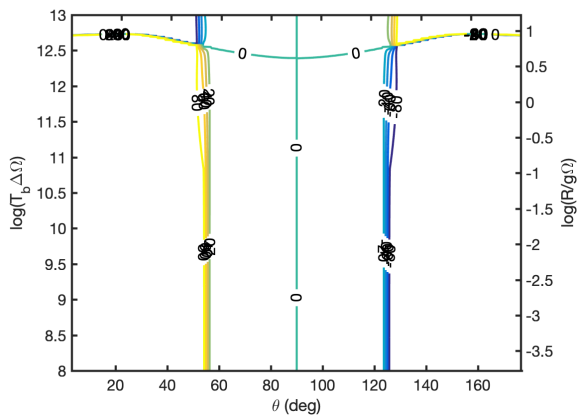

(h)

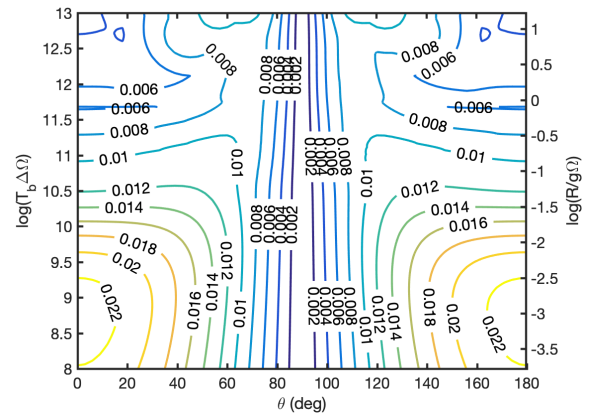

(c)

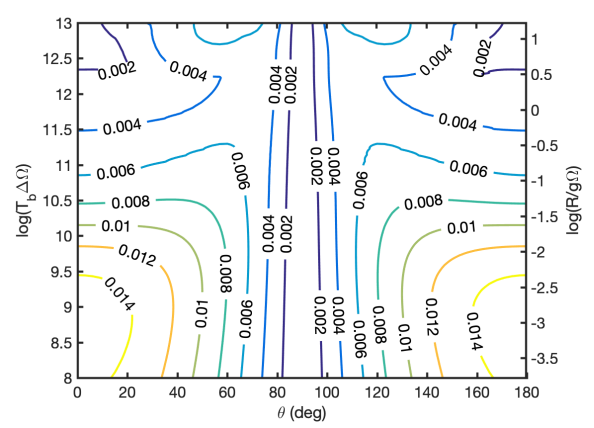

(f)

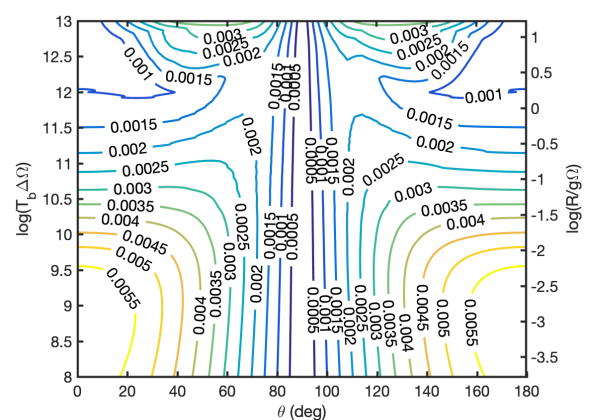

(i)

Fig. A.20. Polarization of a water maser isotropically pumped at $B=100 \mathrm{mG}$. Linear polarization fraction (a,d,g) and angle (b,e,h), and circular polarization fraction (c,f,i). Thermal width used is $v_{\text {th }}=0.6 \mathrm{~km} \mathrm{~s}^{-1}(\mathrm{a}, \mathrm{b}, \mathrm{c}), 1 \mathrm{~km} \mathrm{~s}^{-1}$ (d,e,f), and $2 \mathrm{~km} \mathrm{~s}^{-1}(\mathrm{~g}, \mathrm{~h}, \mathrm{i})$. 


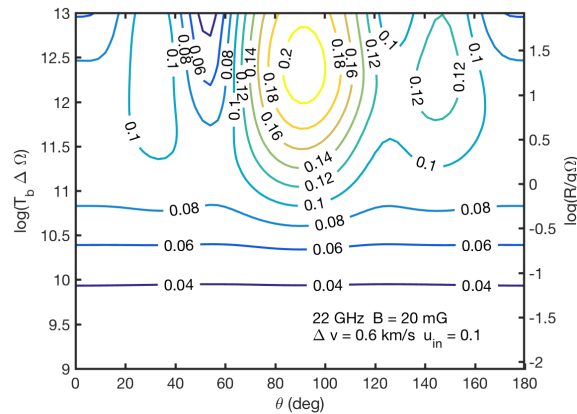

(a)

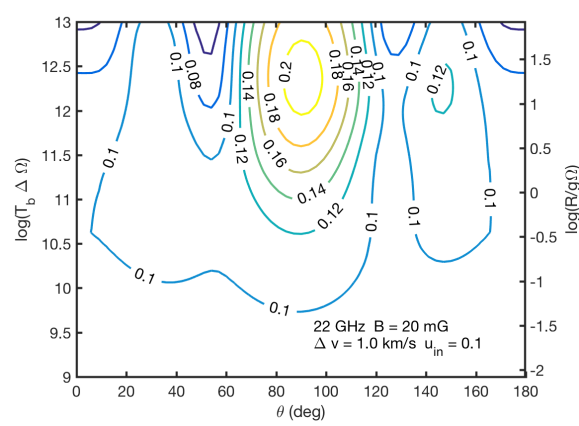

(d)

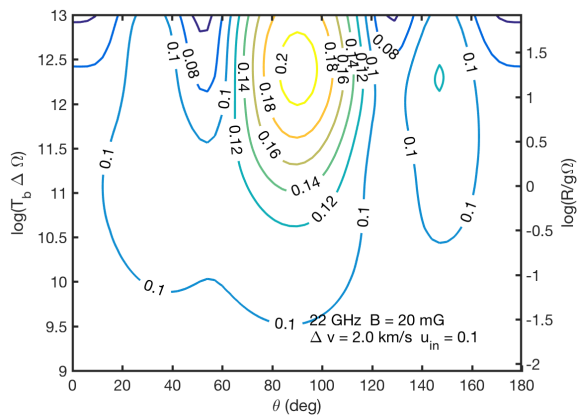

(g)

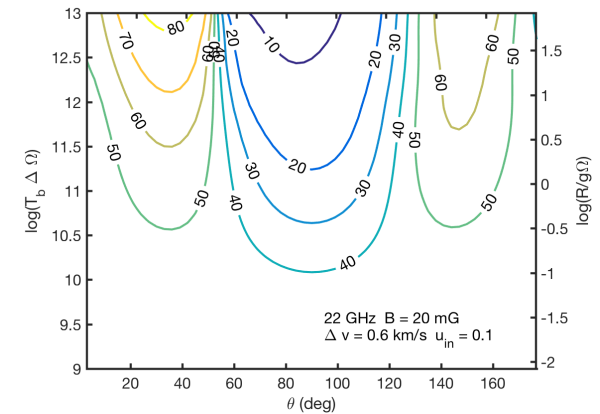

(b)

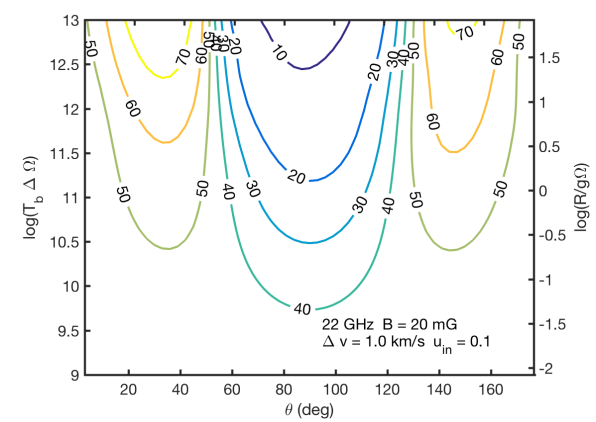

(e)

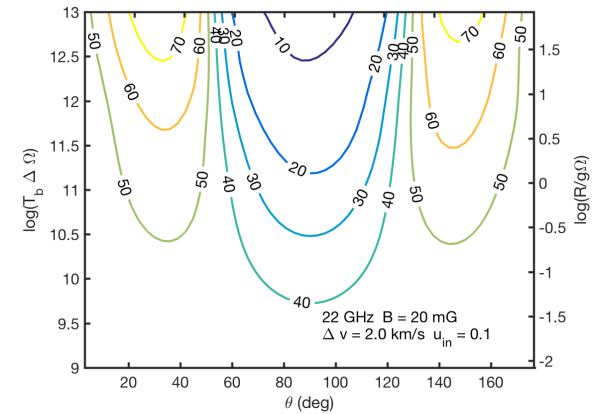

(h)

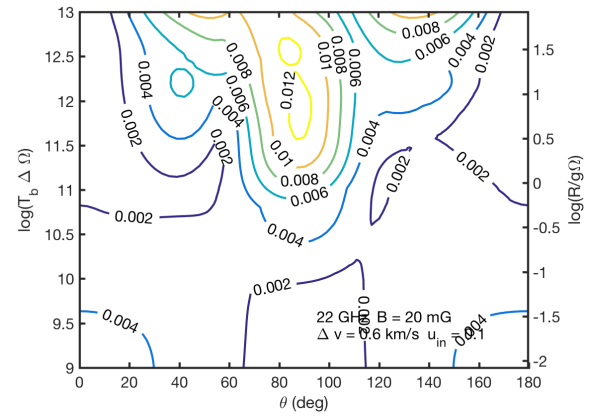

(c)

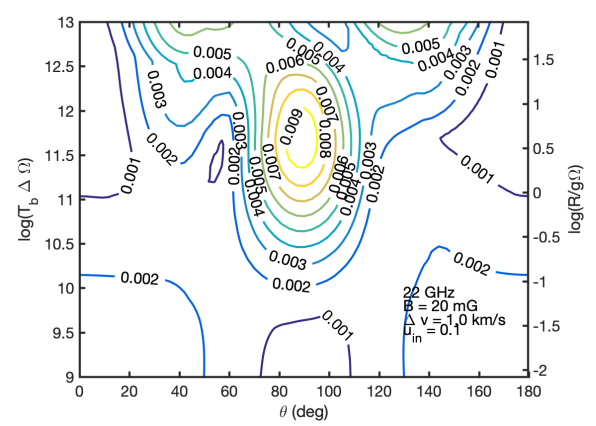

(f)

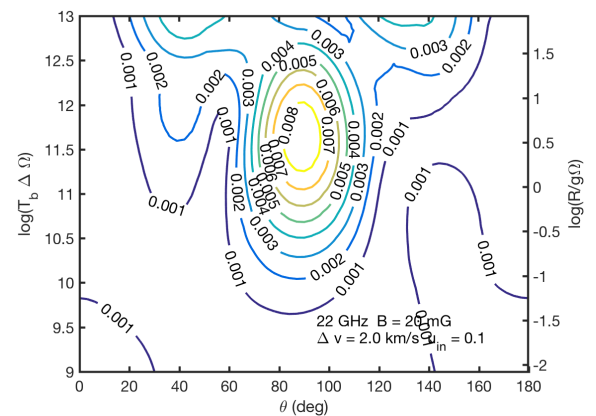

(i)

Fig. A.21. Polarization of a water maser with $10 \%$ polarized seed radiation at $B=20 \mathrm{mG}$. Linear polarization fraction (a,d,g) and angle (b,e,h), and circular polarization fraction (c,f,i). Thermal width used is $v_{\mathrm{th}}=0.6 \mathrm{~km} \mathrm{~s}^{-1}(\mathrm{a}, \mathrm{b}, \mathrm{c}), 1 \mathrm{~km} \mathrm{~s}^{-1}$ (d,e,f), and $2 \mathrm{~km} \mathrm{~s}^{-1}(\mathrm{~g}, \mathrm{~h}, \mathrm{i})$. 
B. Lankhaar and W. Vlemmings: Maser polarization

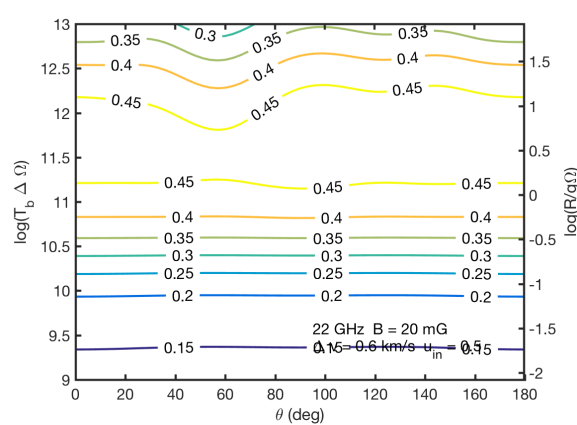

(a)

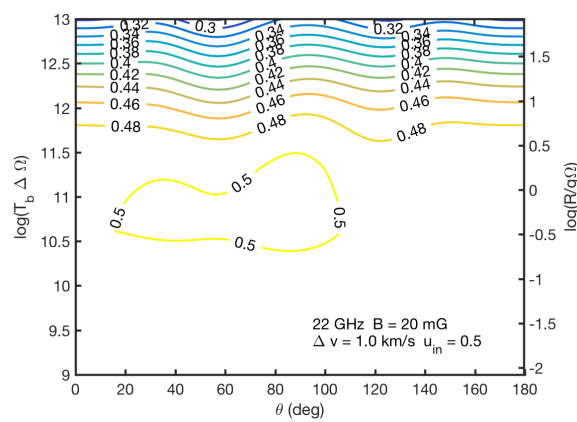

(d)

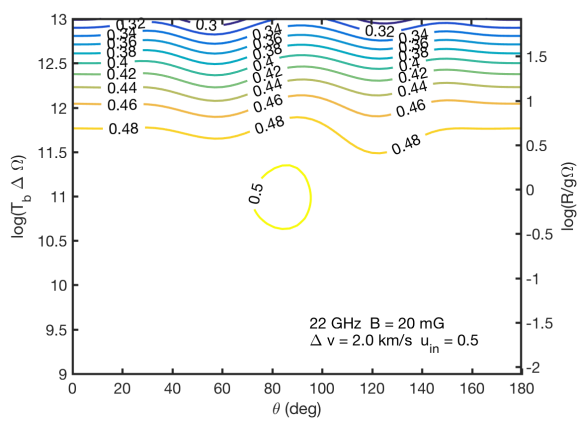

(g)

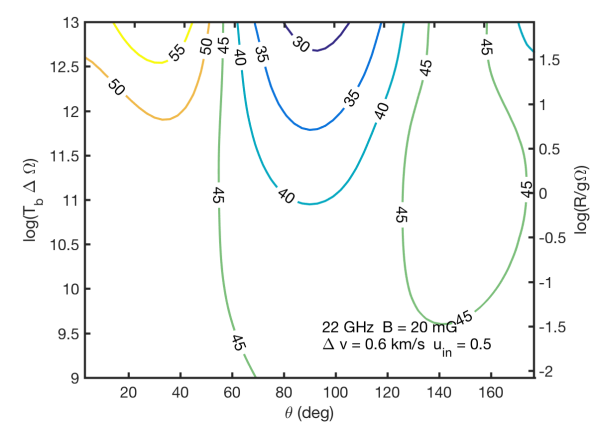

(b)

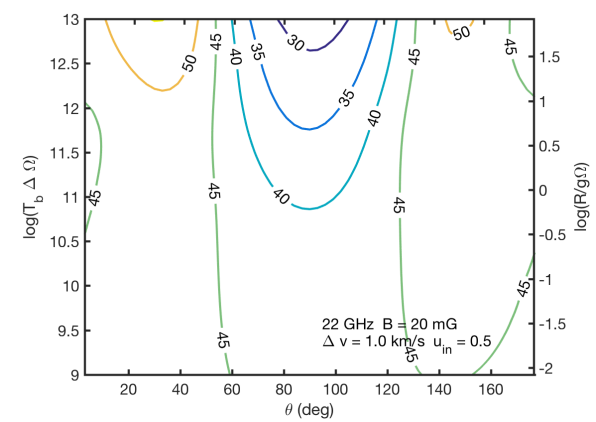

(e)

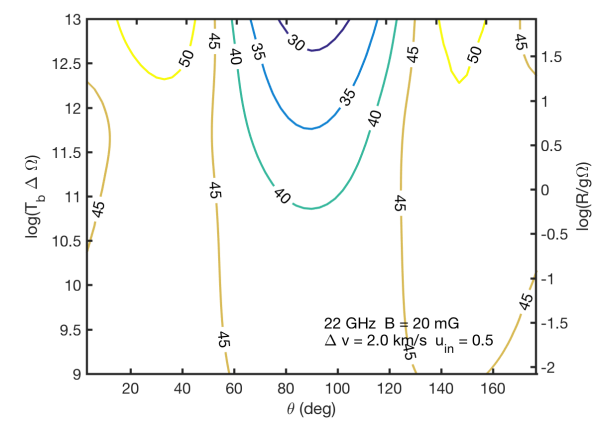

(h)

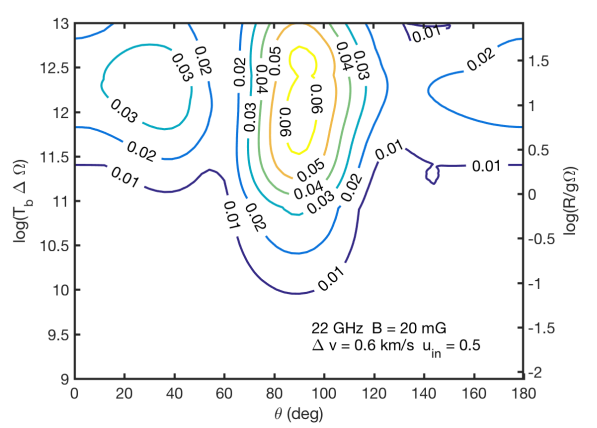

(c)

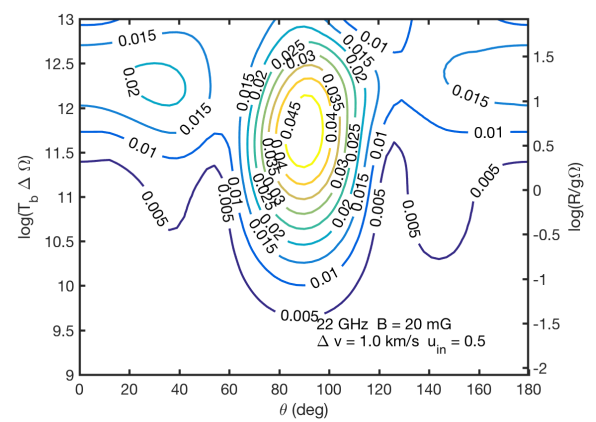

(f)

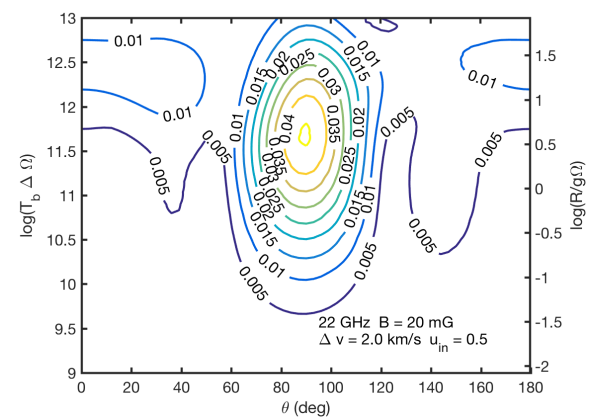

(i)

Fig. A.22. Polarization of a water maser with $50 \%$ polarized seed radiation at $B=20 \mathrm{mG}$. Linear polarization fraction (a,d,g) and angle (b,e,h), and circular polarization fraction (c,f,i). Thermal width used is $v_{\text {th }}=0.6 \mathrm{~km} \mathrm{~s}^{-1}(\mathrm{a}, \mathrm{b}, \mathrm{c}), 1 \mathrm{~km} \mathrm{~s}^{-1}(\mathrm{~d}, \mathrm{e}, \mathrm{f})$, and $2 \mathrm{~km} \mathrm{~s}^{-1}(\mathrm{~g}, \mathrm{~h}, \mathrm{i})$. 